(COO-40406-5)

\title{
INVESTIGATIONS OF NUCLEAR STRUCTURE AND NUCLEAR REACTIONS \\ INDUCED BY COMPLEX PROJECTILES
}

DOE/ER/40406--5

DE93 004602

Progress Report for the Period

September 1, 1991 - August 31, 1992

for

Department of Energy Grant DE-FG02-88ER-40406

D.G. Sarantites - Principal Investigator

Department of Chemistry

Washington University, St. Louis, MO 63130
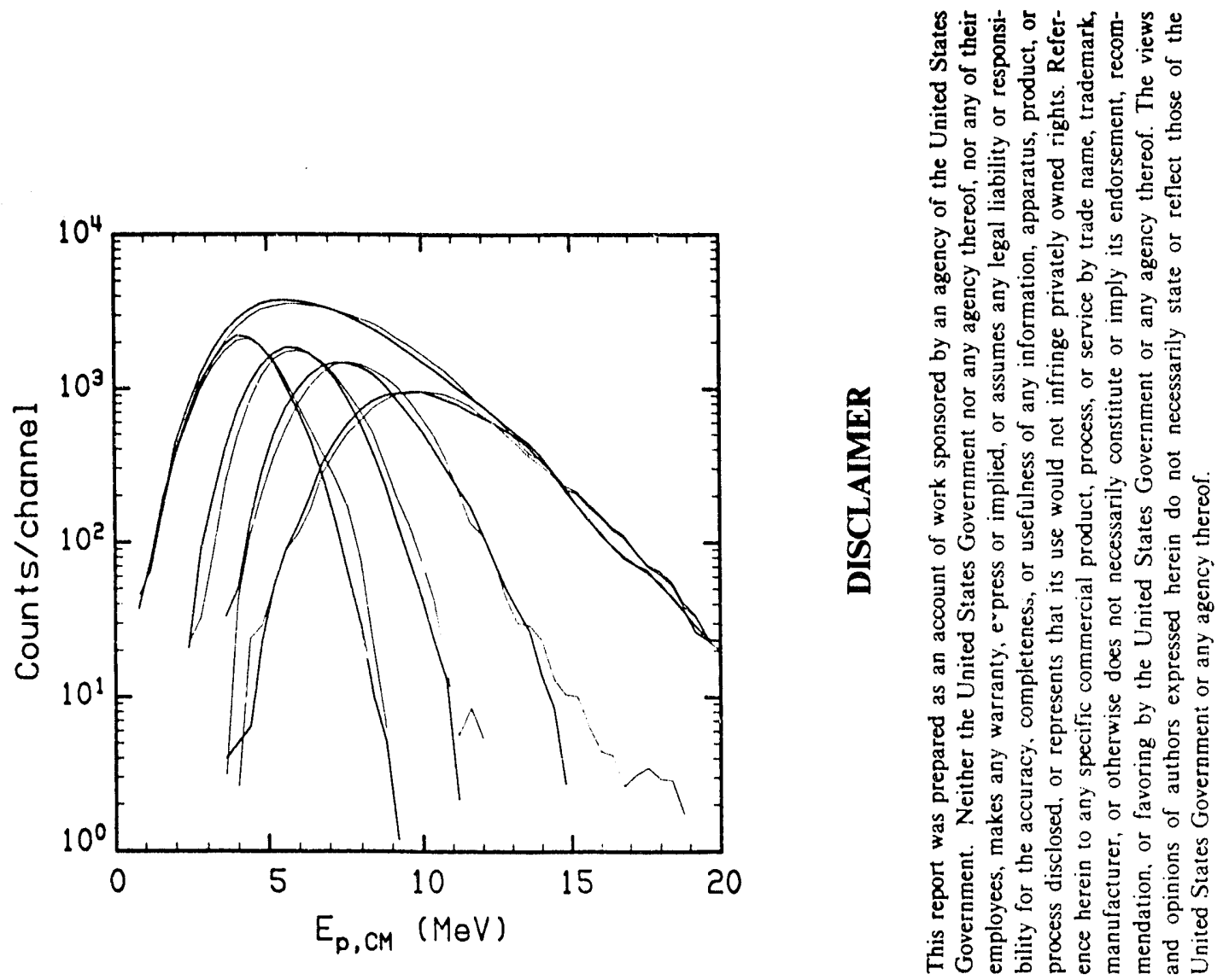


\section{CONTENTS}

I. OVERVIIEW 1

II. SCIENTIFIC RESULTS 6

A. NUCLEAR STRUCTURE STUDIES AT HIGH SPIN 6

1. Search for Hyperdeformation in the $\mathrm{A} \simeq 1.82$ Region. 6

2. Structure of ${ }^{182} \mathrm{Hg}$ and ${ }^{182} \mathrm{Au}$ at High Spin. 8

3. A Highly Deformed Band in ${ }^{136} \mathrm{Pm}$ and the Anomalous $h_{\frac{11}{2}}$ Proton

4. Quiantitative Studies of the Continuum $\gamma$-ray Correlations in ${ }^{170} \mathrm{Hf} \quad 13$

5. Alignment Processes and Shape Variations in ${ }^{184} \mathrm{Pt}$

B. STUdiES AT THE INTERFACE OF STRUCTURE AND REACTIONS 14

1. Population of Entry States in Heavy-Ion Fusion Reactions. 14

2. Nuclear Structure Effects in Proton Evaporation Spectra. 16

3. Nuclear Structure Dependent Entry State Population by Total Spectroscopy. 21

4. Entrance Channel Effects in Fusion near the Barrier. 28

5. Lifetimes of Subbarrier $\alpha$-particles by the Atomic Clock Method 37

6. Large Deformation in $A \sim 170$ Nuclei at High Excitation Energies. $\quad 39$

C. PRODUCTION AND DECAY OF HOT NUCLEI 41

1. The Statistical Model Evaporation Code EVAP. 41

2. Statistical Deuteron and Triton Emission from Highly Excited Compound Nuclei.

3. Heavy-Fragment Emission as a Probe of the Thermal Properties of Highly Excited Compound Nuclei. 47

4. On the Use of IWBC Transmission Coefficients in the Statistical Model: Implicationsin the Evaporation Spectra. $\quad 50$

5. A study in the Transparency in the Optical Model. 53

D. REACTION MECHANISM STUDIES $\quad 59$

1. The Mechanisms for Dissassembly of Excited ${ }^{16} \mathrm{O}$ Projectiles into Four $\alpha$ Particles. 
2. Binary Character of Highly Dissipative ${ }^{136} \mathrm{Xe}+{ }^{207} \mathrm{Bi}$

Collisions at $\mathrm{E}_{\text {lab }} / \mathrm{A}=28.2 \mathrm{MeV}$.

3. A Study of the Multiplicity Dependence of the High Energy Photon Production in a Heavy-Ion Reaction.

III. INSTRUMENTATION AND ANAJYYSIS

A. ADVANCES IN INSTRUMENTATION

1. "Microball" a $4 \pi$ Channel Selecting Device:

An Extension of The Dwarf Ball and Wall System

2. A Novel X-ray Detector

3. A Simple Channel Selecting Detector

4. The Mini Wall

B. COMPUTER FACILITY AND DATA AQUISITION

1. The Nuclear Chemistry Computer Facility: Hardware

2. Aquisition and Analysis Software

IV. SCIENTIFIC AND TECHNICAL PERSONNEL

A. WASHINGTON UNIVERSITY PERSONNEL

B. COLLABORATIONS

V. PUBLICATIONS

A. PAPERS PUBLISHED OR SUBMITTED (1990-1992)

B. INVITED PRESENTATIONS IN MEETINGS (1990-1992)

A. Quantitative Studies of the Continuum Correlations in ${ }^{170} \mathrm{Hf}$

B. Shape Coexistence and Disappearance of Pairing Correlations in ${ }^{82} \mathrm{Sr}$.

C. Alignment Processes and Shape Variations in ${ }^{184} \mathrm{Pt}$.

D. Alpha-particles as Probes of Nuclear Shape in the Rare Earths and Structure Effects on Proton Emission in the Mass-80 Region (Miami).

E. Alpha-particle Emission as a Probe of Nuclear Shapes and Structure Effects in Proton Evaporation Spectra (ORNL).

F. Alpha-particles as Probes of Nuclear Shape and Structure Effects in Proton Evaporation Spectra (Zakopane). 
G. Charged Particles as Probes to Study Entrance Channel Effects in the Composite System ${ }^{164} \mathrm{Yb}^{*}$

H. Observation of Entrance Channel Effects in Compound Nucleus Formation. with high energy $\gamma$-rays.

I. Large Deformation in $\mathrm{A} \sim 170$ Nuclei at High Excitation Energies.

J. Yield Decomposition and Excitation Energy Reconstruction in an Incomplete Fusion Reaction.

K. Determination of the Nuclear Level Density at High Excitation Energy.

L. EVAP: A Monte Carlo Statistical Model Evaporation Code for High Eneryy Compound Nucleus Reactions.

M. Statistical Emission of Deuterons and Tritons from Highly Excited Compound Nuclei.

N. Binary Character of Highly Dissipative ${ }^{136} \mathrm{Xe}+{ }^{207} \mathrm{Bi}$

Collisions at $\mathrm{E}_{l a b} / \mathrm{A}=28.2 \mathrm{MeV}$.

O. The Mechanism for the Disassembly of Excited ${ }^{16} \mathrm{O}$ Projectiles into Four Alpha Particles.

P. Par'icle Multiplicity Dependence of the High Energy Photon Production in a Heavy-Ion Reaction (With Erratum).

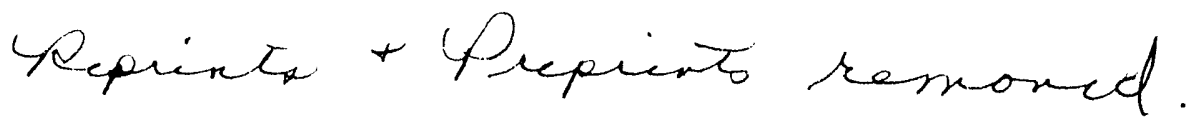




\section{OVERVIEW}

The research program of our group touches five areas of nuclear physics: (1) Nuclear structure studies at high spin; (2) Studies at the interface between structure and reactions; (3) Production and study of hot nuclei; (4) Reaction Mechanism Studies (5) Development and use of novel techniques and instrumentation in the above areas of research.

Significant progress has been made in all the areas of our research program during the past year. The lists of publications and invited talks are given in Chapter $V$. In the past 3 years we published 5 letters, 1 rapid communication and 10 full papers. In addition, 6 publications of invited talks appeared in conference proceedings.

In the following, we highlight our accomplishments and advances in the five areas of our effort.

In the first area, we have undertaken a search for hyperdeformation in the mass 182 region. From a new experiment we have constructed an $\mathrm{E}_{\gamma}-\mathrm{E}_{\gamma}$ matrix mainly for ${ }^{182} \mathrm{Hg}$. We are examining a number of possible candidate transitions that may have the appropriate spacing for the predicted large deformation. An extensive decay scheme for the high spin states in ${ }^{182} \mathrm{Hg}$ has been obtained. Further analysis is in progress to complete this scheme and to assign spins.

We have carried out a search for multiple superdeformed bands in ${ }^{136} \mathrm{Pm}$ and neighboring nuclei. Identical superdeformed bands have been found in the $A=150$ and $A=190$ regions, while searches for multiple $S D$ bands in the $A=130$ region have up to now been overlooked. In our expeiment we have used the Dwarf Ball as a channel selector. We have found superdeformed band in ${ }^{136} \mathrm{Pm}$. Its $\mathrm{J}^{(2)}$ does not vary with rotational frequency, unlike other large deformation bands in neighboring nuclei. This allows us to explain the underlying structure responsible for large deformation in this region. A rapid communication is under preparation.

In the second area we include studies covering the strong interplay between use of reactions and structure. We have continued the analysis of earlier data leading to population of entry states in rate earth systems at energies well above the barrier.

We have further confirmed in new experiments the existence of large shifts in the proton evaporation spectra when different rotational bands are populated by the $\left({ }^{34} \mathrm{~S}\right.$, $2 \mathrm{p} 2 \mathrm{n}$ ) reaction leading to ${ }^{82} \mathrm{Sr}$. A long paper is under preparation summarizing our results in detail.

In a related experiment we employed a new concept of "total spectroscopy" by preparing ${ }^{82} \mathrm{Sr}$ via the $\left({ }^{28} \mathrm{Si}, 4 \mathrm{p}\right)$ reaction, and detecting all 4 protons with the Dwarf Ball and Wall, the $\gamma$-rays with the Spin Spectrometer and the discrete $\gamma$-rays with 18 Compton suppressed Ge detectors. This allows us to determine the excitation energy of the entry states in the residual nucleus without the use of the rather incomplete total $\gamma$-ray energy measurement. Again, we find differences in the proton spectra when the ground or the yrast bands are populated at high spin (see Fig. in the front cover of this report), but the 
magnitude of the differences is smaller compared to the $\left({ }^{34} \mathrm{~S}, 2 \mathrm{p} 2 \mathrm{n}\right)$ reaction. Furthermore, now we can determine the excitation energy distributions that lead to the yrast and ground bands of the ${ }^{82} \mathrm{Sr}$ nucleus at high spin. We find a substantial difference in these distributions. These effects cannot be ascribed to phase space arguments that do not include specific structural information. Thus, it appears that nuclear structure differences at large high excitation are responsible for the observed effects.

We have reinvestigated previous claims for the existence of strong entrance channel effects in the fusion of symmetric and asymmetric projectile-target combinations that are designed to produce compound nuclei at the same excitation energy $(\sim 50 \mathrm{MeV})$. Such effects appeared to violate the Bohr independece hypothesis for the formation and decay of compound nuclei. We found that in the decay of ${ }^{16+4} \mathrm{Yb}^{*}$ (formed by ${ }^{16} \mathrm{O}+{ }^{148} \mathrm{Sm}$ and $\mathrm{Ni}$ $+{ }^{100} \mathrm{Mo}$ ) by $\alpha \mathrm{xn}$ emission, the previously observed differences are due to the breakup of ${ }^{16} \mathrm{O}$ into ${ }^{12} \mathrm{C}+\alpha$ prior to fusion, which is unexpected for such low projectile energies $(87$ $\mathrm{MeV}$ for ${ }^{16} \mathrm{O}$ ). This prevents compound nucleus formation at the same excitation energy and dismisses the differences as due to entrance channel effects. For the xn exit channels we find that the mapping from entrance orbital angular momentum to residue spin, then to $\gamma$-ray multiplicity, and finally to coincidence fold observed with the Spin Spectrometer is responsible for the rather strong previously reported entrance channel effects. This explains all the main features of the data without invoking any unexpected effects. Still there are, however, some aspects that indicate that the detailed fusion processes near the barrier exhibit important differences, that depend on the fusing system.

On a different problem, we know for sometime now that in statistical model calculations a substantial faction of the subbarrier $\alpha$-particles are emitted from low excitations near the yrast line. If this is true, then a fraction of the emitted $\alpha$ 's may have a significantly long lifetime to be measurable by the X-ray atomic clock method. We have measured $\alpha$-particles in coincidence with $\mathrm{K} \mathrm{X}$-rays in the reaction ${ }^{60} \mathrm{Ni}+{ }^{100} \mathrm{Mo} \rightarrow{ }^{160} \mathrm{Yb}^{*}$ at 250 $\mathrm{MeV}$. The majority of the $\mathrm{X}$-rays originate from the daughter Er nuclei following fast $\alpha \times n$ emission and then $\gamma$-decay which produces $K$ vacancies by internal conversion. Observation of the compound nucleus $\mathrm{Yb} \mathrm{X}$-rays in coincidence with the subbarrier $\alpha$ 's indicates that the $\alpha$ emission follows the X-ray emission. Since the latter has a mean life of $4 \times 10^{-18} s$, this provides a clock which shows that part of the $\alpha$-emission is slower than that time scale. We have indeed found a small Yb X-ray peak in coincidence with the low energy $\alpha$ 's and have thus clocked $\alpha$ emission. A fraction of $15-30 \%$ of the subbarrier $\alpha$ particle from high spin states is found to be emitted after the initial compound nucleus $\mathrm{Yb}$ characteristic $\mathrm{X}$-rays. Further analysis of this result is in progress in order to extract the region in $\left(\mathrm{E}^{*}, \mathrm{I}\right)$ plane that corresponds to the slow $\alpha$ emission.

In another experiment we have measured the giant dipole resonance (GDR) in coincidence with fission fragments and with evaporation residues in order to study nuclear shapes at high excitation. We have found evidence for very large deformation from GDR observed in coincidence with fission fragments from the reaction ${ }^{16} \mathrm{O}+{ }^{159} \mathrm{~Tb}$ forming ${ }^{175} \mathrm{Ta}$ at 123.4 
$\mathrm{MeV}$ of excitation. The deformation corresponds to $\beta=0.55$ for $\mathrm{T}=1.8 \mathrm{MeV}$ and it is interpreted to be due to emission from the compound nucleus on the way to fission.

In the third area we addressed the issue of producing hot nuclei in order to study their properties. In a series of two papers we employed an incomplete fusion reaction to fully characterize the excitation energy of hot nuclei. From the energy spectra and light charged particle multiplicities we were able to infer that the level density parameter, $a$, in a Fermi gas description decreases as the excitation energy of the nucleus increases. Whereas the proton and $\alpha$ spectra and multiplicities were both consistent with a reduced level density parameter at high excitation $(\mathrm{T} \sim 3.5 \mathrm{MeV}$ ), the deuteron and triton multiplicities were overpredicted in the simulations employed for extracting the level density parameter. This signified a serious deficiency in our ability to calculate evaporatively deuterons and tritons from highly txcited nuclei.

We have pursued this work further and have shown in a new paper that a description of the deuteron and triton multiplicities and spectra is possible, provided that the corresponding inverse cross sections are reduced over the optical model predictions. For deuterons the required reduction is consistent with experimental deuteron fusion cross sections. This breakdown of the traditional approach is attributed to the large percentage of non-fusion components conlained in the optical model absorption cross section.

We have continued improvements of the statistical evaporation code EVAP. We have extfended the code to include 14 particle decay channels: $\mathrm{n}, \mathrm{p}, \alpha, \mathrm{d}, \mathrm{t},{ }^{3} \mathrm{He},{ }^{6} \mathrm{Li},{ }^{9} \mathrm{Be},{ }^{11} \mathrm{~B}$, ${ }^{12} \mathrm{C},{ }^{14} \mathrm{~N},{ }^{16} \mathrm{O},{ }^{19} \mathrm{~F}$, and ${ }^{20} \mathrm{Ne}$. In these calculations for the first 7 particle modes optical model transmission coefficients are used, and parabolic penetrabilities are used for the remaining emitted particles.

In a separate investigation we have examined the intermediate fragment emission as a probe of the thermal properties of highly excited compound nuclei. Particular attention was given to the low energy part of the IMF spectra which are shown to be much more sensitive to the barriers for hot nuclei compared to the light particle emission.

We have made a systematic study of transmission coefficients obtained via the incoming wave boundary condition (WBC). These were compared with those from the optical model. We found excess cross section for neutron emission predicted by the IWBC which cannot be justified in terms of existing data. The IWBC model was introduced in an attempt to eliminate processes like transparency, shape resonances or peripheral absorption which are expected to be present in the optical model but not in the inverse cross section for emission from excited nuclei. We have been able to study the percentage of transparency contributions in the optical model absorption cross section for alpha particles. The transparency was found to increase with bombarding energy.

In the fourth area number of diverse projects have been carried out. A new method for investigating the mechanism and time scale for the dissassembly of excited projecties has been developed. It relies on the determination of the relative angle distributions of the remnants of the projectile breakup as detected and characterized in a $4 \pi$ detection. 
We have thus studied the breakup of ${ }^{16} \mathrm{O}$ into four $\alpha$-particles and of ${ }^{28} \mathrm{Si}$ into a variety of exit channels such as ${ }^{12} \mathrm{C}+4 \alpha$, or ${ }^{16} \mathrm{O}+3 \alpha$, etc. From detailed simulations of these decay mechanisms we found that the ${ }^{16} \mathrm{O} \rightarrow 4 \alpha$ decay is exclusively sequential, while ${ }^{28} \mathrm{Si} \rightarrow{ }^{12} \mathrm{C}+4 \alpha$ is a mixture of sequential decay and fission. Information about the time scales has also been obtained.

We have studied dissipative collisions of ${ }^{124} \mathrm{Xe}$ with ${ }^{207} \mathrm{Bi}$ at $28.2 \mathrm{MeV} /$ nucleon, by combining the Dwarf Ball and Wall with the $\mathrm{U}$. of Rochester $4 \pi$ neutron multiplicity meter. We found that there is a strong correlation between the neutron and charged particle multiplicities. There is, however, an important delay in the charge particle multiplicity, indicating that a low excitations neutron emission dominates, whereas for high excitation both particles and neutrons are emitted in a correlated way. Furthermore, for any degree of dissipation, the velocity distributions of charged particles are found to be characteristic of sequential emission following binary collisions. The average correlation between multiplicities of neutrons and charged particles can be understood with the same reaction scenario. The energy dissipation appears to be approximately only one-half of the available entrance channel kinetic energy.

In the last area of instrumentation and analysis we have made substantial progress. We have improved the Dwarf Ball detector system by building 18 2-mm thick CsI(T $\ell)$ detectors that can be used in front of Ge detectors to minimize absorption. We have now designed, constructed and used a set of new discriminators (112 channels) capable of discriminating in width and amplitude. This allow $\not s$ us to eliminate essentially all the electron pulses from the target and thus lower the thresholds significantly.

A new set of multihit TDC have been acquired ordered and have been used in several experiments with the DBW and the new Miniwall. The latter is a 110 detector hodoscope compatible with the MSU Miniball which was designed and constructed at Washington University by L. Sobotka. The new discriminators and the multihit Lecroy TDC's were our contribution to this project.

We have made progress in the design of a new $4 \pi$ device the "Microball", the next generation of the Dwarf Ball. It is based on $96 \mathrm{CsI}(\mathrm{T} \ell)$ scintillators with PIN diode readout. Tests of prototype detectors are reported. The devicent has (a) a mass of $1 / 8$ of that of the Dwarf Ball, (b) an increasing forward segmentation, (c) good channel identification capability in $4 \pi$, (d) low thresholds for all light charged-particle channels, and (e) it can handle all reasonable event rates for spectroschpic studies.

We have designed and constructed a low cost channel selecting device, capable of enhancing the xn channels by tagging and removing charged-particle channels and fission. It consists of a scintillator tube made of four fast-plastic segments with light collection at one end. It has high rate capability, but it cannot identify the particle channels. The device was utilized very succesfully in a spectroscopy experiment for the high spin structure of ${ }^{182} \mathrm{Hg}$. 
We have designed and purchased a novel X-ray detector for timing nuclear processes such as $\alpha$ emission and fission by the atomic clock method.

We have made improvements in our computer system. We have also obtained the necessary hardware and converted our computer system to a local area cluster, independent of the department of chemistry network. We have developed new data analysis graphics software for DEC windows. We have also acquired a second $\mathrm{PC} / 486$ computer to run commercial graphics packages that allow us to prepare better quality and faster graphics and to acquire data via the CAMAC interface or as a pulse-height analyser.

We have developped a new data compression package that permits one- and twodimensional histograms to be stored on disk with high compression. This increases the I/O speed and saves typically another factor of 3 over our previous compression scheme. 


\section{SCIENTIFIC RESULTS}

\section{IIA. NUCLEAR STRUCTURE STUDIES AT HIGH SPIN}

IIA1. Search for Hyperdeformation in the Mass $A \simeq 182$ Region - P-F. Hua, D.G. Sarantites, J. Barreto, A. Kirov L. Sobotka [WU], C. Baktash, I.Y. Lee, J.D. Garrett, N.R. Johnson, F.K. McGowan, D.Cullen [ORNL],

Following the initial discovery of discrete superdeformed bands in ${ }^{132} \mathrm{Ce}$ and ${ }^{152} \mathrm{Dy}[1]$ this area of $\gamma$-ray spectroscopy has seen a great deal of activity by many groups worldwide. In the last few years however, almost all the superdeformed (SD) spectroscopy groups have concentrated on the $\mathrm{A}=150$ and new $\mathrm{A}=190$ regions. The excitement in these latter regions has been intense, particularly since non-yrast SD bands were observed in individual nuclei, which has led to the remarkable discovery of "identical" SD bands in neighboring nuclei.[2]

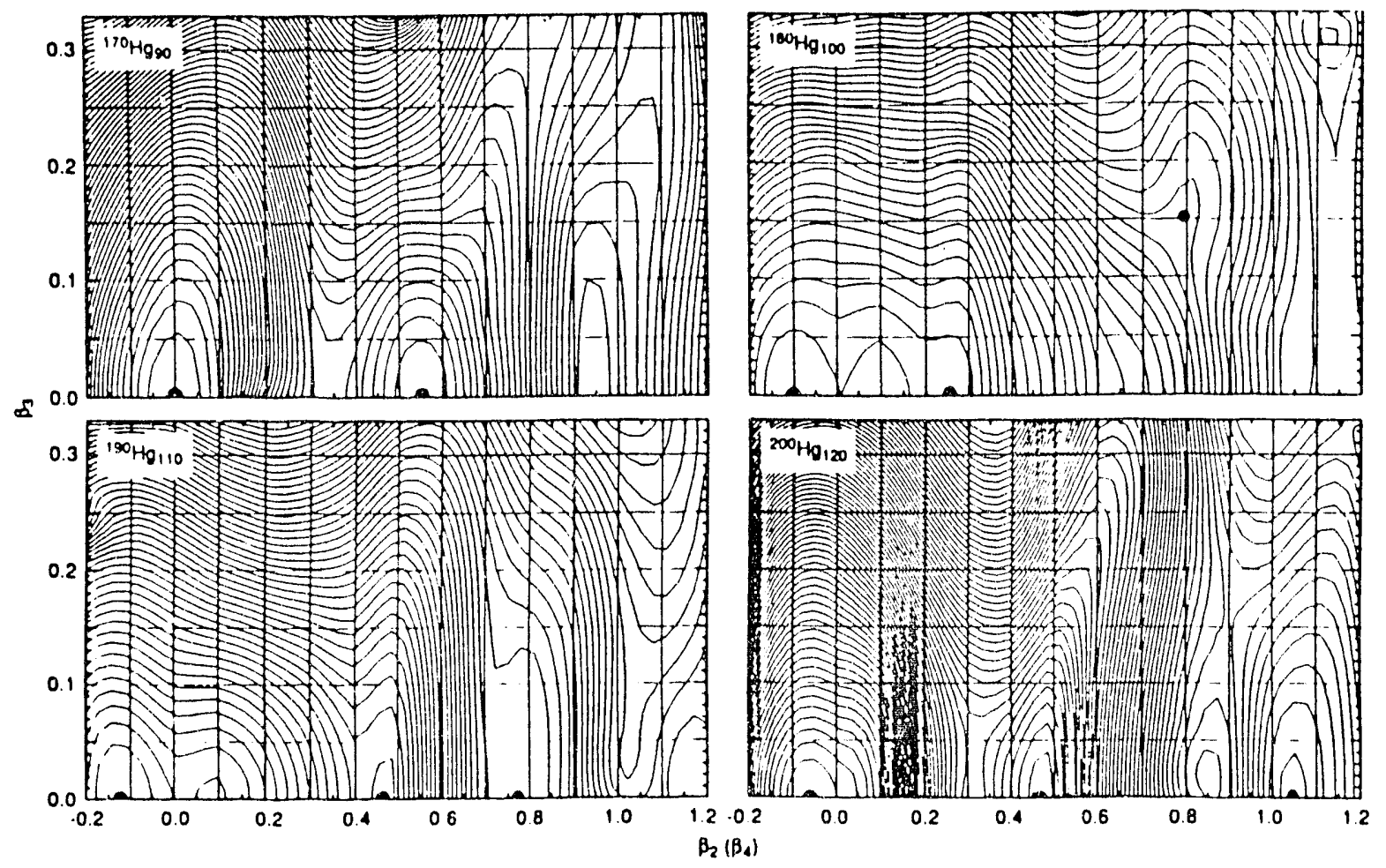

Figure IIA 1.1. Calculated potential energy surfaces at $h \omega=0$, [3], for ${ }^{170} \mathrm{Hg}_{90},{ }^{180} \mathrm{IIg}_{100},{ }^{190} \mathrm{Ig}_{110}$, and ${ }^{200} \mathrm{Hg}_{120}$ as a function of $\beta_{2}$ and $\beta_{3}$. The loci of the minima are shown by the solid dots.

An even more exciting possibility is the observation of even larger deformations with $\beta=0.8-1.0$ ( $\sim 3: 1$ axes ratio). Such large deformation, termed "hyperdeformation", has been predicted to exist in a number of regions at different spins and excitation energies. Usually the hyperdeformation is predicted for very high spins $\sim \$ 0 \hbar$, where the fission barrier vanishes. Very recently, state of the art calculations by Nazarewic have predicted 
systematically hyperdeformation at low excitations in the light $\mathrm{Hg}$ isotopes. [3] The potential energy surfaces in the $\beta_{2}$ vs. $\beta_{3}$ plane at zero rotational frequency are shown in Fig. IIA 1.1, and the excitation energy of the minimum is shown as a function of neutron number in Fig. IIA1.2. A minimum at $\beta_{2} \sim 0.8$ is predicted in ${ }^{180} \mathrm{Hg}$ at $\sim 6.5 \mathrm{MeV}$ of excitation.

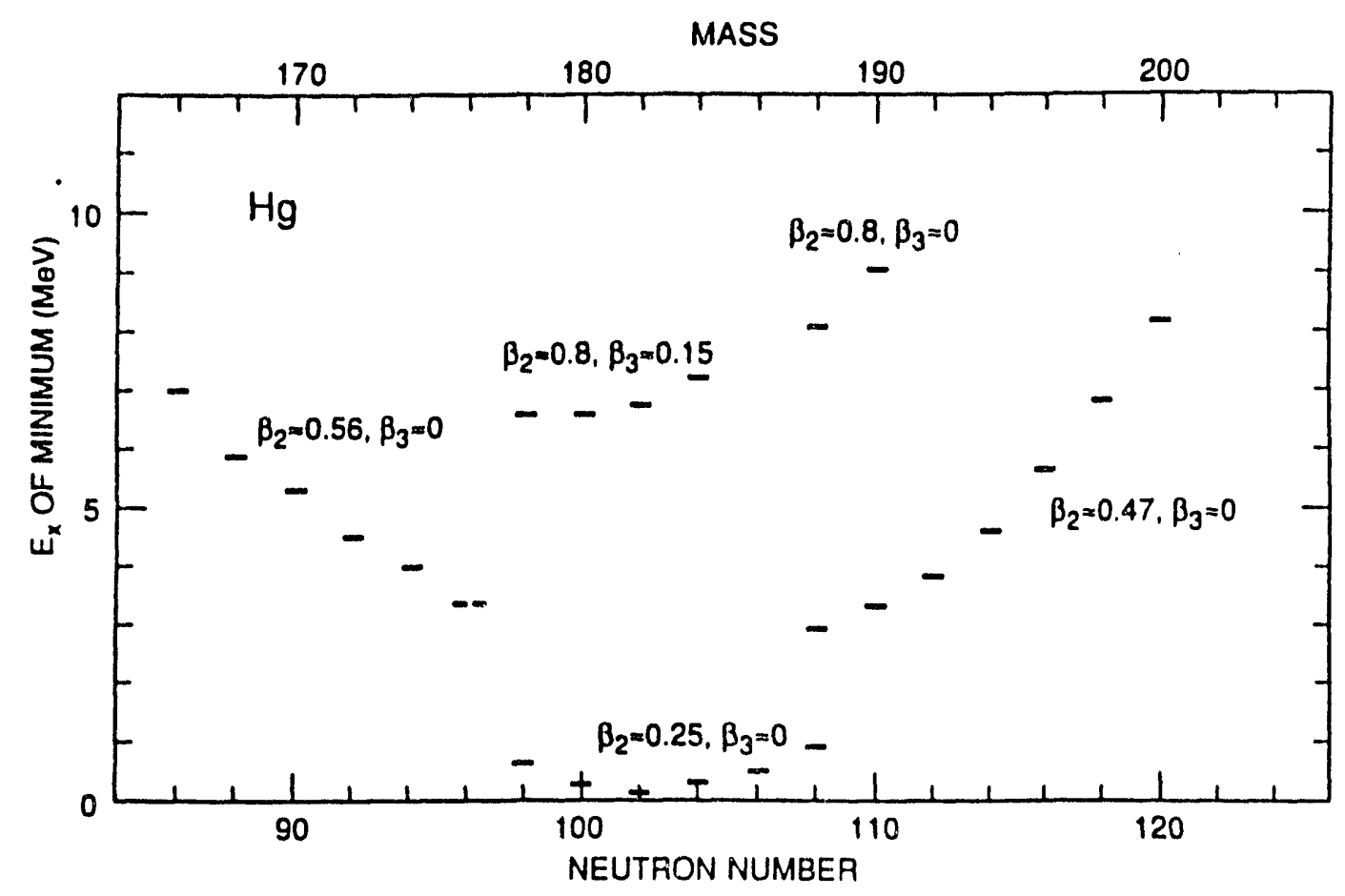

Figure IIA1.2 Calculated excitation energies [3] of the second minima in the potential energy surface for the $\mathrm{Hg}$ isotopes. For each set the values of $\beta_{2}$ and $\beta_{4}$ are shown.

Such a shape at low excitation would correspond to spin $\sim 30-35$ and may survive fission. Based on this prediction, we derided to undertake a search for a large deformation band structure in ${ }^{182} \mathrm{Hg}$. For this purpose we chose the reaction ${ }^{154} \mathrm{Gd}\left({ }^{32} \mathrm{~S}, 4 \mathrm{n}\right){ }^{182} \mathrm{Hg}$.

Statistical model estimates of the cross sectins indicated only $\sim 30 \mathrm{mb}$ for the $4 \mathrm{n}$ channel, $200 \mathrm{mb}$ of fission and the remaining $150 \mathrm{mb}$ in charged particle channels. Based on this we designed a special particle counter that would allow us to tag and veto the charged-particle and fission channels. This counter was made from fast plastic scintillator and had the shape of a tube segmented in 4 . The performance of this detector is described in the Section III. The experiment was performed at the HHIRF with the compact Ge array. A total of 12 Compton suppressed Ge detectors were used in the Compact Ball configuration. Four additional anti-Compton shields and four inserted $\mathrm{BaF}_{2}$ detectors served as a multiplicity filter to select high multiplicity events. The charged-particle channels to to a considerable extent the fission events were identified. 
In the above arrangement it was possible to construct a high multiplicity $\mathrm{E}_{\gamma}-\mathrm{E}_{\gamma}$ matrix from which the charged-particle channels and fission were removed. This matrix was then examined for sequences of $\gamma$-rays corresponding to the expected energies. Although we found some candidates for these iransitions (only 4-5) transitions, we could not unambiguously establish the rotational character of these structures. We are still analyzing the data, but at present we can place an upper limit at the $1 \%$ level for the population of a hyperdeformed structure.

This work was done in collaboration with the ORNL group, while the data analysis was done primarily by P.F. Hua and the P.I.

[1] C.G. Anderson et al., Phys. Scripta 24, 266 (1981).

[2] J. Dudek and W. Nazarewicz, Phys. Rev., C31, 298 (1985).

[3] W. Nazarewicz (private communication and to be published in "Proceedings of the International Conference on Nuclear Structure at High Angular Momentum", Ottawa, May, 1992).

IIA2. Structure of ${ }^{182} \mathrm{Hg}$ and ${ }^{182} \mathrm{Au}$ at High Spin. P-F. Hua, D.G. Sarantites, J. Barreto, A. Kirov, L. Sobotka [WU]. C. Baktash, I.Y. Lci, J.D. Garrett, N.R. Johnson, F.K. McGowan, D.Cullen [ORNL],

The detailed structure of the ${ }^{182} \mathrm{Hg}$ is in itself interesting becanse in the $\mathrm{N}=100$ region the normal and large deformation bands the energies of the potential energy minima are lowest. Yet due to the proximity of the $Z=82$ shell closure, the ground state has a spherical shell model structure (see Figs. IIA1.1 and 2 in Sect. IIA.1).

Very little is known at this time about the structure in ${ }^{182} \mathrm{Hg}$ [1]. A preliminary analysis of the data from the $E_{\gamma}-E_{\gamma}$ matrix provided us with a detailed decay scheme shown in Fig. IIA2.1. Five decay sequences have been identified thus far, but only the four shown in Fig. IIA2.1 have been directly connected to the yrast band. Only levels up to $12^{+}$in the yrast band were previously known [1].

Further analysis is in progress to establish the complete sequences, and when possible determine the level spins sequences from angular correlation information.

Similarly, as by product of this work the structure of ${ }^{182} \mathrm{Au}$ will be obtained. Here the particle gating is used to select only events were only one charged particle was emitted. The matrix can then be cleaned from the 2 particle channel by subtracting a normalized matrix from events in which 2 sectors of the plastic detector fired. The $\left({ }^{32} \mathrm{~S}, \mathrm{xn}\right)$ channels are removed from in these matrices.

This work was done in collaboration with the ORNL group, while the data analysis for ${ }^{182 / \text { was }}$ wone by P.F. Hua and the P.I. This work is part of the PhD thesis of Peng-Fei Hua.

[1] W.C. Ma et al., Phys. Lett. 139B, 276 (1984). 


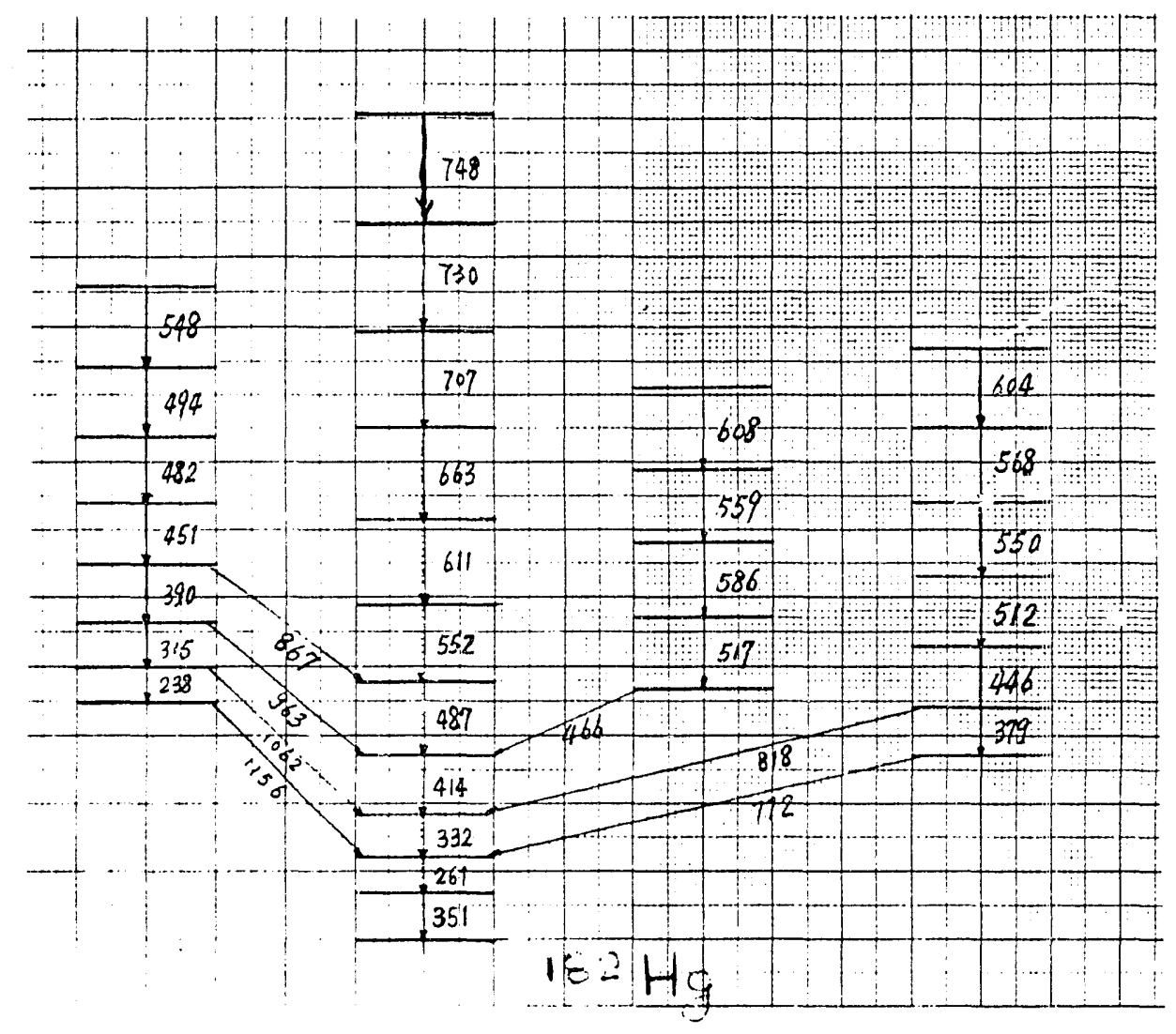

Figure IIA2.1. Preliminary decay scheme for ${ }^{1 \diamond 2} \mathrm{Hg}$ from this experiment.

IIA.3. A Highly Deformed Band in ${ }^{136} \mathrm{Pm}$ and the Anomalous $h_{\frac{11}{2}}$ Proton Crossing in the A 135 Superdeformed Region-- M.A Riley,T. Petters, J. Shick, [FSU], R.J. Charity, D.G. Sarantites, L.L. Wittmer [WU], C. Baktash, W. Gao, J.D. Garrett, M.L. Halbert, D.C. Hensley, I.Y. Lee [ORNL], and J. Simpson [Daresbury].

The dynamical moments of inertia $\left(J^{(2)}\right)$ of superdeformed (SD) bands in the A 150 $\mathrm{Gd}-\mathrm{Dy}$ and $\mathrm{A} \sim 190 \mathrm{Hg}-\mathrm{Pb}$ regions are fairly well reproduced by standard cranking calculations $[1,2]$. However, for the superdeformed or highly deformed A $135 \mathrm{Ce}-\mathrm{Nd}-\mathrm{Sm}$ region, this is not true at all, and has presented a theoretical puzzle for some time. Several theoretical suggestions in terms of quenched proton pairing [3] and a strong residual proton-neutron interaction $[4,5]$ have been proposed.

Up to the present time in the $A \sim 135$ region highly deformed bands have been observed almost exclusively in even- $Z$ nuclei, i.e. ${ }^{131,132} \mathrm{Ce}[6,7,8],{ }^{133,135,136,137} \mathrm{Nd}[9-12],{ }^{135,137} \mathrm{Sm}$ $[3,14,19,16,15]$ and ${ }^{139,141} \mathrm{Gd}[20,21]$. Only in ${ }^{130} \mathrm{La}$ [22] has a highly deformed band been observed in an odd- $Z$ nucleus. Unfortunately this band, since it is only observed at very high frequency ( $\hbar \omega>0.4 \mathrm{MeV}$ ), is not able to contribute significantly to the understanding of the anomalous $J^{(2)}$ dependence on rotational frequency, which centers around the behavior of the lowest $h_{\frac{11}{2}}$ proton levels near $\hbar \omega=0.4 \mathrm{MeV}[3,4,5,13]$. The aim of the 
present work was to observe a highly deformed band in the $N=75$ odd- $Z$ nucleus ${ }^{136} \mathrm{Pm}$ which lies in the middle of this region.

In July/Augnst last year an experiment was performed at the Holified Heavy Ion Research Facility at Oak Ridge to search for this superdeformed band. The reaction used was ${ }^{34} \mathrm{~S}+{ }^{106} \mathrm{Pd}$ at a hombarding energy of $165 \mathrm{MeV}$. Because charged particle emission competes on an equal footing with neutron emission in this mass region, the Dwarf Ball was used to select between the pxn, 2pxn, $\alpha \times n$, p $\alpha x n$, etc., channels. High resolution $\gamma$-ray information was obtained using 18 Compton suppressed Ge detectors and fold and total energy information obtained from the Spin Spectrometer. A total of $280 \times 10^{6} \gamma-\gamma-$ charged particle coincidences were recorded. Additional statistics were accumulated in another experiment carried out at FSU using 9 Compton suppressed Ge detectors and a 28 element BGO sum energy/multiplicity ball.

Figure IIA3.1 shows a summed coincidence spectrum for the highly deformed Pm band observed in both experiments. The band is assigned to ${ }^{136} \mathrm{Pm}$ from observed coincidence relationships with low spin yrast transitions in ${ }^{136} \mathrm{Pm}[25]$ and also excitation function information from the two experiments. The measured intensity of the band is $\sim 5-8 \%$ of the ${ }^{136} \mathrm{Pm}$ channel and closely follows the intensity pattern with increasing spin observed for other highly deformed bands in this region, see inset in Fig. IIA3.1. At the present time no firm connections linking the highly deformed band to the normal deformed levels have been established.

In odd- $N$ nuclei in the $A \sim 135$ region the highly deformed structures occur by having a single neutron occupying the [660] $\frac{1}{2} \mathrm{i}_{\frac{13}{2}}$ orbital [13]. In ${ }^{136} \mathrm{Pm}$ the odd-proton is expected to occupy the favored $h_{\frac{11}{2}}$ signature $\left(\alpha=-\frac{1}{2}\right)$ level. Thus, the yrast highly deformed band is expected to have even spin values with the configuration $\pi\left(h_{\frac{11}{2}}\right)^{1} \otimes \nu\left(i_{\frac{13}{2}}\right)^{1}$. TRS calculations predict a deformation of $\beta_{2} \sim 0.33, \gamma \sim 0$ for the highly deformed minimum compared to $\beta_{2} \sim 0.20, \gamma \sim-20^{\circ}$ values for the normal deformed low spin yrast structures [26]. Since it is the $i_{\frac{13}{2}}$ neutron which plays the dominant deformation driving role, the deformations for all the $\mathrm{N}=75$ isotones are expected to be rather similar. This expectation is supported by the measured quadrupole moments, see Ref. [16].

As mentioned above, the "anomalous" $J^{(2)}$ behavior in this region centers around the $h_{\frac{11}{2}}$ proton alignment expected to take place around $\hbar \omega \sim 0.4 \mathrm{MeV}$. If our configuration assignment to the ${ }^{136} \mathrm{Pm}$ band is correct then this troublesome crossing should be blocked. The observed $J^{(2)}$ for ${ }^{136} \mathrm{Pm}$ shown in Fig. IIA3.2 supports this interpretation, where for $\hbar \omega \sim 0.3-0.5 \mathrm{MeV}$ the band is falling to a near constant value. On the other hand the even$\mathrm{Z} \mathrm{N}=75$ isotones ${ }^{135} \mathrm{Nd},{ }^{137} \mathrm{Sm}$ and ${ }^{139} \mathrm{Gd}$ display significant increases in their $J^{(2)}$ behavior throughout this frequency range, see Fig. IIA3.2. These data indicate that the $h_{\frac{11}{2}}$ proton crossing is indeed taking place in the even- $\mathrm{N}=75$ nuclei (and presumably other even- $\mathrm{Z}$ highly deformed nuclei in this region) but with a much stronger interaction strength than predicted by standard cranking calculations. Thus we can rule out the suggestion that proton pairing has been quenched a very low frequency $(\hbar \omega<0.2 \mathrm{MeV})$ in these highly 


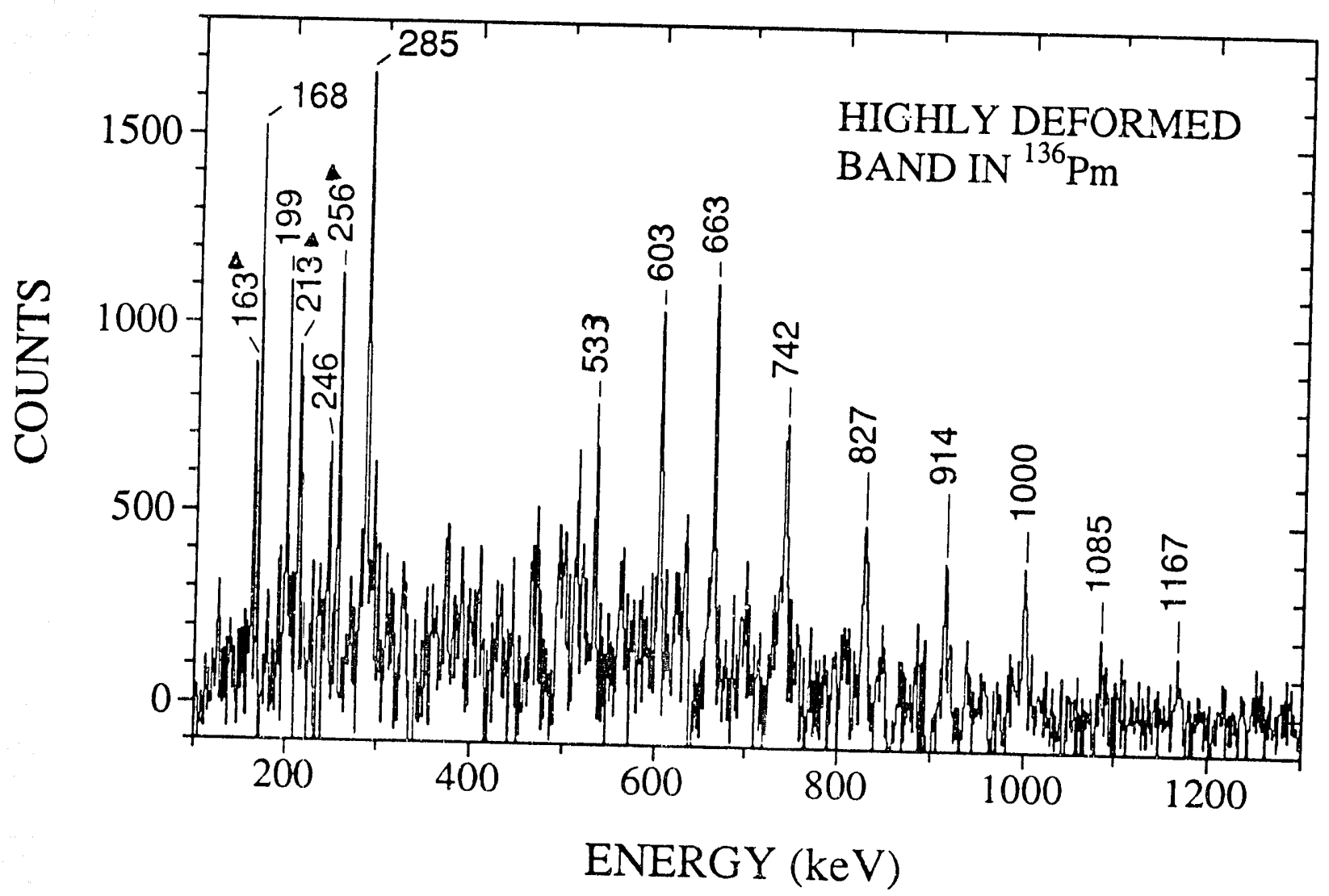

Figure IIA3.1. Summed coincidence spectrum for the highly deformed band observed in ${ }^{136} \mathrm{Pm}$. Energies are given to the nearest $\mathrm{keV}$. Known contaminant lines from ${ }^{131} \mathrm{Ce},{ }^{136} \mathrm{Sm}$ are indicated by a filled triangle.

deformed bands [3]. Our results would support the proposal of Wyss and Johnson [4] for a strong residual proton-neutron interaction between the $\pi h_{\frac{11}{2}}$ and $\nu i_{\frac{13}{2}}$ orbitals which has the effect of shifting the $\pi h_{\frac{11}{2}}$ crossing to slightly higher frequency and causing a much stronger mixing between the crossing bands in the even- $\mathrm{Z} N=75$ nuclei. However we would suggest that this may not be the only possible explanation. We would also suggest that the sharp increases in $J^{(2)}$ observed at very low frequency in the highly deformed bands (see Fig. IIA3.2) are not due to the paired to unpaired phase transition but simply reflect the mixing with other levels of similar $(\pi, \alpha)$ which help initiate the decay out of the highly deformed bands. 


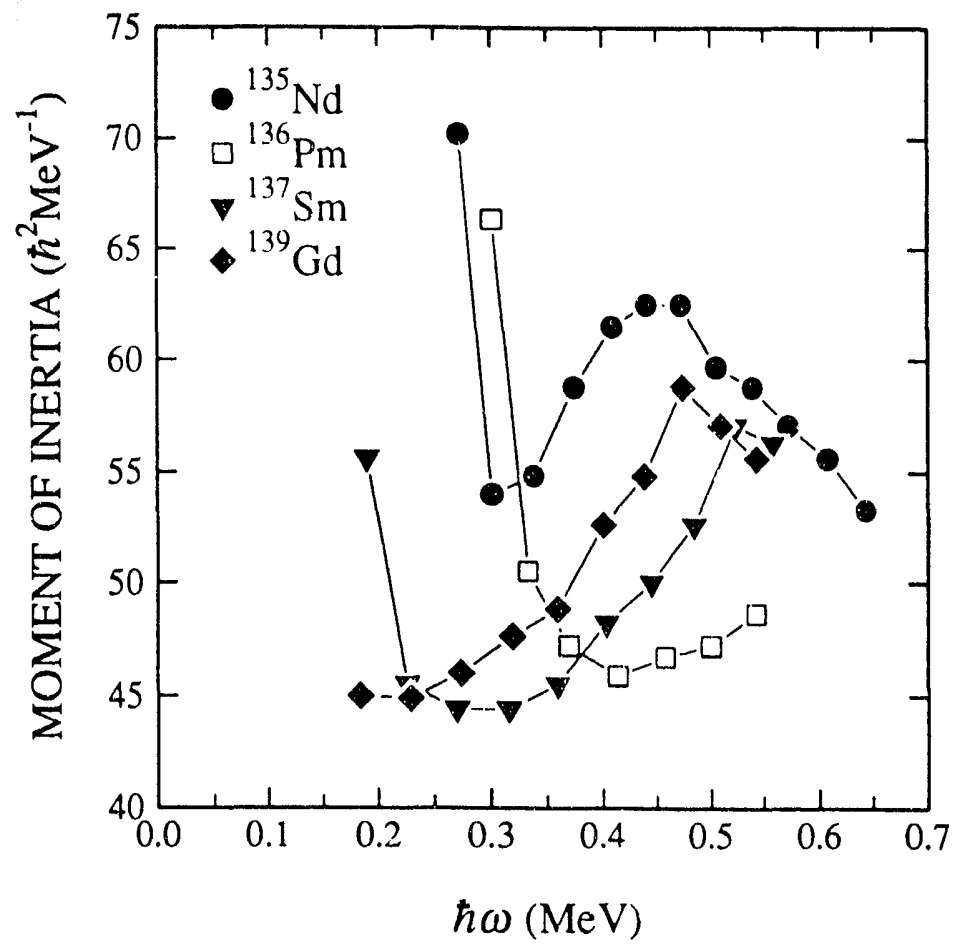

Figure IIA3.2. Plot of the dynamical moment of inertia $J^{(2)}$ against rotational frequency $\hbar \omega$ for highly deformed bands observed in $\mathrm{N}=75$ isotones. Note the rise in $J^{(2)}$ above $\hbar \omega=0.3 \mathrm{MeV}$ for the $\mathrm{Nd}, \mathrm{Sm}$ and Gd bands compared to the downsloping or'nearly constant value for Pm.

This work was done in collaboration with Mark Riley's group and the ORNL group. The data analysis was done by the FSU group.

[1] W. Nazarewicz, R. Wyss, and A. Johnson, Nucl. Phys. A503, 285 (1989).

[2] M.A. Riley et al., Nucl. Phys. A512, 178 (1990).

[3] P.H. Regan et al., Phys. Rev. C42, R1805 (1990).

[4] R. Wyss and A. Johnson, Proc. of the Int. Conf. on High Spin Physics, ed. J.X. Saladin, R.A. Sorensen and C.M. Vincent, (World Scientific, Singapore). 1991, p. 123.

[5] R. Wyss, Symposium on Reflections and Directons in Low Energy Heavy-Ion Physics, Joint Institute for Heavy-Ion Research, Oak Ridge, Oct. 1991, in press.

[6] S J. Nolan et al., J. Phys. G: Nucl. Phys. 11, L17 (1985).

[7] A.J. Kirwan et al., Phys. Rev. Lett. 58, 467 (1987).

[8] Y.X. Luo et al., Z. Phys. A329, 125 (1988).

[9] R. Wadsworth et al., J. Phys. G 13, 207 (1987).

[10] E.M. Beck et al., Phys. Rev. Lett. 58, 2182 (1987).

[11] E.M. Beck et al., Phys. Lett. B195, 531 (1987).

[12] R. Wadsworth et al., Z. Phys. A333, 409 (1989).

[13] R. Wyss et al., Phys. Lett. B215, 211 (1988).

[14] S.M. Mullins et al., J. Phys. G: Nucl. Phys. 13, L201 (1987).

[15] E.S. Paul et al., Phys. Rev. Lett. 61, 42 (1988).

[16] P.H. Regan et al., J. Phys. G: Nucl. Phys. 18, 847 (1992).

[19] R. Ma et al., Phys. Rev. C40, 156 (1989).

[20] R. Ma et al., J. Phys. G: Nucl. Phys. 16, 1233 (1990).

[21] S.M. Mullins et al., private communication.

[22] M. Godfrey et al., J. Phys. G 15, 163 (1989). 
[23] M. Jääskeläinen et al., Nucl. Instr. and Meth. 204, 385 (1983).

[24] D.W. Stracener et al., Nucl. Instr. and Meth. A294, 485 (1990).

[25] C.W. Beausang et al., Phys. Rev. C36, 1810 (1987).

[26] R. Wyss, private communication.

IIA4. Quantitative Studies of the Continuum $\gamma$-ray Correlations in ${ }^{170} \mathrm{Hf}-$ I.Y. Lee, C. Baktash, J.R. Beene, M.L. Halbert, N.R. Johnson, F.K. MícGowan, [ORNL], M.A. Riley [ORNL and JIHHR], and D.G. Sarantites [WU].

This work is a complete study which was recently published in Phys. Rev. C (See Appendix A). Bellow is the abstract from this work.

\begin{abstract}
We have studied the two-dimensional energy correlation of the continuum $\gamma$ rays in ${ }^{170} \mathrm{Hf}$ produced in a heavy-ion fusion reaction. The $\gamma$ rays were measured with an array of Comptonsuppressed Ge detectors and the Spin Spectrometer. A quantitative analysis has been carried out on the properties of the peaks and dips in the correlation spectra. The results indicate the existence of at least two widths in the $\gamma$-ray energy distribution: a narrow width of about $20 \mathrm{keV}$, most likely originating from decay along different discrete bands near the yrast line, and a wide width of about $200 \mathrm{keV}$ from mixed states at higher excitation energy. Theoretical calculations including the effect of level mixing fluctuations in the rotational frequency and moment of inertia give results in general agreement with experiment.
\end{abstract}

This work was done in collaboration with the ORNL group, while the data analysis and interpretation was done primarily by I.Y. Lee of ORNL.

\title{
IrA5. Alignment Processes and Shape Variations in ${ }^{184} \mathrm{Pt}-$ M.P. Carpenter et al.
}

This work is a completed study published in Nucl. Phys. (See Appendix C.) Bellow is the abstract from that work which was done by M. Carperter at UT.

Abstract: High-spin states in the transitional nucleus ${ }^{184} \mathrm{Pt}$ were populated via the reactions ${ }^{154} \mathrm{Sm}\left({ }^{34} \mathrm{~S}, 4 \mathrm{n}\right){ }^{184} \mathrm{Pt}$ and $\left.{ }^{172} \mathrm{Yb}\left({ }^{16} \mathrm{O}, 4 n\right)\right)^{184} \mathrm{Pt}$. The yrast band was excited up to $I=28 \hbar$ and six new side bands built on both neutron and proton quasiparticle configurations were observed. Shell correction-type calculations indicate variations of the nuclear shape in different bands, especially as a result of band crossings due to the process of angular momentum alignment. Comparison of the band characteristics are made between ${ }^{184} \mathrm{Pt}$ and eight adjacent nuclei. The pattern of band crossings in these nine nuclei is considered from the viewpoint of blocking comparisons and of theoretical calculations. The competition between low-frequency $\nu i_{13 / 2}$ and $\pi h_{9 / 2}$ band crossings is discussed. 
IIB1. Population of Entry States in Heavy-Ion Fusion Reactions. - N.G. Nicolis, K. Honkanen*, D.G. Sarantites, T.M. Semkow, M. Rajagopalan, M. Jääskeläinen, R. Woodward, F.A. Dilmanian [WU]. J.R. Beene, D.C. Hensley, J.H. Barker, M.L. Halbert, S.P. Sorensen and W.T. Milner [ORNL].

The transfer of orbital angular momentum into intrinsic spin of the reaction products plays an important role in reaction mechanisms. For fusion reactions spin distributions and associated excitation energy distributions in the evaporation residues can be deduced from measurements of the population of the entry states, which is accomplished with the Spin Spectrometer.

A few years ago we completed a set of measurements addressing the careful measurement of the population of the entry states in several systems including $136 \mathrm{MeV}^{20} \mathrm{Ne}+$ ${ }^{146} \mathrm{Nd}$ and $224 \mathrm{MeV}{ }^{50} \mathrm{Ti}+{ }^{130} \mathrm{Te}$. The cross section and the entry state population for the major exit (HI, xn) and (HI, $\alpha x n)$ channels were measured.

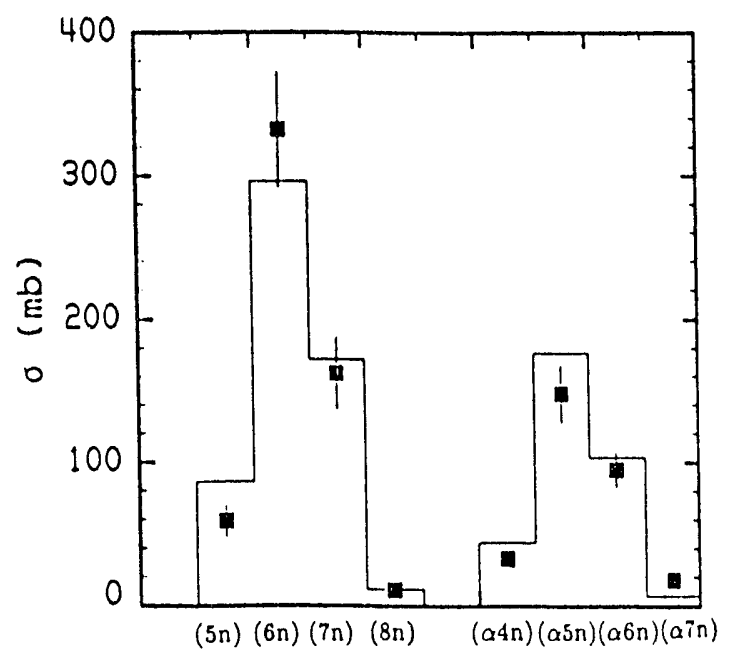

Figure IIB1.1. Comparison of calculated (histograms) and experimental (points) channel cross sections for the reaction $136 \mathrm{MeV}{ }^{20} \mathrm{Ne}+{ }^{146} \mathrm{Nd}$.

For the statistical model description of these data, we employ the evaporation code EVAP, described elsewhere in this report. Since EVAP differs in accuracy and features from the earlier used versions of PACE, we started a re-examination of these evaporation residue cross section and entry state data. Results from a preliminary calculation for the reaction $136 \mathrm{MeV}{ }^{20} \mathrm{Ne}+{ }^{146} \mathrm{Nd}$ are given in Figs. IIB1.1 and IIB1.2. In this calculation we employed a simple input parameter set involving rotating liquid drop with finite range corrections yrast lines and fission barriers. 


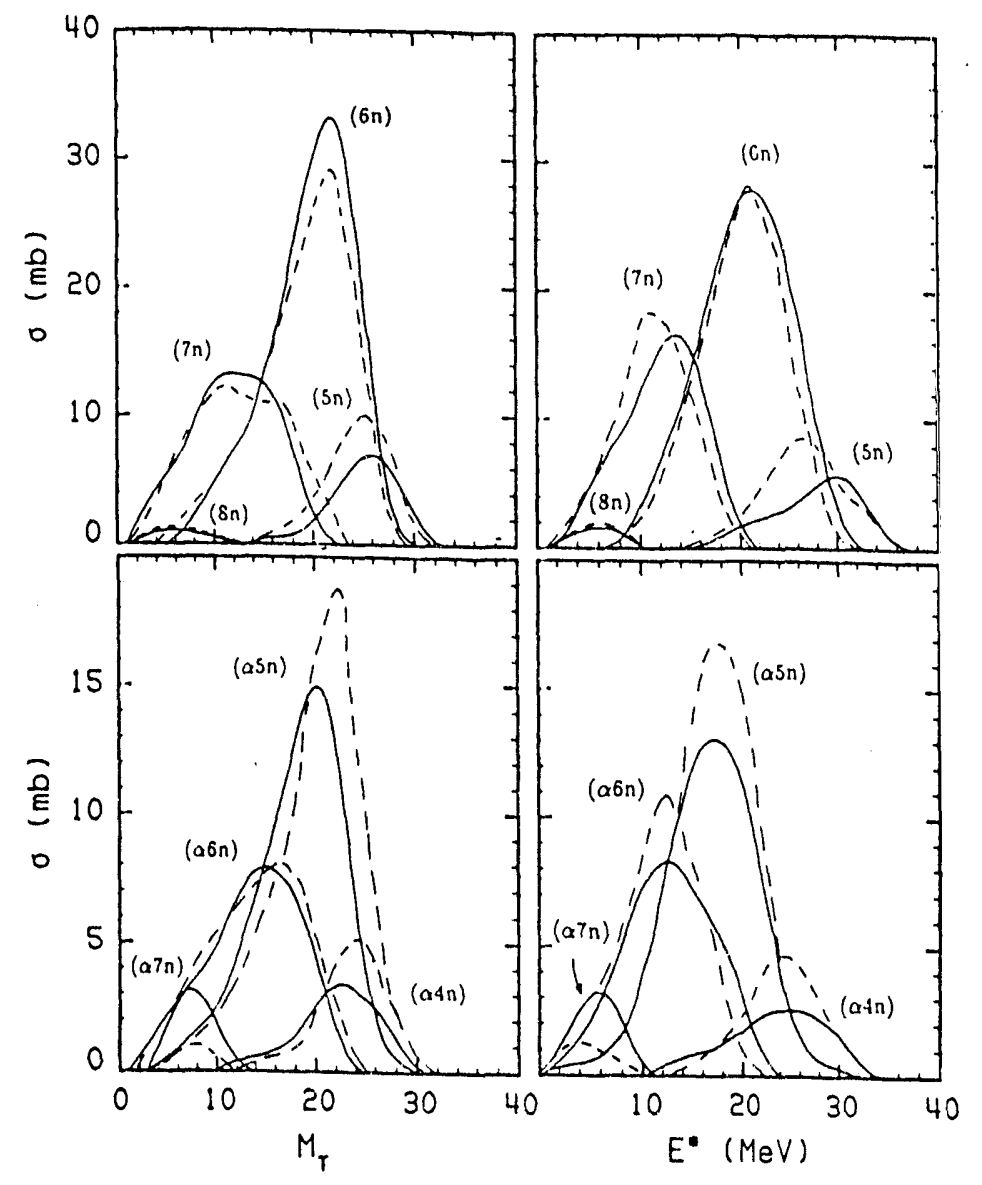

Figure IIB1.2. Comparison of the experimental (solid lines) and the calculated $\gamma$-ray multiplicity and total $\gamma$-ray energy distributions with the calculated ones (dashed lines) for the reaction 136 $\mathrm{MeV}{ }^{20} \mathrm{Ne}+{ }^{146} \mathrm{Nd}$ (See text).

The symbols in Fig. IIB1.1 show the cross sections of various evaporation residue channels in the above reaction. The result of the calculation, indicated by the histograms, is in a good agreement with the data.

A reasonably good agreement is obtained in the description of the projected entry state distributions of these channels. The solid and dashed lines of Fig. IIB1.2 show, respectively, the experimental and calculated entry state distributions as a function of the total $\gamma$-ray energy $\left(E^{*}\right)$ and gamma-ray multiplicity $\left(M_{\gamma}\right)$. The total $\gamma$-ray energy $\left(E^{*}\right)$ projections are reproduced relatively well in the peak position and absolute magnitude. The gamma-multiplicity projections agree well in shape, but they are overpredicted in absolute magnitude by two units. The dashed line $M_{\gamma}$-projections shown in Fig. 2IIB1.2 were shifted down in $M_{\gamma}$ by two units, in order to obtain agreement with the data.

Different input parameter sets are currently tested in order to explain the deviations in the prediction of the $M_{\gamma}$-projections. A possible reason for these deviations may be 
related to the fact that the experimental yrast lines of the above nuclei are steeper than the ones predicted in a liquid drop calculation.

A possible better scenario may be to use liquid-drop yrast lines for the rotational energy in the level density calculations, but realistic yrast lines consistent with the experimental ones for the $\gamma$ de-excitation part in the EVAP code. This possibility is being examined in detail and preliminary results are encouraging in improving the quantitative agreement with experiment.

Similar calculations are in progress for the $224 \mathrm{MeV}{ }^{50} \mathrm{Ti}+{ }^{130} \mathrm{Te}$ reaction, for which fission competes strongly with neutron emission.

This work was done in collaboration with the ORNL group, while all the data analysis and interpretation was done the P.I. and N. Nicolis at WU.

* Deceased.

IIB2. Nuclear Structure Effects in Proton Evaporation Spectra-D.W. Stracener, D.G. Sarantites, N.G. Nicolis, V. Abenante, Z. Majka, T.M. Semkow [WU], C. Baktash, G. Garcia-Bermudez, J.R. Beene, N.R. Johnson, M.L. Halbert, D.C. Hensley, F.K. McGowan, I.Y. Lee, M.A. Riley, A. Virtanen [ORNL], and H.C. Griffin [U. Mich].

The study of rotational motion of highly deformed nuclei is perhaps one of the best ways of studying collective motion in small quantal many-body systems. A striking example is the recent observation of superdeformed bands corresponding to a spheroidal shape with axis ratio 2:1.[1] Theoretical calculations that explain these shapes predict structures with even larger deformations ('hyperdeformed' nuclei with axis ratio $\sim 3: 1$ ) for rare earth nuclei at high spins that are near the stability limit against fission. [2] Since superdeformed nuclei are produced in very small yields in heavy-ion fusion reactions, it is important to understand the mechanisms for populating these locai minima, if ways of producing hyperdeformed nuclei are to be found. The mecharism for their population may be determined by the influence of the potential energy minima on the particle-emission probabilities via enhanced level densities and penetrabilities. These infuences will be more pronounced for charged-particle emission, because evaporation below the emission barrier is expected to be enhanced by deformation or struc :ural effects. $[3,4]$ Therefore, charged particle emission may play an important role in the population of hyperdeformed nuclei. In particular, proton emission may be more important than $\alpha$ emission, since protons do not remove much angular momentum and can probe deformation with minimal perturbation of the emitting system. These possibilities are supported by a recent report by the Chalk River group for the population of a hyperdeformed structure by a (HI,p 4 ) reaction. (Ottawa, May, 1992)

Related to this is the issue of structural influences on the shapes of the proton evaporation spectra. We have observed a strong dependence of the probability for subbarrier proton emission on the nature of the rotational bands populated in the final rucleus. 


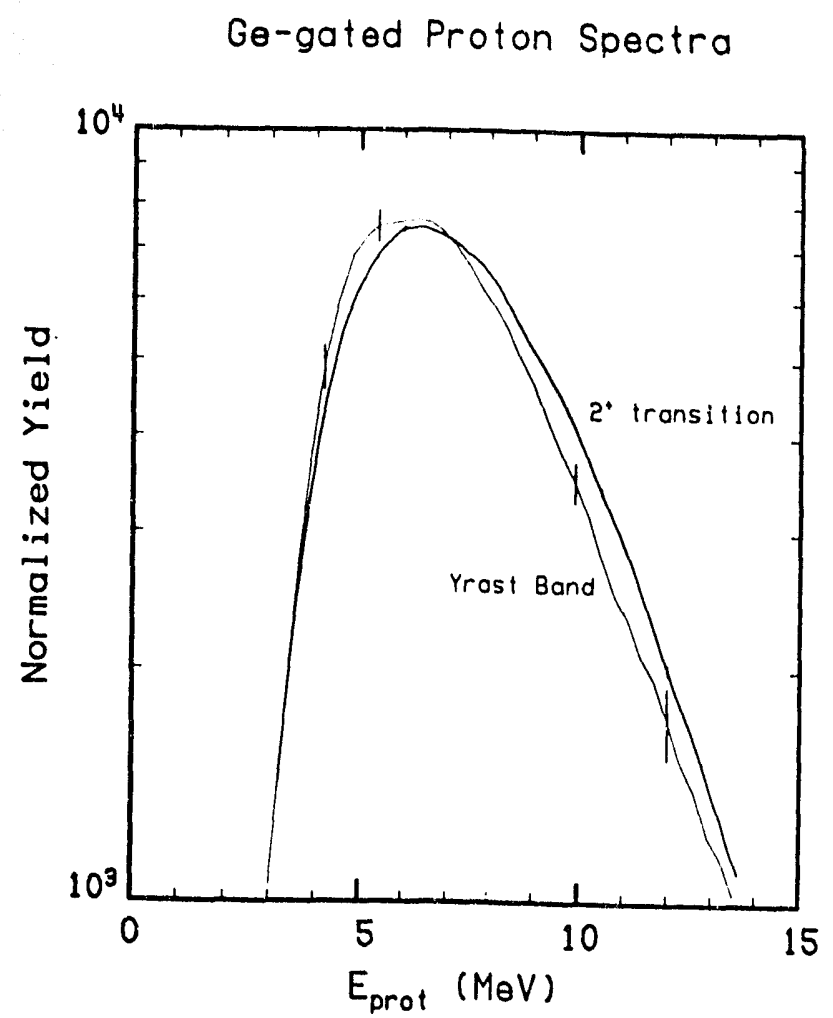

Figure IIB2.1. Proton energy spectra coincident with the $14^{+} \rightarrow 12^{+}$transition in the yrast band (thick line) and the $2^{+} \rightarrow 0^{+}$ground transition in ${ }^{82} \mathrm{Sr}$, gated by $\mathrm{k}_{\gamma} \geq 13$. The reaction was $\left({ }^{24} \mathrm{Mg}\right.$, $2 n 2 p)$.

The results to this study have been published in the Phys. Rev. Lett.[5]

Here we summarize the observations and conclusions and discuss recent measurement and calculations supporting our observations and their interpretation.

(1) As expected, the proton spectra from the $\left({ }^{34} \mathrm{~S}, 2 \mathrm{p} 2 \mathrm{n}\right)$ reaction coincident with the ground state $2^{+} \rightarrow 0^{+}$transition show a strong angular momentum dependence (sce Fig. 2a in Ref. 5) that can be understood in terms of phase space arguments from a statistical model calculation.

(2) Furthermore, the proton spectra coincident with high spin states in a given rotational band have the same shape (see Fig. 3b in Ref. 5).

(3) Large shifts are observed when proton spectra associated with different rotational bands are compared. The spectra coincident with the yrast and the ground band shift down and up in energy, respectively, when compared to the two other bands. These shifts are large, but smaller than those associated with the spin effects seen in $\gamma$-ray multiplicity gating. Fig. IIB2.1 shows a comparison of the proton spectra coincident with the $14^{+} \rightarrow 12^{+}$transition in the yrast band and the $2^{+} \rightarrow 0^{+}$transition in the ground band. The shift is of the order of $0.5 \mathrm{MeV}$. 
(4) The angular distributions of the subbarrier protons associated with the yrast band are enhanced relative to those from the $2^{+} \rightarrow 0^{+}$ground transitions which include the total channel yield. This is shown in Fig. IIB2.2.

(5) The $\gamma$-ray multiplicity distribution associated with the population of the high spin states in the yrast and the ground band are the same. This indicates that the same entry regions are involved in the population of the various rotational bands.

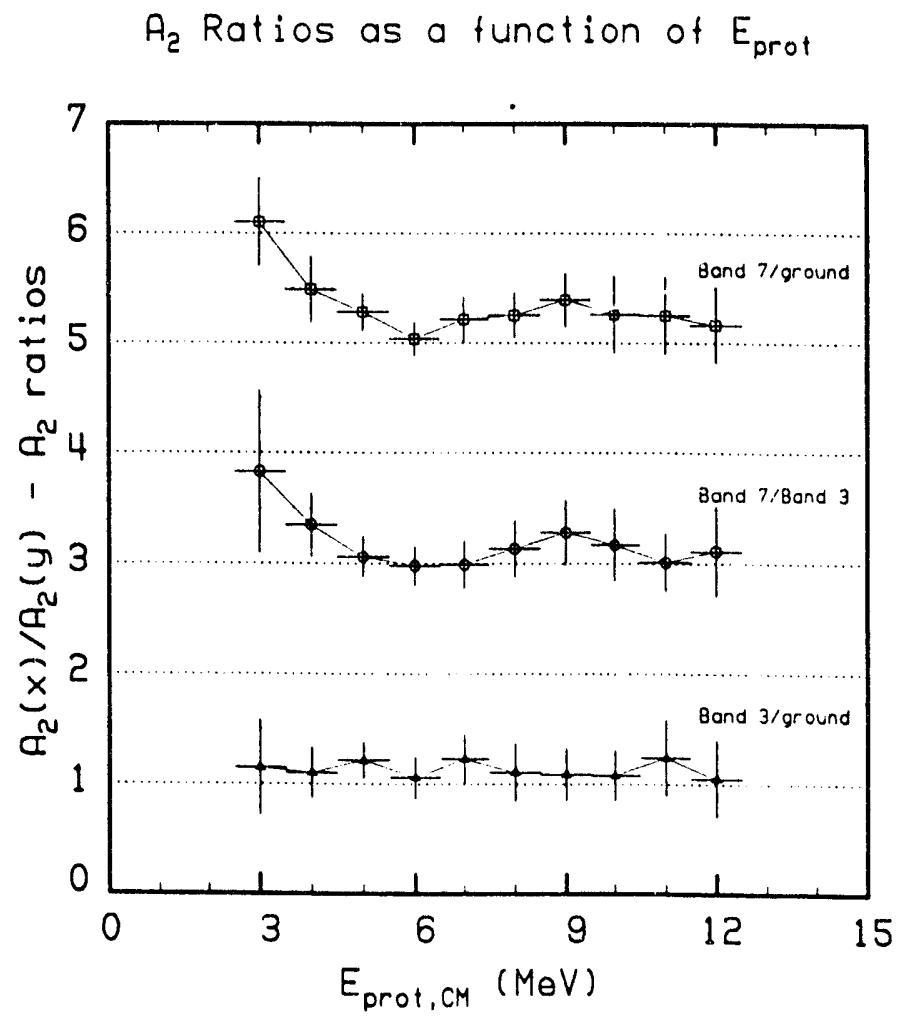

Figure IIB2.2. Ratios of angular distribution coefficients of protons coincident with the indicated rotational bands as a function of proton energy. It is seen that below the barrier $(\approx 6 \mathrm{MeV})$ the anisotropies of the protons associated with the yrast band 7 are considerably larger compared to that of the ground band.

These results are very surprising because the entry states are expected to lie at considerably higher excitation energies than the gating transitions and any correlation between the proton spectrum and the nature of the band being populated ought to be washed out by the statistical $\gamma$-ray emission.

We have carried out numerous detailed statistical model calculations in an effort to reproduce the observed shifts in the proton spectra. In all the cases either the opposite trend was found or a spin fractionation for the entry states leading to the high spin yrast and ground band was required, contrary to result (5) above.

A reasonable explanation of the observed large shifts is suggested by the steep experimental yrast line and consequently the entry line in ${ }^{82} \mathrm{Sr}$, which is only a fuw $\mathrm{MeV}$ 
above the yrast line. These observations and the data suggest that the population of the rotational bands in ${ }^{82} \mathrm{Sr}$, and in particular the yrast band, are populated by near yrast to near yrast proton emission, which significantly restricts the washing out by the statistical $\gamma$-rays of the correlation of the proton spectrum with the populated bands. This explanation is further supported by the stronger anisotropies observed (result 4 above) and by the statistical model calculations which suggest that $59 \%$ of the cascades involve a proton as the fourth emitted particle in the $\left({ }^{34} \mathrm{~S}, 2 \mathrm{n} 2 \mathrm{p}\right)$ reaction. This is shown in Fig. IIB2.3(a) where the population of the the proton emitting states leading to ${ }^{82} \mathrm{Sr}$ is shown as a density plot. The four stages of emission are visible. We further realize that according to the statistical model calculations, the subbarrier protons are by and large associated with the fourth chance emission. This is clearly seen in Fig. IIA5.1(b) where a density map is shown of the proton energy as a function of the excitation energy of the proton emitting states.
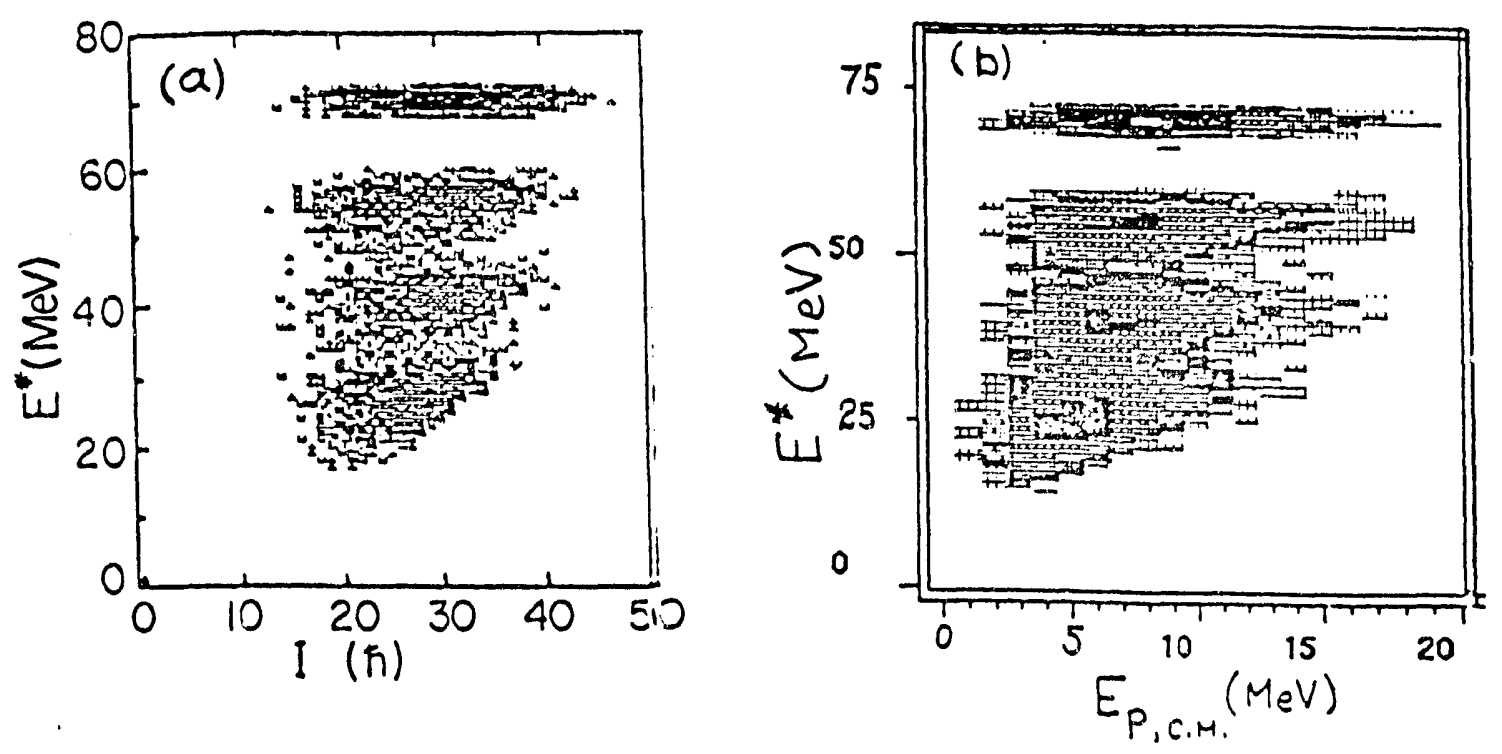

Figure IIB2.3 (a) Density plot of the proton emitting states calculated with EVAP for the reaction ${ }^{52} \mathrm{Cr}\left({ }^{34} \mathrm{~S}, 2 \mathrm{p} 2 \mathrm{n}{ }^{82} \mathrm{Sr}\right.$ at $130 \mathrm{MeV}$. A $\gamma$-ray multiplicity selection $\mathrm{M}_{\gamma}>10$ has been applied. (b) Density plot showing the energy of the emitted protons as a function of the excitation energy of the proton emitting states that lead to ${ }^{82} \mathrm{Sr}$ calculated with EVAP.

It is worth noting that in the ${ }^{82} \mathrm{Sr}$ case, proton emission from near-yrast states may be associated with a predicted mechanism based on instability toward nucleon emission of large spins, connected with the population of $h_{11 / 2}$ resonance states in nuclei in this region.[9] Those predicted yrast proton transitions were also expected to have enhanced anisotropies as observed in our work.

Since the publication of these first results, we have been anaiyzing a follow up experiment in which ${ }^{82} \mathrm{Sr}$ was produced in the reaction ${ }^{60} \mathrm{Ni}\left({ }^{26} \mathrm{Mg}, 2 \mathrm{p} 211\right)$ at $110 \mathrm{MeV}$. In this experiment several improvements were made: 
(a) The Dwarf Ball and Wall were employed, which yielded significantly more accurate angular distributions than our earlier experiment.

(b) Both $\gamma$-ray multiplicity and total $\gamma$-ray energy information were recorded with the spin spectrometer, which allow for combined $\left(\mathrm{M}_{\gamma}, \mathrm{E}_{\gamma}^{*}\right)$ gating in order to emphasize the observed effects.

(c) Better energy resolution in the Ge spectra was obtained by reducing the Doppler broadening for the high spin transitions. This was done by using a reaction with smaller recoil velocity.

The analysis of this second experiment is complete. We have made efforts to ensure that the observed effect is indeed real. With improved statistics we have examined the Ge Spectra coincident with the gates used to produce the proton spectra for impurities from other channels. The gates on the yrast band were found to contain only transitions from levels within the same band (no extra peaks were found). For the ground band a small admixture from the (HI, 2pn) channel was found and this accounts for part of the observed upward shift in the proton spectra coincident with this band.

We note that the (HI, $2 \mathrm{p} 3 \mathrm{n}$ ) channel is not present in these gates, nor is it populated significantly in these reactions. Any other contamination will produce a shift in the opposite direction (upward shift) to the protons associated with the yrast band. We have also found that the proton energ. shift occurs only for gates on transitions originating from levels with spins higher than $10^{+}$.

We have examined the dependence of the shift on the combined total energy $(\mathrm{H})$ and multiplicity gating $\left(k_{\gamma}\right)$. Whereas the multiplicity dependence is very strong, only minor shifts were observed for constant $k_{\gamma}$ for different $\mathrm{H}_{\gamma}$ values. The shapes of the $\gamma$-ray continuum spectra associated with the gates that show the proton shift are significantly different. Those associated with the yrast band show a softer statistical $\gamma$-ray component.

The experiments were done at HHIRF with assistance from the ORNL group. All the data analysis and interpretation was done by Dan Stracener and the P.I at WU. This is part of Stracener's PhD. thesis.

1. P.J. Nolan and P.J. Twin, Ann. Rev. Nucl. Part. Sci. 38, 533 (1988).

2. J. Dudek in 'The l'amety of Nuclear Shapes', ed. J.D. Garrett et al. (World Scientific, 1987), p. 195.

3. M. Blann and T.T. Komoto, Phys. Scr. 24,93 (1981).

4. J.M. Alexander, D. Guerreau and L.C. Vaz, Z. Phys. A305, 313 (1982).

5. D.G. Sarantites et al. Phys. Rev. Lett. 18, 2129 (1990).

6. Z. Majka et al., Phys. Rev. Lett. 58, 322 (1987).

7. J.B. Natowitz, Nucl. Phys. A482, 171c (1988).

8. F.A. Dilmanian et al., Phys. Rev. Lett. 49, 1909 (1982).

9. N.G. Nicolis et al., Phys. Rev. C41, $2118(1990)$.

10 T. Dossing, S. Frauendorf, and II. Schulz, Vucl. Phys. A287, 137 (1977).

11. G.A. Leander, Comp. Phys. Comm. 47, 311 (1987). 
IIB3. Nuclear Structure Dependent Entry State Population by Total Spectroscopy - A.S. Kirov, D.G. Sarantites, J. Barreto, R.J. Charity, L. Gallamore, J. Muñoz, N.G. Nicolis, L.G. Sobotka, D.W. Stracener [WU], D.C. Hensley, C. Baktash, J.R. Beene, I.Y. Lee, F.K. McGowan, M.L. Halbert, and D. Winchell [ORNL]

The purpose of this experiment is to extend previous studies of structure effects observed in proton evaporation spectra [1] to a new reaction ${ }^{58} \mathrm{Ni}\left({ }^{20} \mathrm{Si}, 2 \mathrm{p} \alpha\right){ }^{81} \mathrm{Sr}$. Structure dependent effects were reported in the previous section, where shifts as large as $0.5 \mathrm{MeV}$ in the proton spectra were seen when the ground or the yrast bands are populated at high spin via the ${ }^{52} \mathrm{Cr}\left({ }^{34} \mathrm{~S}, 2 \mathrm{n} 2 \mathrm{p}\right){ }^{82} \mathrm{Sr}$ reaction. Such effects were claimed [2], [3] to be smaller than $160 \mathrm{keV}$ in the following different reactions ${ }^{58} \mathrm{Ni}\left({ }^{29} \mathrm{Si}, 2 \mathrm{pn}\right){ }^{84} \mathrm{Zr}$ and ${ }^{58} \mathrm{Ni}\left({ }^{29} \mathrm{Si}, 2 \mathrm{p} \alpha\right){ }^{81} \mathrm{Sr}$.

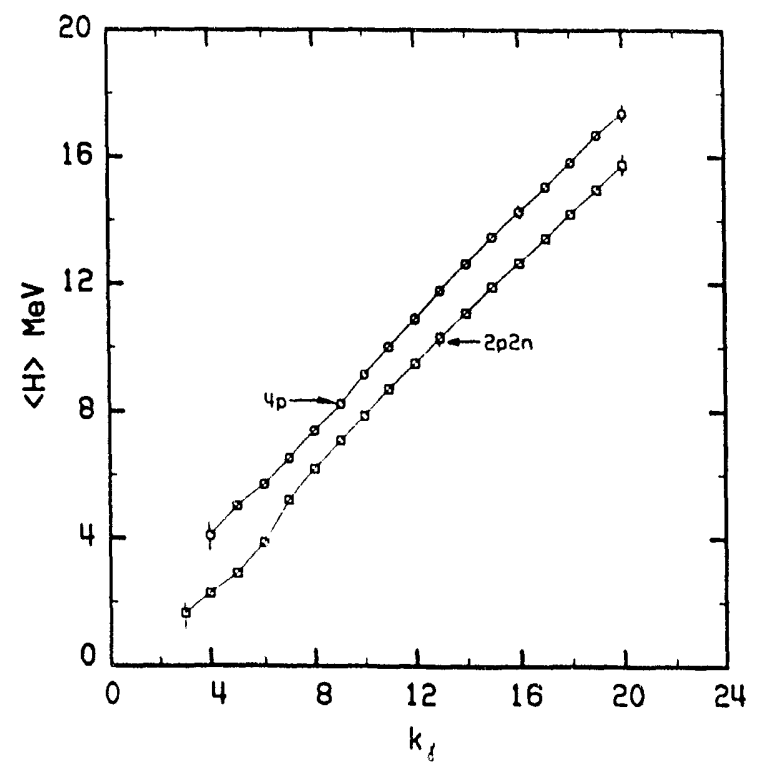

Figure IIB3.1. Entry lines of total average $\gamma$-energy $\left\langle\mathrm{H}_{\gamma}\right\rangle$ as a function of $\mathrm{k}_{\gamma}$ from the $4 \mathrm{p}$ and $2 \mathrm{p} 2 \mathrm{n}$ experiments corresponding to the $2^{+}-0^{+}$transition in ${ }^{82} \mathrm{Sr}$.

In order to verify our previous results and extend our studies to more complete measurements, we studied the population of the same residual nucleus ${ }^{82} \mathrm{Sr}$ via a different reaction : ${ }^{58} \mathrm{Ni}\left({ }^{28} \mathrm{Si}, 4 \mathrm{p}\right)^{82} \mathrm{Sr}$ by employing a new concept of "total spectroscopy" as follows. By identifying all 4 protons and measuring their energies we can determine directly the excitation energy of the entry states from where the $\gamma$-cascades initiate.

The experiment was performed at the HHIRF. The detection systems were the "Dwarf Ball and Wall" (consisting of 103 CsI(T) ) detectors, the "Spin Spectrometer" (with 63 out of 72 scintillation detectors in place), and plus 18 Compton suppressed Ge(Li) detectors. This setup allowed us to perform total spectroscopy of ${ }^{82} \mathrm{Sr}$ by measuring the discrete transitions identifying the various rotational bands, the $\gamma$-ray multiplicity (a measure of the input spin of the entry states) and the excitation energy of the entry states by directly 


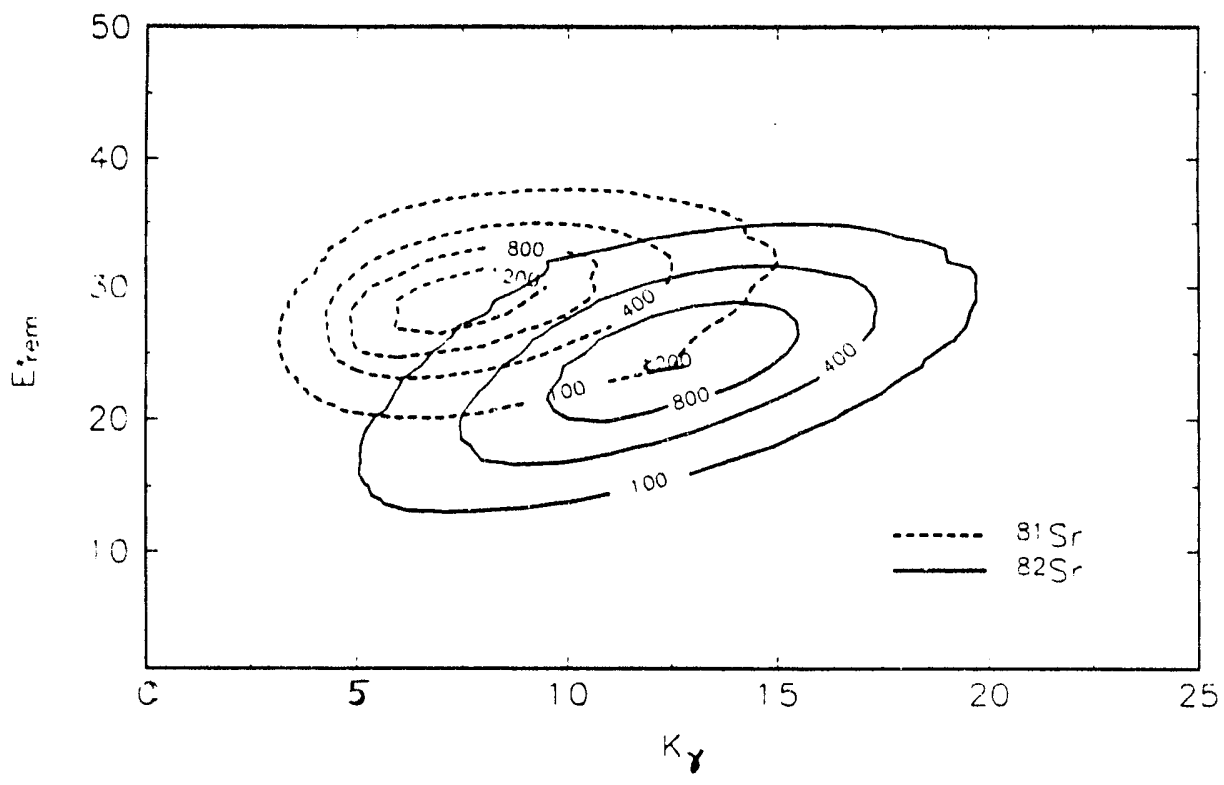

Figure IIB3.2. Contour plots of the remaining energy $\mathrm{E}_{r e m}^{*}$ after the emission of $4 \mathrm{p}$ (solid lines) and of $4 \mathrm{pn}$ vs. $\mathrm{k}_{\gamma}$ coincident with transitions in ${ }^{82} \mathrm{Sr}$ and ${ }^{81} \mathrm{Sr}$, respectively.

measuring all the particles. The latter is considerably more accurate than the total $\gamma$ ray energy, which is incomplete. We chose the projectile energy (130 MeV), to give an excitation energy of $69.2 \mathrm{MeV}$ in the compound nucleus that matches that in our previous measurements.

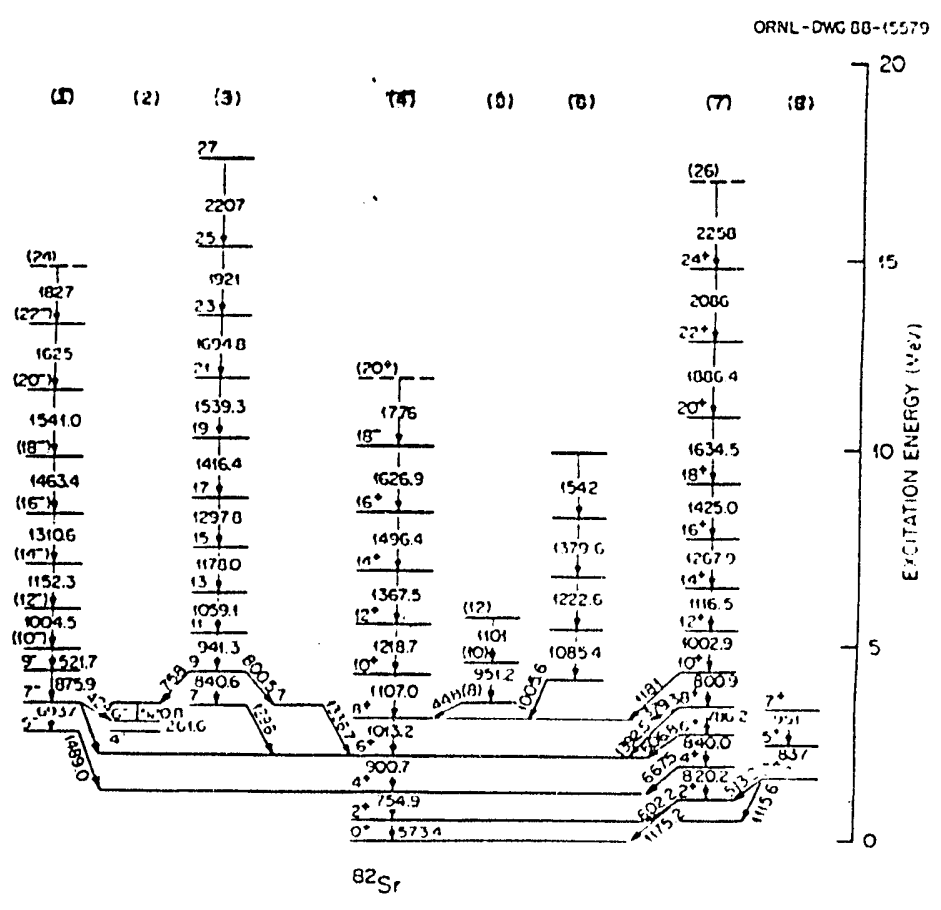

Figure IIB3.3. Level scheme of ${ }^{82} \mathrm{Sr}$. 
Figure IIB3.4. (a) Contour plots of $\mathrm{E}_{\text {rem }}^{*}$ vs. $\mathrm{k}_{\gamma}$ coincident with $4 \mathrm{p}$ and the ground state transition $2^{+} \rightarrow 0^{+}$ (dash dot curves) and the transitions from tive $7^{+}$and $17^{+}$of band 3 in ${ }^{82} \mathrm{Sr}$. (b) Samie as in (a) but for the $2^{+} \rightarrow 0^{+}$ground transition (solid lines) and the $14^{+} \rightarrow$ $12^{+}$transition in band 4 . (c) Same as above but coincident with the $14^{+} \rightarrow 12^{+}$transitions in bands 4 (solid lines) and 7 (dash-dot curves), respectively. The contours correspond to outward decreasing levels of $0.8,0.6,0.4$, and 0.2 of the maxinum in each case.
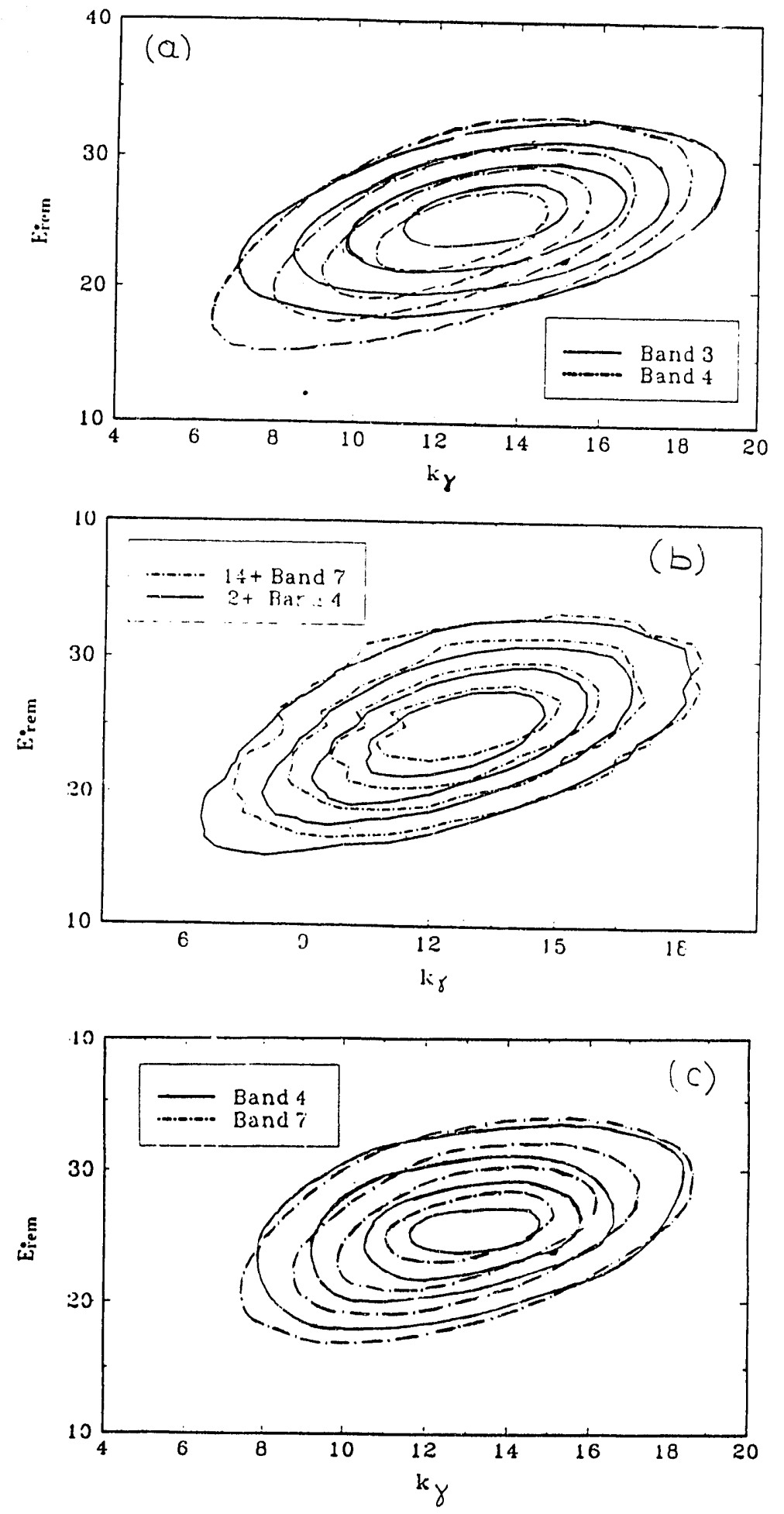

In these experiments the Dwarf system and the Spin Spectrometer were always in the trigger. In the first $1 / 3$ of the run 2 or more Ge were $r$ quired in the trigger while for the 


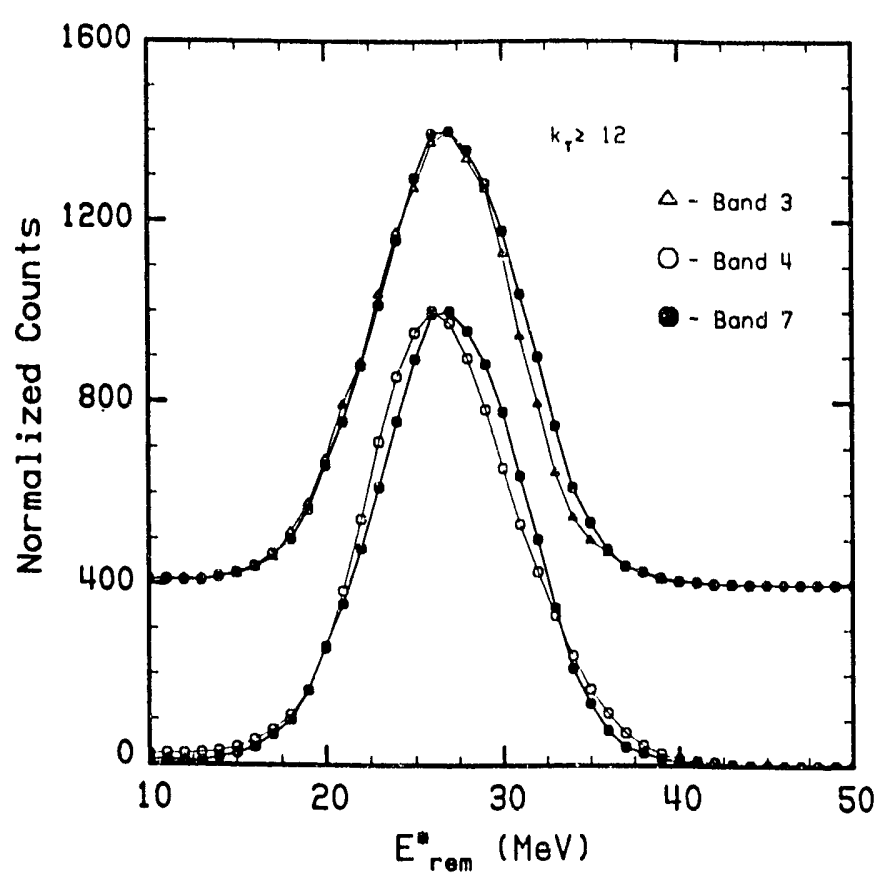

Figure IIB3.5. Excitation energy distributions corresponding to $k_{\gamma} \geq 12$ coincident with $4 \mathrm{p}$ and the $14^{+} \rightarrow 12^{+}$transitions in band 4 (open circles, lower part) and band 7 (closed circles, lower and upward shifted parts), and the $17 \rightarrow 15$ transition (open triangles) in band 3 . The excitation energy shifts are quite apparent.

remainder 1 or more were required. The 2-Ge trigger data (2.7 million $4 \mathrm{p}$ events) allowed us to construct a $\gamma-\gamma$ coincidence matrix for checking the purity of the Ge gates, while the 1-Ge triggered data ( 11 million $4 p$ events) increased the total statistics considerably. In this experiment the $4 \mathrm{p}$ exit channel events were $\sim 4 \%$ of the total.

Plots of the total $\gamma$-energy, $\mathrm{H}_{\gamma}$, vs. the $\gamma$-fold, $\mathrm{k}_{\gamma}$, were built and compared to those from the ${ }^{52} \mathrm{Cr}\left({ }^{34} \mathrm{~S}, 2 \mathrm{n} 2 \mathrm{p}\right)^{82} \mathrm{Sr}$ reaction. To avoid possible interference from contaminating channels, we gated on the $2^{+}-0^{+}$transit on in ${ }^{82} \mathrm{Sr}$. For easier comparison instead of the entry populations we show in Fig. $\mathrm{IiP}^{2} \perp$ the entry lines $\left\langle\mathrm{H}_{\gamma}\right\rangle$ as a function of $\mathrm{k}_{\gamma}$ for these two reactions. Although these two reactions have similar $\ell_{f u s}$ values of 50 and $49 \hbar$, it is seen that the entry line from the present $4 \mathrm{p}$ experiment is higher by $\sim 1.2 \mathrm{MeV}$ for the same $\mathrm{k}_{\gamma}$, or lower by $1 \mathrm{k}_{\gamma}$ unit for the same $\left\langle\mathrm{H}_{\gamma}\right\rangle$. Furthermore, for the transition to the ground state in the $2 \mathrm{p} 2 \mathrm{n}$ and the $4 \mathrm{p}$ reactions the total $\left\langle\mathrm{H}_{\gamma}\right\rangle$ is 10.68 and 11.26 $\mathrm{MeV}$, respectively, while $\left\langle\mathrm{k}_{\gamma}\right\rangle$ is 12.5 and 11.4 , respectively.

This difference suggests that the structure dependent shifts in the proton spectra should be less pronounced in the 4 p reaction $[2]$ compared to those from the $2 \mathrm{p} 2 \mathrm{n}$ reaction[1]. Thus we might expect the band structure should exhibit a weaker connection to the entry region for the $4 \mathrm{p}$ case compared to the $2 \mathrm{p} 2 \mathrm{n}$. The observed lower spin population can be explained by assuming that the 4 protons carry away more angular momentum than 2 protons and 2 


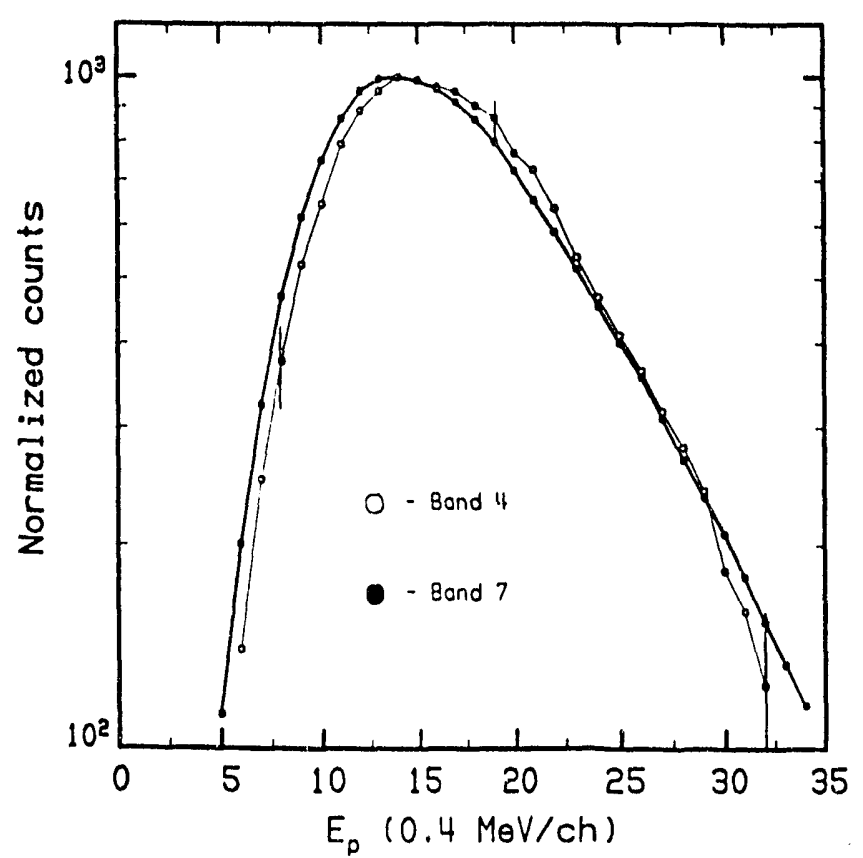

Figure IIB3.6. Proton spectra coincident with $4 \mathrm{p}$ and the $14^{+} \rightarrow 12^{+}$transitions in bands 4 (open circles) and 7 (closed circles) corresponding to $k_{\gamma} \geq 12$.

neutrons. This is reasonable since the kinetic energy of the emitted protons is higher than that of the evaporated neutrons due to the Coulomb barrier. The same argument would most probably apply for other reactions in which structure effects were not seen, since these reactions have predominantly emission of charged particles in the exit channels: $3 \mathrm{p}$, $2 \mathrm{p} \alpha$, and 2pn. $[2,3]$

The concept of total spectroscopy provides a powerful method for study of these reactions. The determination of the entry region for the $\gamma$-cascade is possible now by directly calculating the remaining energy in the ${ }^{82} \mathrm{Sr}$ residual nucleus from the measured kinetic energy of the 4 protons. If we assumed that charged particle emission precedes $\gamma$ emission then this remaining energy is the excitation energy in the residue. In Fig. IIB3.2 is shown a contour plot of the remaining energy for the $4 \mathrm{p}$ channel as a function of $k_{\gamma}$. The data presented by the solid contours are additionally gated by the $2^{+} \rightarrow 0^{+}$transition in ${ }^{82} \mathrm{Sr}$. The dashed contours correspond to gates on four transitions in three bands of ${ }^{81} \mathrm{Sr}$, the $4 \mathrm{pn}$ product. The elevated position of the ${ }^{81} \mathrm{Sr}$ contours reflects the fact that the energy removed by the neutron is not measured. The two regions are clearly different. This displacement helps identify any contamination in the weak high spin transitions in ${ }^{82} \mathrm{Sr}$. This method is considerably more sensitive for identifying small contaminations compared to gating on the $\gamma-\gamma$ matrix and examining the coincident Ge spectra. As a further check for contaminant transitions in the $\gamma$-gates, Ge spectra were built for each relevant Ge gate on transitions on ${ }^{82} \mathrm{Sr}$. The gates contaminated with ${ }^{81} \mathrm{Sr} \gamma$ rays showed up more sensitively 

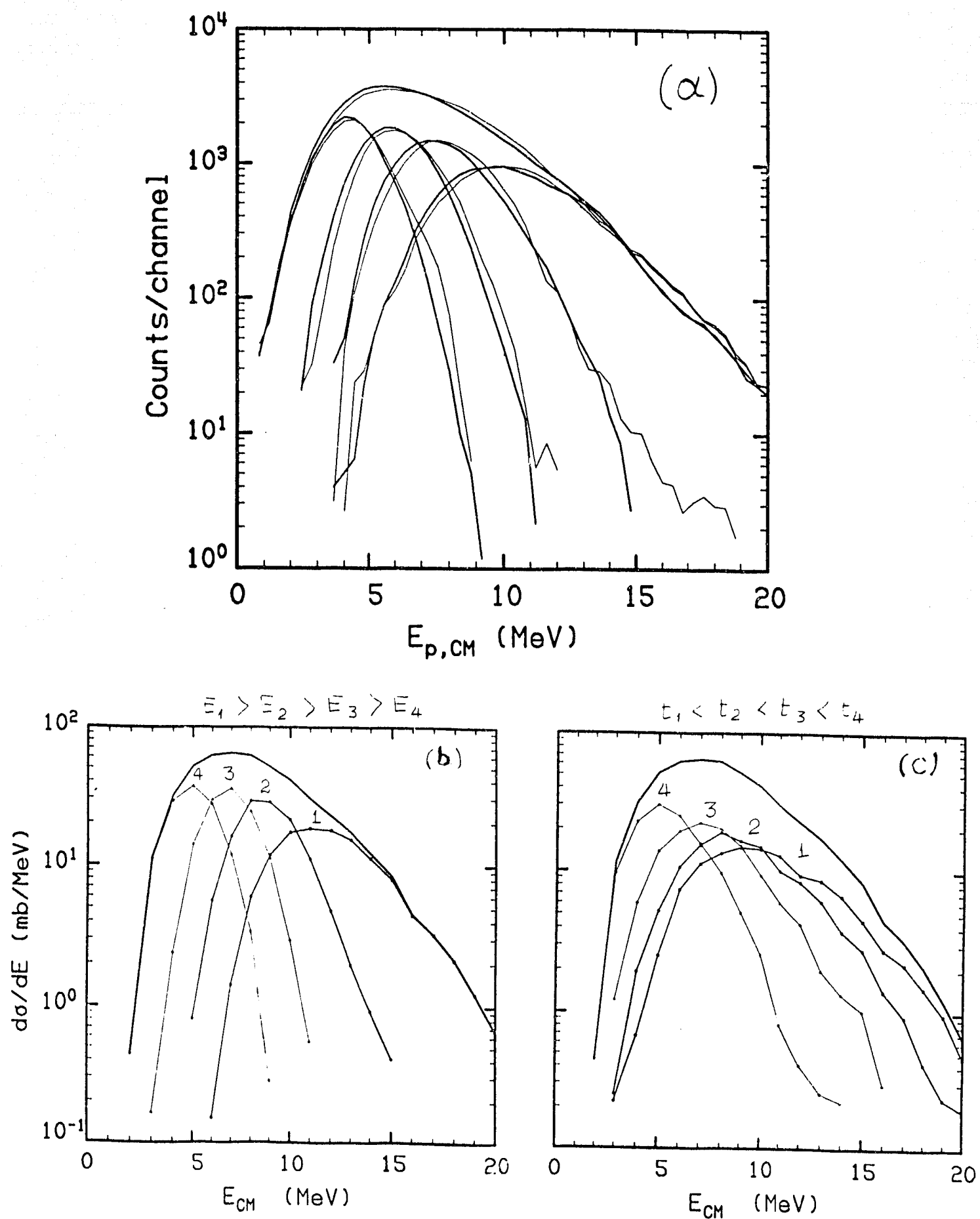

Figure IIB3.7. (a) Energy-ordered experimental proton energy spectra coincident with $4 \mathrm{p}, \mathrm{k}_{\gamma} \geq 12$ and the $14^{+} \rightarrow 12^{+}$transition in bands 4 (thin lines) and 7 (thick lines) in ${ }^{82} \mathrm{Sr}$, respectively. The total proton spectra are also shown. The energy shifts are present in both the energy ordered spectra and the total spectra. (b) and (c) Energy and time ordered proton spectra for the $4 \mathrm{p}$ exit channel from a Monte Carlo simulation of the reaction with the evaporation code EVAP. It is seen that the $4^{\text {th }}$ chance protons are strongly correlated with the lowest energy protons. 
in the $\mathrm{E}_{\gamma e m}^{*}$ vs. $\mathrm{k}_{\gamma}$ map. The higher sensitivity is due to the fact that the contamination appears in a background free region.

Contour maps of $E_{r e m}^{*}$ vs. $k_{\gamma}$ were constructed for clean transitions in the 4 strongly populated bands of ${ }^{82} \mathrm{Sr}$ (see Fig. IIB3.3 for the decay scheme). All gates were checked for contamination with ${ }^{81} \mathrm{Sr}$ both by the $\gamma-\gamma$ matrix method and the $\left(\mathrm{E}_{r e m}^{*}, \mathrm{k}_{\gamma}\right)$ map method. Contamination from other channels, i.e. $5 \mathrm{p}\left({ }^{81} \mathrm{Rb}\right)$ or $3 \mathrm{p} \alpha\left({ }^{79} \mathrm{Rb}\right)$ was also excluded, since none of the known transitions in these products could be seen. To correct each map corresponding to a $/$ gate for the underlying Compton background, the map from a nearby background was subtracted. Smoothing by adjusting the middle point to the least squares fit over a number of neighbouring points ( 7 or 9 ) was used in most cases.

The $\left(\mathrm{E}_{r e m}^{*}, \mathrm{k}_{\gamma}\right)$ maps corresponding to the $17 \rightarrow 15$ transition in band 3 and the $2^{+} \rightarrow 0^{+}$in band 4 are compared in Fig. IIB3.4a. It is seen that the map for band 3 is somewhat rotated clockwise and shifted toward higher $\mathrm{k}_{\gamma}$ values compared to the ground transition. A similar effect but different in magnitude is observed for the map associated with the $14^{+} \rightarrow 12^{+}$transition in band 4 compared to the ground state transition. In contrast, the map associated with the $14^{+} \rightarrow 12^{+}$of the yrast band 7 does not rotate but only shifts somewhat toward higher $\mathrm{E}_{r e m}^{*}$ and $\mathrm{k}_{\gamma}$ values compared to the ground transition as seen in Fig IIB3.4b. Furthermore, the entry populations associated with the $14^{+} \rightarrow 12^{+}$ transition in bands 4 and 7 are different. The map of band 7 shifts mainly toward higher $\mathrm{E}_{\text {rem }}^{*}$ values as seen in Fig. IIB3.4c. Examples of these excitation energy shifts is shown in Fig. IIB3.5. Here the excitation energy distributions (or $\mathrm{E}_{r e m}^{*}$ ) are plotted for $\mathrm{k}_{\gamma} \geq 12$ as projections from Figs. IIB3.4a,c. It is again seen that the excitation energies that lead to band 7 are higher than those that lead to bands 4 or 3 at states of similar high spin and excitation.

These effects were first observed as differences in the proton spectra coincident with high spin transitions in the various bands. To indicate the magnitude of these differences we show in Fig. IIB3.6 the total proton spectra that lead to the $14^{+}$states of band 4 (open circles) and the yrast band 7 (closed circles) when $a k_{\gamma} \geq 12$ selection was applied. The proton spectrum for band 7 shifts down by $0.45 \pm 0.08 \mathrm{MeV}$.

The above structure dependent differences in the entry regions that populate various bands can be understood in terms of the persistence of significant similarity in the structure above the yrast line that the $\gamma$-decay pathways do not complete wash out the each band character. We are planning at present model calculations using the code GAMBLE that will include some structure information in order to understand these effects in some detail.

Another informative aspect of the total spectroscopy is look at the energy ordered proton spectra from the $4 \mathrm{p}$ reaction that lead to the various rotational bands. These are constructed by making four spectra in which for each event we histogram the ordered energies to the corresponding spectra. This is shown in Fig. IIB3.7a for the spectra leading to the $14^{+}$of bands 7 (thick lines) and band 4 (thin lines). The downward displacements of the spectra from band 7 are quite apparent. The energy ordering of the spectra bears 
an interesting correlation to the time ordering of the 4 emitted protons. To illustrate this correlation we show in Figs. IIB3.7b,c the energy and time ordered proton spectra from a Monte Carlo simulation of the spectra for this reaction using the code EVAP. From this comparison we learn that the spectrum of the lowest energy proton is at least in the subbarrier region that of the last emitted proton. Unfortunately, the highest two energy ordered spectra have substantial mixing between first and second chance emission.

This experiment was done at HHIRF with assistance from Bob Charity and L. Gallamore (Sobotka'a group). All the data analysis and interpretation was done by Assen Firov and the P.I at WU. This is part of Firov's PhD. thesis.

[1] N.G. Nicolis, et al. Nucl. Phys. A 520, 153 (1990).

[2] K.Pohl et al., Bull. Am. Phys.Soc. 37, 936 (1992).

[3] D.J.Blumenthal et al., Phys. Rev. Let.t. 66, 3121 (1991).

IIB4. Entrance Channel Effects in Fusion Near the Barrier - J. Barreto, D.G. Sarantites, R.J. Charity, N.G. Nicolis, L. Gallamore, D.W. Stracener, L.G. Sobotka, [WU], J.R. Beene, C. Baktash, M.L. Halbert, D.C. Hensley [ORNL], and M. Thoennessen [MSU].

The experimental observation[1-7] that the decay modes of excited compound nuclei appear to depend on their mode of formation is one of the most unexpected findings of heavy ion fusion reactions near the Coulomb barrier. Entrance channel effects were observed[1-3] by comparing ratios of $x n$ and/or $\alpha x n$ evaporation cross sections as a function of the compound nucleus spin distribution, formed in different HI reactions. Specifically, large differences were observed in the exit-channel cross sections at high spin. In additional experiments, large differences were also found in fission cross sections[4], and neutron multiplicities $[2,5,6]$, as a function of entrance channel mass asymmetry. Entrance channel effects have also been reported[[] for the $\gamma$-decay in the region of the GDR and in the population of superdeformed bands[8,9], for compound nuclei formed in symmetric and asymmetric entrance channels.

Trapping in a superdeformed minimum for the mass symmetric entrance channel was proposed $[1,7,10]$ as an explanation for these observed effects. If this is correct, one would expect an enhanced emission of low energy $\alpha$-particles, due to the lowering of the Coulomb barrier for that configuration. However, this is contrary to the observation[3] of a larger $\alpha$-particle emission cross section, in the $\alpha 2$ n evaporation channol for ${ }^{164} \mathrm{Y} b$ at $\mathrm{E}^{*}=49$ $\mathrm{MeV}$, for the ${ }^{16} \mathrm{O}+{ }^{148} \mathrm{Sm}$ case, when compared to that from the ${ }^{64} \mathrm{Ni}+{ }^{100} \mathrm{Mo}$ reaction. It can also be argued that an incomplete fusion contribution might be responsible for the larger $\alpha$-particle cross section observed in the ${ }^{16} \mathrm{O}$ induced reaction.

Clearly, a satisfactory understanding of these effects is still lacking, and the full understanding of the origin of these entrance channel effects needs more theoretical and experimental work. In order to shed some light on this subject, we have performed a 
detailed study in the decay of ${ }^{164} \mathrm{Yb}$ at $\mathrm{E}^{*}=52 \mathrm{MeV}$ formed by the ${ }^{64} \mathrm{Ni}+{ }^{100} \mathrm{Mo}$ and ${ }^{16} \mathrm{O}$ $+{ }^{148} \mathrm{Sm}$ reactions. We measured spectra and angular distributions of charged particles in coincidence with discrete $\gamma$-ray transitions that identify the residual nuclei. In addition, $\gamma$-ray multiplicity distributions were measured for both the $\alpha \times n$ and the xn exit channels also also identified by the respective discrete $\gamma$-ray transitions.

The discrete $\gamma$-rays were detected with 18 Compton suppressed Ge detectors, the charged particles were detected with the Dwarf Ball, and the $\gamma$-ray multiplicity was obtained with the Spin Spectrometer.

The shapes of the charged particle spectra, and their angular distributions, in conjunction with the $\gamma$-ray fold distributions should be a sensitive probe for the previously observed entrance channel effects in the decay of the compound nucleus formed in heavy ion fusion reactions near the Coulomb barrier.

Some results of this work have been presented previously[11] (see Appendix G), and it is part of a more general study currently been undertaken by our group in unraveling the origin of any entrance channel effects in the decay of the compound nucleus. The work undertaken here extends a previous study[12] where we studied the decay of ${ }^{164} \mathrm{Yb}$ at a lower excitation energy.

There are two aspects that we shall address here. The first deals with the large displacement in spin for the the population of the $\alpha \mathrm{n}$ channel for the symmetric and asymmetric entrance channels [1]. The issue is whether this is due to nonequilibrium effects in the fusion process or other influences such the breakdown of Bohr's independence hypothesis. The second aspect is in the purported[1] difference in the decay mode following the $2 \mathrm{n} / 3 \mathrm{n}$ exit channel cross section ratio as a function of spin. The origin of this difference has been ascribed to the proximity of the nearby second minimum in the potential energy surface that leads to the superdeformation. A more unpleasant scenario would be that there might be an alternate much more natural explanation associated with the mechanism of the measurement (not necessarily an error).

In order to address the first aspect, we shall examine spectra and angular distributions of $\alpha$ particles associated with the $\alpha 2 \mathrm{n}$ and $\alpha 3 \mathrm{n}$ channels from the ${ }^{16.4} \mathrm{Yb}$ decay at $52 \mathrm{MeV}$, when formed by the ${ }^{64} \mathrm{Ni}+{ }^{100} \mathrm{Mo}$ and the ${ }^{16} \mathrm{O}+{ }^{128} \mathrm{Sm}$ reactions.

Representative $\alpha$ particle spectra in coincidence with $\mathrm{E}_{\gamma}=192.1 \mathrm{keV}$ transition in ${ }^{158} \mathrm{Er}+\alpha 2 \mathrm{n}$ exit channel are shown in Fig. IIB4.1 for both the $\mathrm{Ni}$ and $\mathrm{O}$ reactions, at three selected CM angles. These spectra were obtained by summing the individual spectra for all detectors in the same ring (same $\theta_{l a b}$ ). For both reactions, $\alpha$ particle center of mass energies were measured from 13-30 $\mathrm{MeV}$ (the exit channel Coulomb barrier is around 19 $\mathrm{MeV})$.

Figure IIB4.2 shows charged-particle CM angular distributions, obtained by integrating the corresponding measured energy spectra, for each of the two reactions. In the ${ }^{64} \mathrm{Ni}+$ ${ }^{100}$ Mo system (Fig. IIB4.2a), the $\alpha 2 \mathrm{n}$ and $\alpha 3 \mathrm{n}$ are the only charged particle decay channels with measurable particle yields in coincidence with discrete $\gamma$-ray transitions. For ${ }^{16} \mathrm{O}+$ 


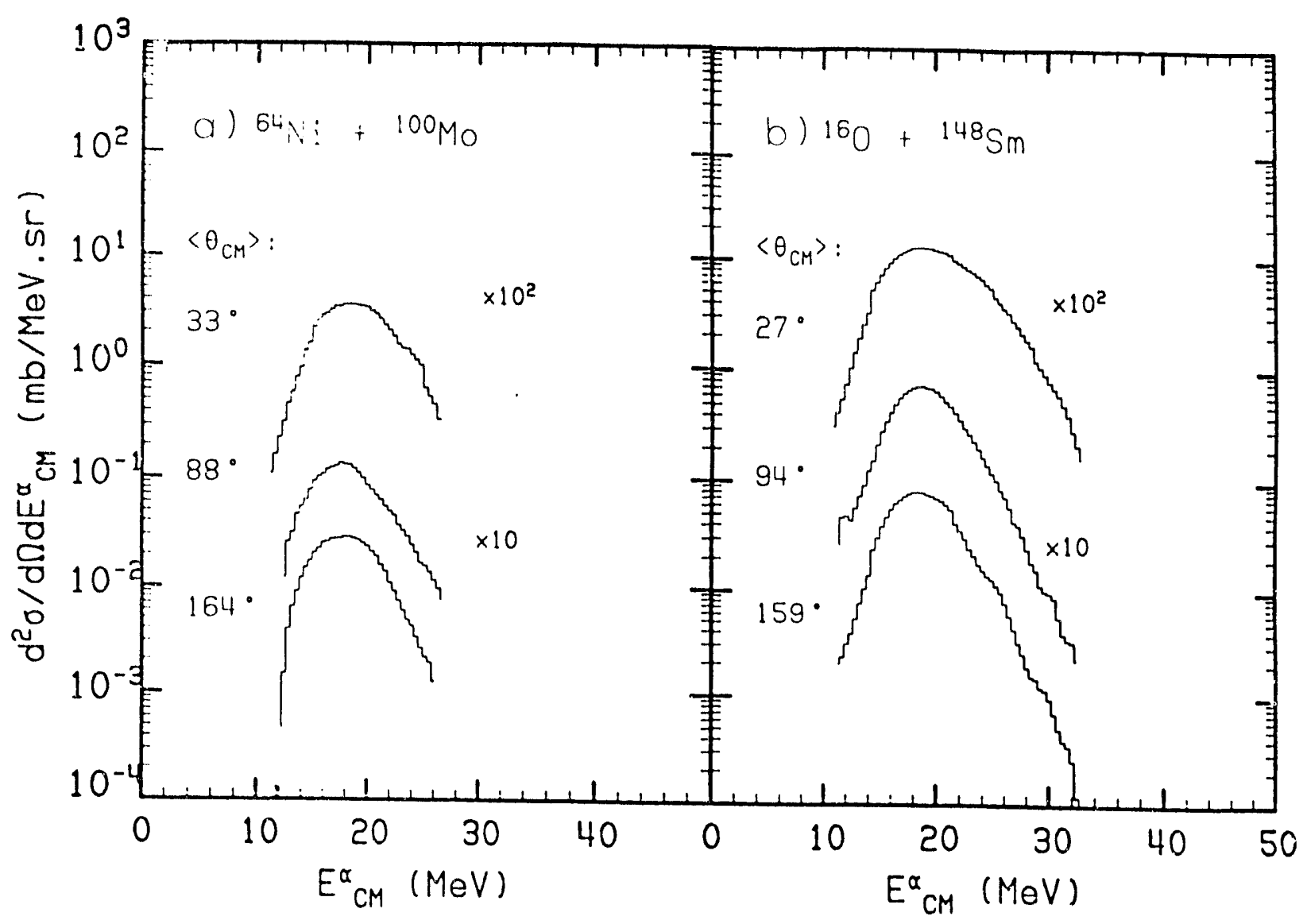

Fig. IIB4.1. Alpha particle double differential cross sections $\left(\mathrm{d}^{2} \sigma / \mathrm{d} \Omega \mathrm{d} \mathrm{E}_{\alpha}\right)_{C M}$ for the $\alpha 2 \mathrm{n}$ exit channel as a function of $\mathrm{E}_{\mathrm{C}, \mathrm{W}}^{\alpha}$, at the indicated $\mathrm{CM}$ angles, for (a) ${ }^{64} \mathrm{Ni}+{ }^{100} \mathrm{Mo}$ and (b) ${ }^{16} \mathrm{O}+$ ${ }^{148} \mathrm{Sm}$ reactions.

${ }^{148} \mathrm{Sm}$ (Fig. IIB4.2b), besides these two channels, the ${ }^{161} \mathrm{Tm}+\mathrm{p} 2 \mathrm{n}$ channel could be measured.

In the ${ }^{64} \mathrm{Ni}+{ }^{100} \mathrm{Mo}$ system, the angular distributions show a pattern that is symmetric around $\theta_{C M}=90^{\circ}$ for both channels (Fig. IIB4.2a), which is characteristic of emission from a fully equilibrated compound nucleus. In the ${ }^{16} \mathrm{O}+{ }^{148} \mathrm{Sm}$ system, however, the angular distributions for the $\alpha 2 \mathrm{n}$ and $\alpha 3 \mathrm{n}$ channels exhibit rather different patterns which are strongly forward peaked. Surprisingly, the p2n channel shows a pattern that is consistent with emission from an equilibrated compound nucleus.

This indicates that non-statistical mechanisms are contributing only to the $\alpha 2 \mathrm{n}$ and $\alpha 3 \mathrm{n}$ exit channels. This forward peaked component is stronger for the $\alpha 2 \mathrm{n}$ channel. Furthermore, there is a strong dependence of the angular distributions on $\mathrm{E}_{\alpha}$. Angular distributions of $\alpha$ particles from the $\alpha 2 \mathrm{n}$ decay channel are shown in Fig. IIB4.3, for the indicated center-of-mass energy bins from the ${ }^{16} \mathrm{O}$ reaction. The forward contribution in the angular distribution is clearly associated with high energy $\alpha$-particles. Furthermore, as indicated in Fig. IIB4.3b, these high energy $\alpha$-particles are associated with the lowest $\gamma$-ray folds. This non-equilibrium component is responsible for the shift of the associated 

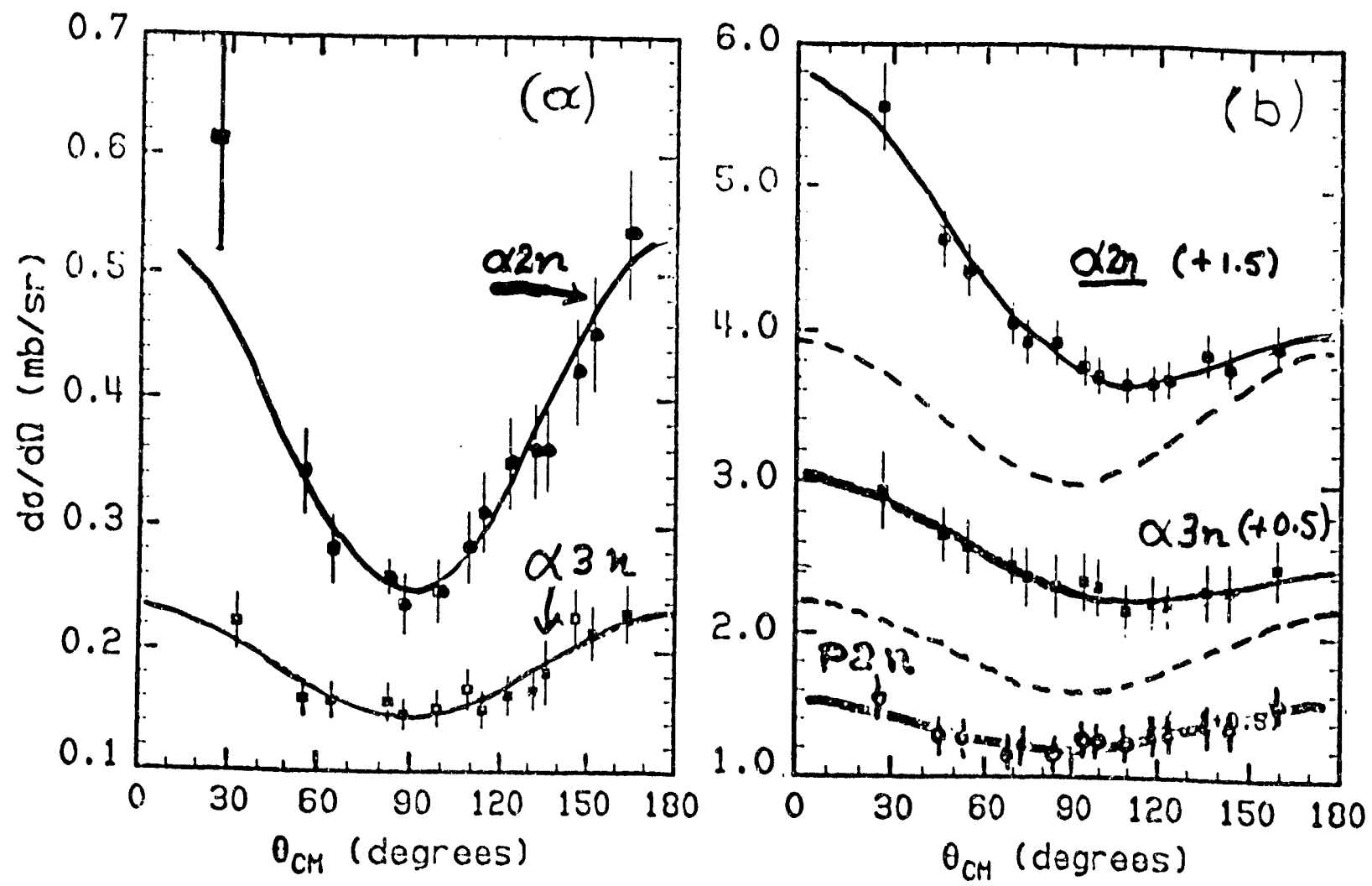

Fig. IIB4.2. Center of mass angular distributions for the reactions (a): ${ }^{64} \mathrm{Ni}+{ }^{100} \mathrm{Mo}$ and (b): ${ }^{16} \mathrm{O}$ $+{ }^{148} \mathrm{Sm}$. The open squares are for the $\alpha 2 \mathrm{n}$, the closed squares for the $\alpha 3 \mathrm{n}$, and the open circles for the p2n decay channels, respectively. The thick solid curves in (a) represent fits of Eq. (1) to the $\alpha 2 \mathrm{n}$ and $\alpha 3 \mathrm{n}$ data. In (b) the thick curves give an estimate of the equilibrium component for the $\alpha x n$ channels. For the p2n channel, the thick curve is a fit of Eq. (1) to the data.

$\gamma$-ray multiplicities to lower values. These results are consistent with the breakup of the ${ }^{16} \mathrm{O}$ projectile into a ${ }^{12} \mathrm{C}+{ }^{\alpha}$, followed by fusion of the ${ }^{12} \mathrm{C}$ with the target nucleus.

Needless to say, that as far as the axn channels are concerned the two reactions do not correspond to the formation of compound nuclei at the same excitations. Thus, the reaction dynamies are different and there is no need to talk about entrance channel effects.

In contrast, the $\alpha$-particle angular distributions from the ${ }^{64} \mathrm{Ni}+{ }^{100} \mathrm{Mo}$ reaction can be fitted with a Legendre polynomial equation of the type:

$$
W\left(\theta_{C M}\right)=A_{0}\left[1+A_{2} P_{2}\left(\cos \theta_{C M}\right)\right] .
$$

The decomposition of the forward peaked angular distributions from the ${ }^{16} \mathrm{O}+{ }^{148} \mathrm{Sm}$ reaction is not a simple matter. An attempt for such a decomposition is shown by the dashed lines in Fig. IIB4.2b, where anisotropies consistent with those from the Ni reaction attenuated somewhat to reflect the spin difference were kept constant while the forward component was represented by a Legendre polynomial expansion with $P_{1}$ and $P_{3}$ terms. 

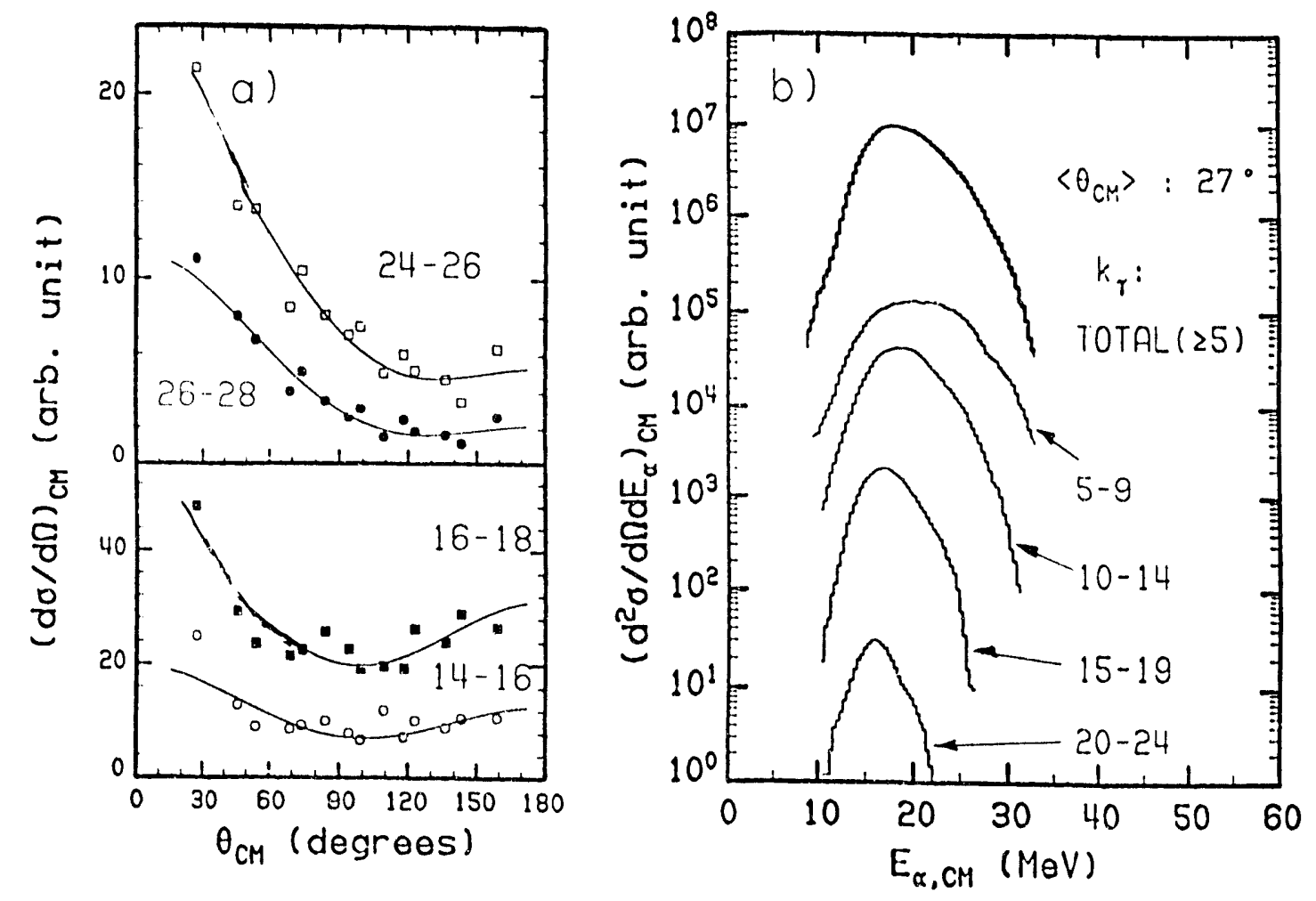

Fig. IIB4.3. (a) Center of mass angular distributions of $\alpha$-particles for the $\alpha 2 \mathrm{n}$ channel from the ${ }^{16} \mathrm{O}+{ }^{148} \mathrm{Sm}$ reaction for the indicated $\alpha$-energy bins. See Fig. IIB4.2b for the meaning of the solid curves. (b) $\alpha$-particle spectra measured in the most forward ring for the same reaction, and its decomposition using the indicated $\gamma$-ray coincidence gates.

The formation of longer lived superdeformed configurations at high spin, in the symmetric system, has been suggested $[1,3,5,7]$ as a possible explanation for the effects observed in the previous experiments. If this shape configuration exists in the ${ }^{64} \mathrm{Ni}+{ }^{100} \mathrm{Mo}$ channel, an enhancement of the subbarrier $\alpha$-particle emission should be observed in this system, with the angular distribution showing a large anisotropy at sulbbarrier energies[17,18]. Fig. IIB 4.4a shows $\alpha$-particle angular distributions for the $\alpha 2 \mathrm{n}$ channel formed in the ${ }^{6 \cdot \mathrm{Ni}}+{ }^{100} \mathrm{Mo}$ reaction, for 3 different $a$-particle center of mass energy bins. These angular distributions were fitted to Eq. (1) and the $A_{2}$ coefficients are plotted in Fig. IIB4.4b as a function of $\mathrm{E}_{\alpha}$. The variation of the experimental anisotropy with the $\alpha$-particle energy, in the subbarrier region, shows a pattern that is characteristic of emission from a spherical system. No evidence for a large shape deformation is found for the ${ }^{64} \mathrm{Ni}+{ }^{100} \mathrm{Mo}$ system using this probe.

The second aspect of the present study deals with the $\gamma$-ray fold distributions for the xn and the $\alpha \times n$ exit channels. These distributions were measured with the Spin Spectrometer. The left panels in Fig. IIB4.5 show the experimental cross-sections for various xn decay channels as a function of the $\gamma$-ray fold distribution $k_{e f f}[16]$, for the ${ }^{16} \mathrm{O}(\mathrm{top})$ and ${ }^{64} \mathrm{Ni}$ (bottom) induced reactions. Besides the fact that the cross sections for the $3 \mathrm{n}$ and $4 \mathrm{n}$ exit 

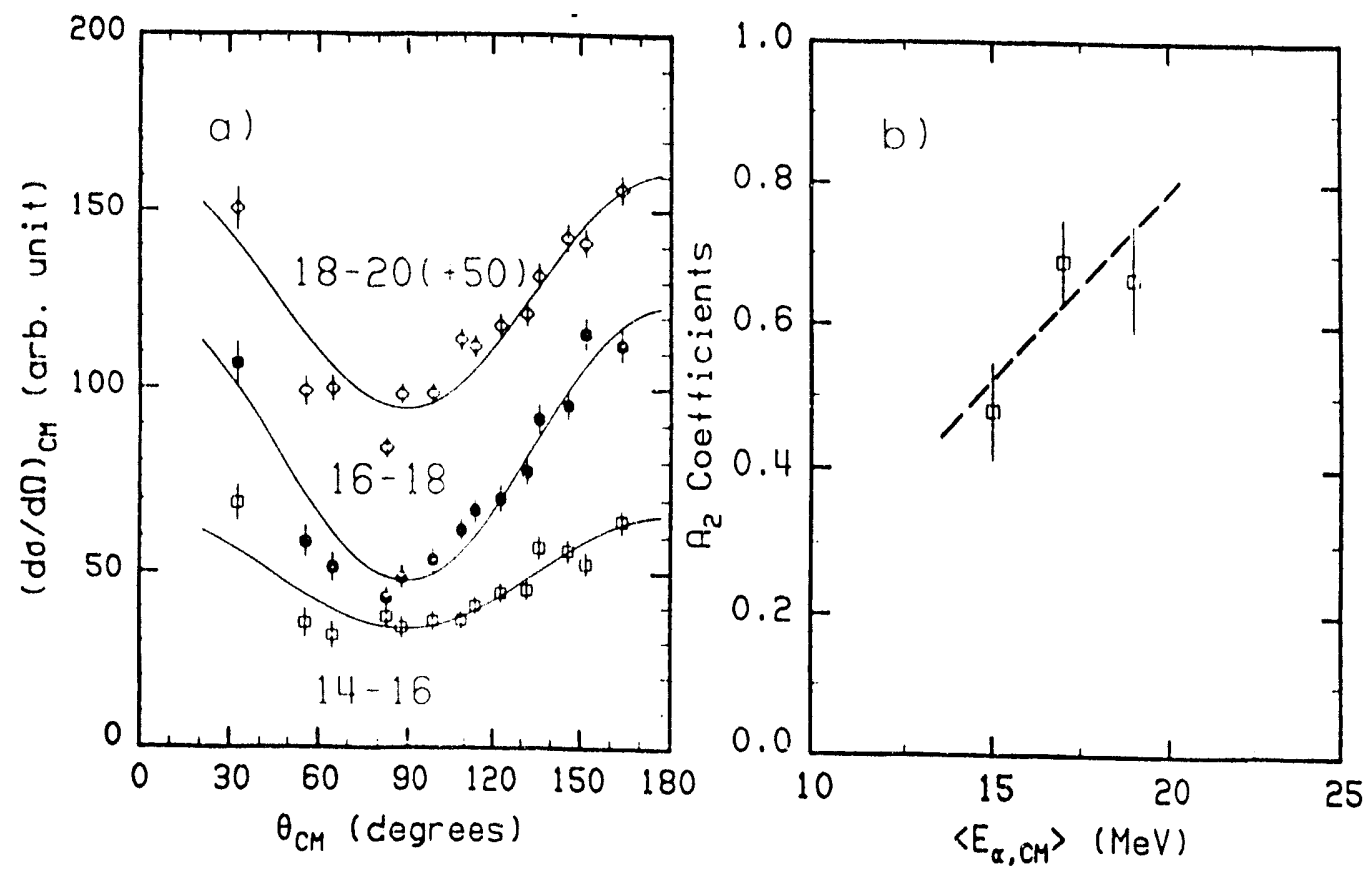

Fig. IIB4.4. (a) Center of mass angular distributions for the $\alpha 2 \mathrm{n}$ exit channel in the case of the ${ }^{64} \mathrm{Ni}+{ }^{100} \mathrm{Mo}$ reaction, gated by various $\alpha$-particle subbarrier energy bins. See Fig. IIB4.2a for the meaning of the solid lines. (b) Extracted $\mathrm{A}_{2}$ angular distribution coefficients as a function of the corresponding average $\alpha$-particle energy.

channels from ${ }^{16} \mathrm{O}+{ }^{148} \mathrm{Sm}$ are larger compared to those from ${ }^{64} \mathrm{Ni}+{ }^{100} \mathrm{Mo}$, an effect very similar to that reported previously[1.3] is observed at the higher $k_{\gamma}$ values reached in the ${ }^{16} \mathrm{O}+{ }^{148} \mathrm{Sm}$ : an apparent shift in the population of the $3 \mathrm{n}$ and $2 \mathrm{n}$ channels toward lower $k_{\gamma}$ values is observed which is largest for the $2 \mathrm{n}$ channel.

Quantitatively, from Fig. IIBt.j (left panels) we see that although the $k_{\gamma}$ distributions for the $4 \mathrm{n}$ channel have nearly the same shape and position for both reactions, the $3 \mathrm{n}$ channel is shifted down by 2 to 3 units from the ${ }^{16} \mathrm{O}+{ }^{148} \mathrm{Sm}$ reaction, with the yield extending to values below $10 \mathrm{in} \mathrm{k}_{y}$. This effect is more pronounced in the $2 \mathrm{n}$ decay channel, where a shift as large as 5 units is observed. This is highlighted in Fig. IIB4.6, where the cross-section fractions $\sigma_{i}(\mathrm{k}) / \Sigma \sigma_{i}(\mathrm{k})$ for the $2 \mathrm{n}, 3 \mathrm{n}$ and $4 \mathrm{n}$ channels are plotted as a function of $k_{e f f}$. Substantial differences are seen at all $k_{\gamma}$ values above $\sim 7$, but the most dramatic appears to be the difference for the $2 \mathrm{n}$ channel. These differences could be interpreted as evidence for entrance channel effects. In principle, however, the independence of the decay on entrance channel should be checked by such comparisons in terms of the compound nucleus spin. The fact that $k_{\gamma}$ is monotonically related to spin needs to be considered in detail as it will done below.

In the case of light charged particles, the $k_{e f f}$ distributions are shown also in Fig. IIB4.5 (right panels) for $\alpha 2 \mathrm{n}, \alpha 3 \mathrm{n}$ and $\mathrm{p} 2 \mathrm{n}$ decay channels for all charged particle energies 

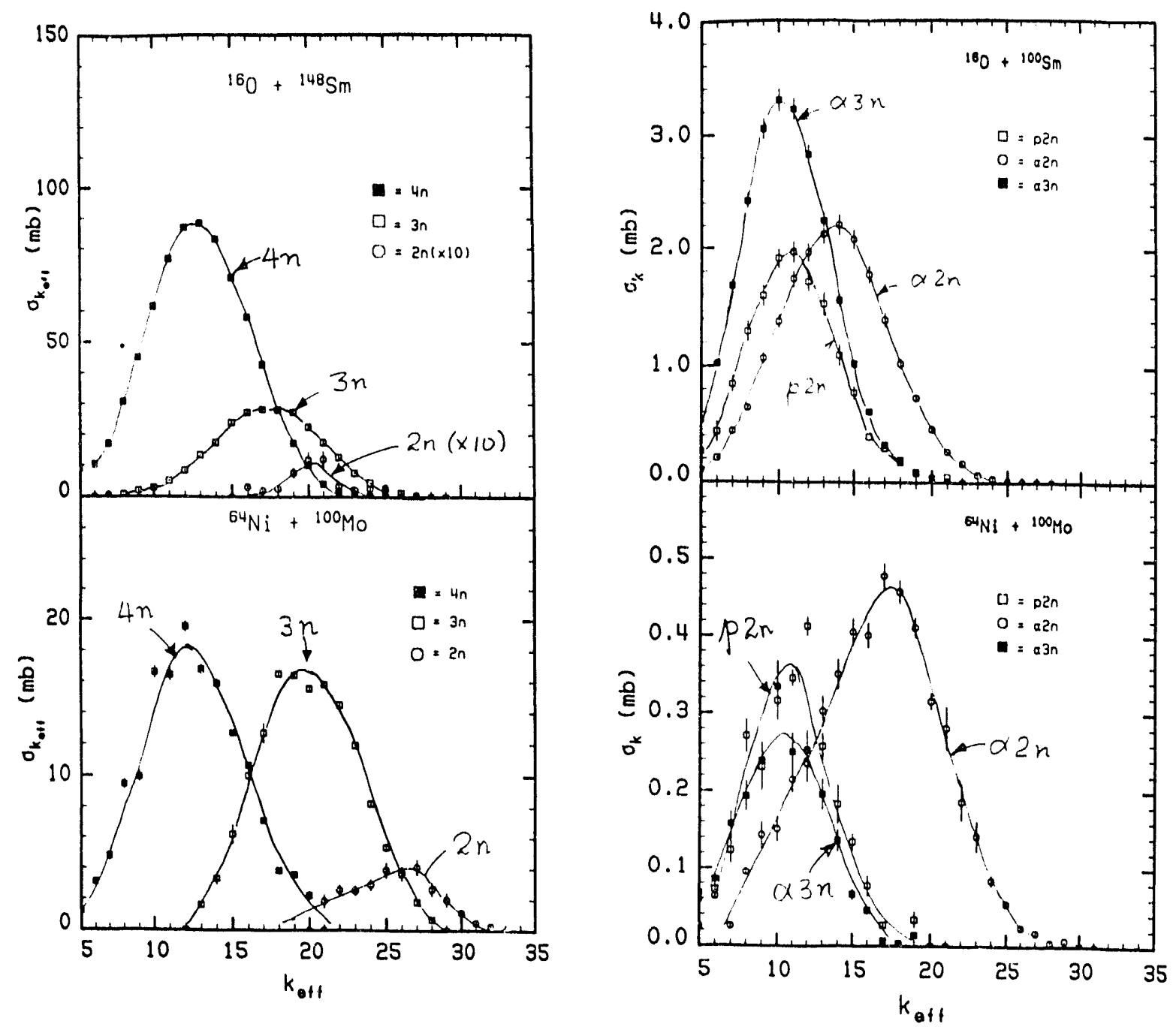

Fig. IIB4.5. Channel cross-sections as a function of $k_{e f f}$ for the $2 n, 3 n$ and $4 n$ exit channels (left panels) from the decay of ${ }^{164} \mathrm{Yb}$ at $\mathrm{E}^{a}$ * of $52 \mathrm{MeV}$ from ${ }^{16} \mathrm{O}+{ }^{148} \mathrm{Sm}$ (top) and ${ }^{64} \mathrm{Ni}+{ }^{100} \mathrm{Mo}$ (bottom ). Similarly for the $\alpha 2 \mathrm{n}, \alpha 3 \mathrm{n}$ and p2n channels (right panels) for the ${ }^{16} \mathrm{O}$ (top) and ${ }^{64} \mathrm{Ni}$ (bottom) induced reactions.

and for both entrance reactions. First, we note that the p2n channel is from equilibrated decays and the $k_{\gamma}$ distributions are similar in shape and position. The $k_{\gamma}$ distribution for the $\alpha 2 \mathrm{n}$ channel shows33 the largest downward shift, which can be explained quantitatively as due to the ${ }^{16} \mathrm{O}$ breakup as discussed earlier. Similarly, the $\alpha 3 \mathrm{n}$ channel shows a smaller downward shift for the ${ }^{16} \mathrm{O}$ induced reaction, which is consistent with the observed smaller breakup probability for the projectile. We are at present attempting a quantitative decomposition of the $\mathrm{k}_{\gamma}$ distributions in terms of the two reaction mechanisms.

Now we turn to the issue of the $\mathrm{k}_{\gamma}$ distributions for the xn channels. In order to make meaningful comparisons for entrance channel effects one must deduce the spin distributions 

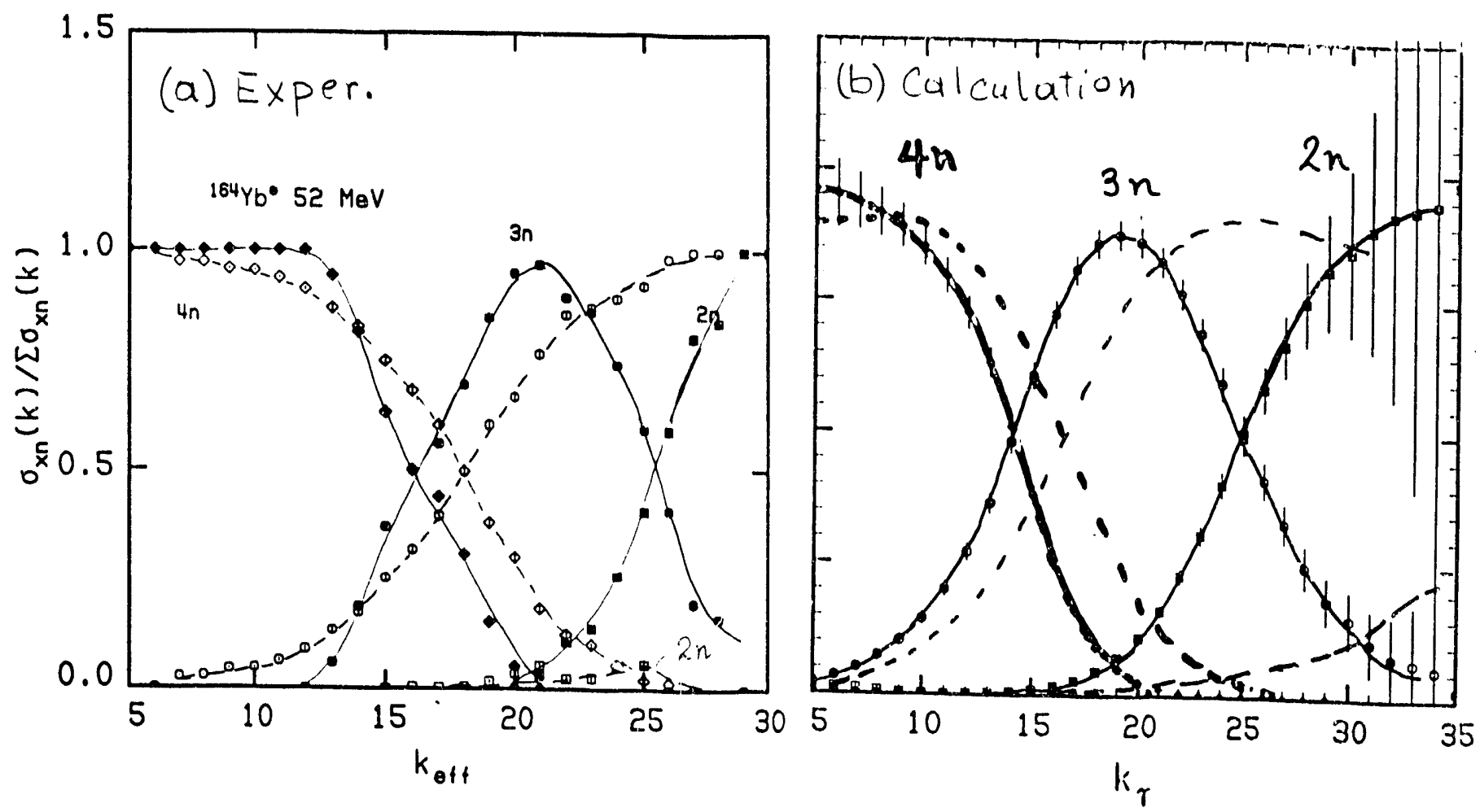

Fig. IIB4.6. (a) Experimental channel cross-section fractions $\sigma_{i}(\mathrm{k}) / \Sigma \sigma_{i}(\mathrm{k})$ for the $2 \mathrm{n}, 3 \mathrm{n}$ and $4 \mathrm{n}$ channels, as a function of $\mathrm{k}_{\text {eff }}$ (see text). Closed and open symbols refer to the ${ }^{64} \mathrm{Ni}$ and ${ }^{16} \mathrm{O}$ induced reactions, respectively. (b) The same as in (a) but calculated by a Monte Carlo simulation with the code EVAP. Qualitative agreement is observed (see text).

from the $k_{\gamma}$ ones. This was done by previous workers [1] using techniques that we developed 17 years ago. Such reconstructions were meant for average values and are not suited for distributions. Here we will take a different approach. We shall use realistic reaction models to produce initial $\ell$ distributions that lead to fusion, and then via statistical model calculations with the code EVAP we shall derive the spin distributions in each residue, and then the $\gamma$-ray multiplicity, $\mathrm{M}_{\gamma}$, distributions for each residue will be obtained. Finally, from the latter the $k_{\gamma}$ distributions will be calculated using the measured $\mathrm{M}_{\gamma} \rightarrow \mathrm{k}_{\gamma}$ responses for the spin spectrometer. The resulting $k_{\gamma}$ distributions are then compared with experiment. This procedure avoids problems with consecutive unfoldings which are not always unique. The initial $\ell$ distributions may be altered until agreement with experiment is reached.

We have done the above in a series of calculations. For the $\mathrm{Ni}+$ Mo reaction we use an $\ell$ distribution from a channel coupling calculation of Landowne and Dasso[19] and 
a diffuseness of 2 for the $\mathrm{O}+\mathrm{Sm}$ case. From these calculations the $\mathrm{M}_{\gamma}$ distributions were obtained and then multiplied by the Spin Spectrometer responses from $M_{\gamma}$ to $k_{\gamma}$. Then the fractions for each $x n$ exit channel were calculated as a function of $k_{\gamma}$. The results are shown in Fig. IIB4.6b. It is seen that the differenes purported to be entrance channels effects from Fig. IIB4.6a are surprisingly well reproduced without any specific assumption on different decay mechanisms. We can understand this to be due to the spreading of the initial $\ell$ distributions due to the three consecutive mappings that are applied $\left(\ell \rightarrow I_{\text {res }} \rightarrow M_{\gamma} \rightarrow k_{\gamma}\right)$. To illustrate this consider the population of the $2 \mathrm{n}$ channel in $\ell$ which is a very narrow distribution due to the cutoff at the top of the total $\ell$ distribution. This is spread out considerably (mainly by the first 2 steps) so that values well above the mean are mainly due to spreading. For the $\mathrm{Ni}+$ Mo reaction, however, there is real cross for these $\ell$ values because the latter reaction populates higher $\ell$ values. Thus differences produced due to spreading should not be interpreted as entrance channel effects. In this trivial way we can explain all previous claimed entrance channel effects in the xn exit channels.

We conclude by observing that the bulk of previously clained entrance channel effects are due either to differences in the reaction dynamics between the symmetric and asymmetric entrance channels or due to the limitations of the experiments which give the $k_{\gamma}$ distributions instead of those in $\ell$. When the proper mechanisms for producing the compound nuclei are introduced and the decay is properly followed by a statistical model calculation the experimental features can be well reproduced without need to resort to strong structural influences on the deexcitation of the compound nucleus by particle emission.

This experiment was done at HHIRF with assistance from Bob C'harity and L. Gallamore (Sobotka'a group). The data analysis and interpretation were done exclusively by Jorge Barreto, and N. Nicolis of our group.

[1] A. Ruckelshausen et al., Phys. Rev. Lett. 56, 2356 (1986).

[2] D.J. Love et al., Phys. Rev. Lett. 57, 5:51 (1986).

[3] J. R. Beene, M. Thoennessen, and M.L. Halbert, ORNL Progr. Report, 60 (1990-1991), and private communication.

[1] F.L.H. Wolfs et al., Phys. Rev. (39, 865 (1989).

[5] W. Kühn et al. Phys. Rev. Lett. 51, 1858 (198:3).

[6] W. Kühn et al., Phys. Rev. Lett. 62, $1103(1989)$.

[7] M. Thoennessen et al., Proc. of the Workshop on Vuclear Structure and Heavy-ion Reaction Dynamics, Inst. Phys. Conf. Ser. No. 109, Chapter 5, 135 (1990).

[8] G. Smith et al., Phys. Rev. Lett. 68,158(1992).

[9] S. Flibotte et al., Phys. Rev. (45, $889(1992)$.

[10] H. Feldmeier, Rep. Prog. Phys. 50, 915 (1987).

[11] J.L. Barreto et al., Proc. of the Eight Winter Workshop on Nuclear Dynamics, Jackson Hole - WY, $18-25,(1992)$.

[12] M.L. Halbert et al., Phys. Rev. $(40,2558(1989)$.

[13] D.W. Stracener et al., Nucl. Inst. Meth. A 294, 485 (1990).

[14] M. Jääskeläinen et al., Nucl. Inst. Meth. 204, 385 (1983).

[15] K. Möhring, T. Srokowski and D.E. Cross, Nucl. Phys. A533, 333 (1991). 
[16] $k_{\text {eff }}$ refers to the fact that the experimental fold have been increased by few units for those chan nels in which the observed $\gamma$-ray transition do not feed the ground-state. This should account for the unobserved $\gamma$-ray transitions deexciting these excited states.

[17] Z. Majka et al., Phys. Rev. Lett. 58, 322 (1987).

[18] N.G. Nicolis et al., Phys. Rev. C41, 2118 (1990).

[19] S. Landowne and c.H. Dasso, Phys. Lett. 138B, 32 (1984).

IIB5. Lifetimes of Subbarrier $\alpha$ Particles by the Atomic Clock Method - D.G. Sarantites, L. Gallamore, R.J. Charity, [WU], J.R. Beene, M.L. Halbert, and R.L. Varner [ORNL].

The study of subbarrier emission of $\alpha$ particles provided us with a tool for investigating deformation effects in highly excited, rapidly rotating nuclei.[1] We pointed out in Ref. 1 that subbarrier $\alpha$ cmission occurs in general from lower excitations (Fig. $7 \mathrm{c}$ in Ref. 1). Consequently, it is conceivable that a substantial fraction of the subbarrier $\alpha$ 's may originate from states close to the yrast line in competition with the statistical $\gamma$ rays. Such emission was predicted by statistical model calculations some 23 years ago.[2] It is possible that irregularities in the level density near the yrast line may enhance this effect. A similar situation may be responsible for the structural effects on the proton spectra that we have observed [3] and have been predicted [4] in the region near ${ }^{82} \mathrm{Sr}$.

The atomic clock method is based on the following idea. For $\alpha$ particle emissions faster than $4 \times 10^{-18} s$ the $x$-rays follow $\alpha$-emission and therefore are associated with the atom of the daughter nucleus ( $\mathrm{Er}$ in the present case). For $\alpha$-particles emitted at times longer than the $\mathrm{x}$-ray lifetime, the $\mathrm{x}$-rays are characteristic of the atom associated with the compound nucleus ( $\mathrm{Yb}$ in this case). The relative intensities of $\mathrm{x}$-rays from $\mathrm{Yb}$ and $\mathrm{Er}$ in coincidence with $\alpha$ 's can thus be used to determine the fraction of the delayed $\alpha$-particles and the corresporiding mean lifetimes.

We had attempted such an experiment 7 years ago using $4 \mathrm{Si}(\Delta E, E)$ telescopes with a total solid angle of $80 \mathrm{msr}$ and 2 p'anar X-ray detectors inserted in the Spin Spectrometer. That experiment was limited severely by statistics and an upper limit of $\leq 20 \%$ was placed for the fraction of delayed $\alpha$ emission for all $\mathrm{E}_{\alpha}$ energies. We used the reaction ${ }^{64} \mathrm{Ni}+{ }^{100} \mathrm{Mo}$ at $250 \mathrm{MeV}$ (a system studied in Ref. 1). For the ${ }^{164} \mathrm{Yb}$ compound system $\tau_{\mathrm{K}}=4 \times 10^{-18} \mathrm{~S}$ at $E_{K}=52 \mathrm{keV}$. According to calculations [5] a $\mathrm{K}$ vacancy yield of $\sim 2 \%$ is expected in the compound nucleus.

Observation of chiracteristic X-rays emitted during sequential proton evaporation from ${ }^{112} \mathrm{Xe}$ compound nuclei was recently reported by Azaiez et al. [6]. They used coincidences with a plastic scintillator $4 \pi$ box, an $x$-ray detector at $0^{\circ}$ to the beam and the "Chateau de Cristal"[7].

With the availability of the Dwarf Ball the statistics could be increased by a factor of 60 if only one $x$-ray cetector is used. About a year ago we performed such an experiment. We employed an $\mathrm{x}$-ray detector at $138^{\circ}$ to the beam and recorded coincidences with the 


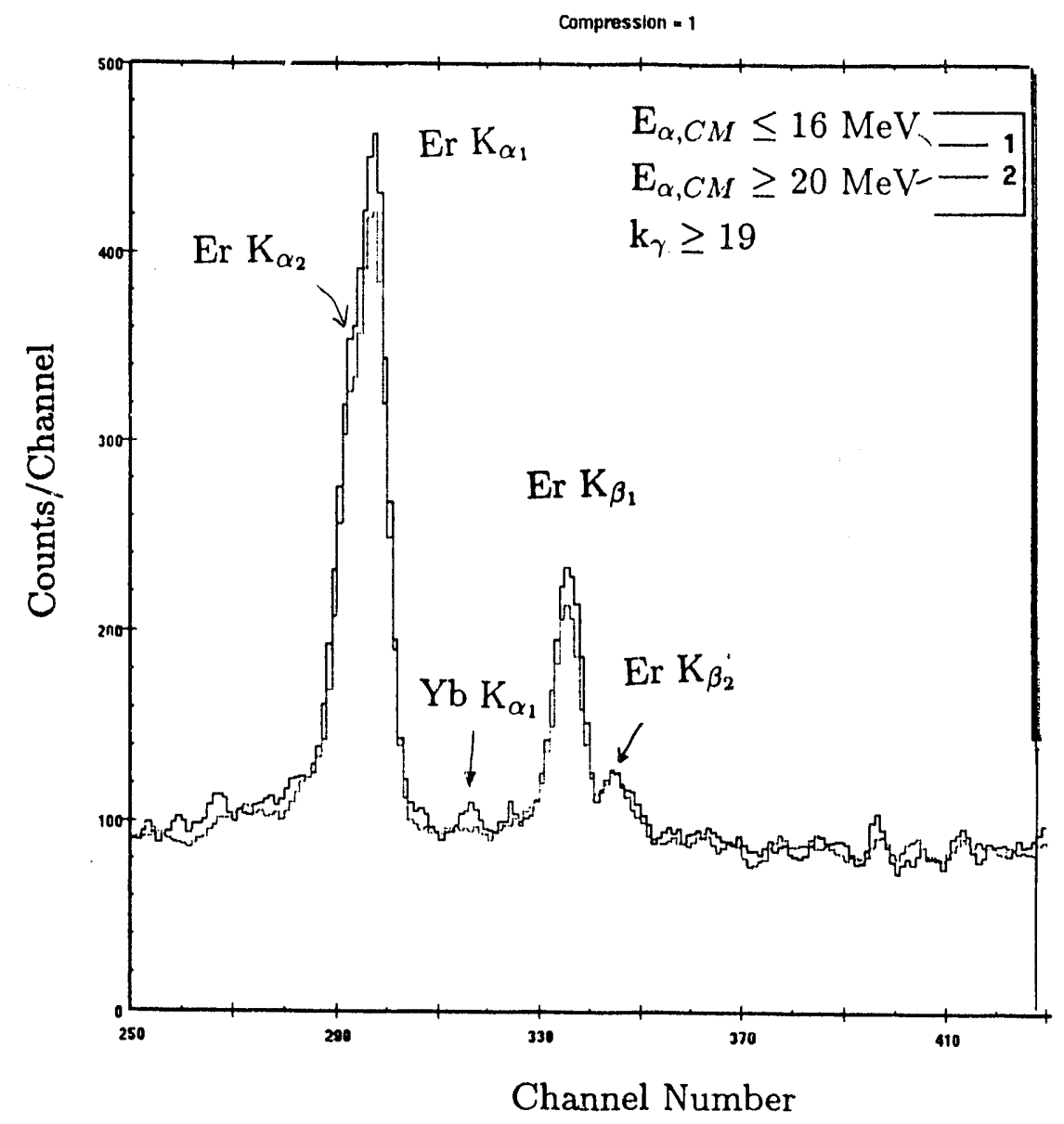

Figure IIB5.1. Spectra of X-rays coincident with $\alpha$-particles with $\mathrm{E}_{\alpha} \leq 16 \mathrm{MeV}$ (thick histogram) and with $\mathrm{E}_{\alpha} \geq 20 \mathrm{MeV}$ (thin histogram). The spectra are gated with $\mathrm{k}_{(\gamma+n)} \geq 19$ from the reaction of $240 \mathrm{MeV}{ }^{60} \mathrm{Ni}+{ }^{100} \mathrm{Mo}$. The two spectra are normalized to the a $60 \mathrm{keV}$ region above $\mathrm{Er}\left(\mathrm{K}_{N_{1}}\right.$ $+\mathrm{K}_{\beta_{2}}$ ) x-rays. The statistically significant peak from the compound nucleus $\mathrm{Yb} \mathrm{K}_{\alpha_{1}}$ is indicated by an arrow.

Dwarf Ball and the Spin Spectrometer. We employed the reaction ${ }^{60} \mathrm{Ni}+{ }^{100} \mathrm{Mo}$ at 240 $\mathrm{MeV}$, selected to maximize the $\left({ }^{60} \mathrm{Ni}, \alpha 2 \mathrm{n}\right)$ channel without appreciable $2 \alpha$ emission. The weak point in this experiment was the X-ray detection. One of the two X-ray detectors from ORNL did not work, and the other was gain unstable at counting rates above 5,000 $\mathrm{c} / \mathrm{sec}$.

Despite these difficulties we collected data for 3.5 days and have found a statistically significant $\mathrm{X}$-ray peak from the $\mathrm{Yb}$ compound nucleus. This was observed when the $\mathrm{X}$-ray spectra were gated by $k_{\gamma} \geq 19$ and had the Dwarf Ball requirement that only $1 \alpha$ particle with an energy $\leq 16 \mathrm{MeV}$ was observed as seen in Fig. IIB5.1 (solid histogram). For comparison the dashed histogram shown is in coincidence with $\alpha$ particles with $\mathrm{E}_{\alpha, C M} \geq 20$ $\mathrm{MeV}$ and does not show the $\mathrm{Yb}_{\alpha_{1}} \mathrm{X}$-ray peak. This small peak ( $60 \pm 20$ counts) is seen only in coincidence with the low energy $\alpha$ particles and has an intensity of $2 \%$ of the Er $\mathrm{K}$ line. The strong $\mathrm{X}$-ray peaks from the $\mathrm{Er}_{\alpha} \mathrm{K}_{\alpha}$ and $\mathrm{K}_{\beta}$ groups seen in Fig. IIB5.1 follow from internal conversion in the de-excitation via $\gamma$ decay. In turn, they provide a means 
of estimating the fraction of the $\alpha$ particles under the present gating conditions that are slower than the X-rays.

One would expect that only $1.0 \%$ of the fusion reaction create a $\mathrm{K}$ vacancy that could lead to an $\mathrm{Yb} \mathrm{K} \mathrm{X}$-ray. On the other hand $\approx 10 \%$ of the cascade will produce an $\mathrm{Er} \mathrm{K}$ $\mathrm{X}$ ray. Therefore $\approx 20 \%$ of the $\alpha$ particles in the low energy gate are emitted on a time scale equal or longer than the $\mathrm{K}$-vacancy filling time.

This is a positive result, that encourages us to proceed to calculate the region of excitation energies and spins in the deexcitation of the compound nucleus that is consistent with the present result.

We plan to undertake further more accurate measurements of this type.

This experiment was done at HHIRF' with assistance from Bob Charity and L. Gallamore (Sobotka'a group). The data analysis was done by L. Gallamore under the supervision of the P.I. The model calculations for the I-vacancy production were done by Lee Sobotka. This work is part of Gallamore's Master's thesis.

[1] N.G. Nicolis et al., Phys. Rev. C41, 2118 (1990).

[2] J.R. Grover, and J. Gilat, Phys. Rev. 157, 802, 814, and 823 (1967).

[3] D.G. Sarantites et al., Phys. Rev. Lett. 64, 2129 (1990).

[4] T. Døssinget al., Nucl. Phys. A287, 137 (1977).

[5] J.V. Andersen et al., Nucl. Instr. and Meth. 192, 79 (1982).

[6] J. Seidel, S. Röhl, R. Lorek, S. Huchler, and M. Dost, Phys. Rev. A32, 2142 (1985).

[7] F. Azaiez et al., Phys. Rev. Lett. 65, 305 (1990)

[8] F. Beck Instrumentation for Heavy Ion Nuclear Research', Ed. D. Shapira (Hardwood Academic, New York, 1985) p. 123.

[9] J.F. Chemin (private communication).

\section{IIB6. Large Deformation in A 170 Nuclei at High Excitation Energies -} M. Thoennessen [MSU], J.R. Beene, F.E. Bertrand, C. Baktash, M.L. Halbert, D.J. Horen, D.C. Hensley, R.L. Varner [ORNL], D.G. Sarantites and D.W. Stracener [WU].

The $\gamma$-decay of the giant-dipole resonance (GDR) built on excited states has been found to be not only a useful tool for study of nuclear shape effects for ruclei as a function of excitation and spin, $[1,2]$ but also a sensitive probe for reaction dynamics. [3,4] For example, the reduction of the fission probability at the early stages of the decay of highly excited compound nuclei was reflected into an enhancement of GDR $\gamma$-rays preceeding fission. $[5,6]$ It was pointed out $[1,2]$ that the GDR does not necessarily measure the minimum of the underlying potential energy surface. It is, however, expected to be sensitive to the average of the deformation of the emitter over the short time scale of the GDR decay. In order to explore these effects we have made a simultaneous study of the GDR decay originating from a composite system when it undergoes fission or when it leads to fusion evaporation residues (ER).

In this work we measured GDR photons in coincidence with ER and with fission fragments for the reactions of $160 \mathrm{MeV}{ }^{16} \mathrm{O}+{ }^{159} \mathrm{~Tb}$, leading to ${ }^{175} \mathrm{Ta}$ at $123 \mathrm{MeV}$ of excitation. 
We have found that the GDR spectrum coincident with ER is well represented by a standard calculation with small deformation. The shape and magnitude of the GDR spectrum in coincidence with the fission fragments cannot be reproduced by calculations with standard parameters of the post-fission and pre-fission contributions to the GDR. Even when a delay for fission is introduced via a friction coefficient $(\gamma \sim 10)$ the shape of the observed GDR is not well reproduced by normal deformation. One must introduce a splitting in the GDR that would correspond to a $\beta \simeq 0.55$. Such a large deformation indicates that the observed GDR spectrum is at least sampling on the average the shape of the nucleus on the way to fission. A similar study of the GDR for fission of neutron deficient $\mathrm{Pb}$ isotopes with comparable fissility to this system produces GDR spectra with much smaller deformations $(\beta=0.38)$. [6] Consequently it may be argued the the present experiment may be sensitive to additional structure effects the that persists at these moderate temperatures.

This work was published as a Physics Letter. A reprint is included as Appendix I.

This experiment was done at HHIRF in collaboration with the ORNL group. The data analysis and interpretation was done M. Thoennessen, now at MSU.

[1] J.H. Gundlach, K.A. Snover, J.A. Behr, C.A. Gosset, M. Kincinska-llabior, and K.T. Lesco, Phys. Rev. Lett. 65, 2523 (1990), and references therin.

[2] Alhassid, Phys. Rev. Lett. 65,2?? (1990)

[3] R. Butsch, D.J. Hofman, C.P. Montoya, P. Paul, and M. Thoennessen, submitted to Phys. Rev. C

[4] M. Thoennessen et. al., in "Nuclear Structure and Heavy Ion Dynamics 1990", Edited by R.R. Betts and J.J. Kolata, Institute of Physics Conference Series 109, Adam Ililger, 1991.

[5] M. Thoennessen, D.R. Chakrabarty, M.G. Herman, R. Butsch, and P. Paul, Phys. Rev. Lett 59, 2860 (1987).

[6] R. Butsch, M. Thoennessen, D.R. Chakrabarty, M.G. Herman, and P. Paul, Phys. Rev. C41, 1530 (1990). 


\section{IIC. PRODUCTION AND STUDY OF HOT NUCLEI}

\section{IIC1. The Statistical Model Evaporation Code EVAP.}

Statistical model calculations have become an indispensable tool for the interpretation of a wide variety of experimental data concerning the decay properties of highly excited compound nuclei. For the surpose of making a realistic description of these properties, we developed the Monte-Cas lo statistical model evaporation code EVAP [1]. Important features of the code include:

(a) The extended excitation energy range of validity, which makes possible calculations of highly excited systems eg. up to $\mathrm{E}^{*} \sim 500 \mathrm{MeV}$.

(b) The consideration of decay channels involving n, p, d, t, $\alpha,{ }^{3} \mathrm{He}$ and ${ }^{6} \mathrm{Li}$ emission.

(c) The use of externally created transmission coefficients $\left(T_{\ell}\right)$, calculated for each individual nucleus and type of emitted particle.

(d) The ability of simulating decays from deformed emitters.

(e) The choice between the Lang or the Gilbert and Cameron level density formalism. The effect of a temperature-dependent level density constant has also been tested (See the following section IIC2).

The usefulness of the above features has been highlighted in the studies of Refs. 2 and 3 concerning particle emission from deformed emitters and the statistical decay of deuterons and tritons from highly excited compound nuclei, respectively.

In most of the current applications we consider emissions of $n, p, \alpha, d, t,{ }^{3} \mathrm{He}$ and ${ }^{6} \mathrm{Li}$. Current efforts include the extension of the code for the description of intermediate mass fragment emission and the population of particle bound excited states in the emitted fragments.

Test calculations are being performed involving 14 "particle" decay channels: $\mathrm{n}, \mathrm{p}, \alpha$, $\mathrm{d}, \mathrm{t},{ }^{3} \mathrm{He},{ }^{6} \mathrm{Li},{ }^{9} \mathrm{Be},{ }^{11} \mathrm{~B},{ }^{12} \mathrm{C},{ }^{14} \mathrm{~N},{ }^{16} \mathrm{O},{ }^{19} \mathrm{~F}$ and ${ }^{20} \mathrm{Ne}$. These calculations employ optical model transmission coefficients $\left(T_{\ell}\right)$ for the first 7 particle decay modes and parabolic model $T_{\ell}$ 's for all the subsequent modes. The parabolic model treatment will be replaced by a more realistic method in the later stages of the development.

Comparisons of the results with existing experimental data as well as the predictions of binary fragmentation models will be made in the following sections.

This work was done exclusively by N. Nicolis (this grant), with useful discussions with the nuclear chemistry group.

[1] Code EVAP, by N.G. Nicolis, D.G. Sarantites and J.R. Beene (unpublished); A description of the code has been given in the 1990-91 Progress Report (COO-40406-7).

[2] N.G. Nicolis, D.G. Sarantites, L.A. Adler, F.A. Dilmanian, K. Honkanen, Z. Majka, L.G. Sobotka, Z. Li, T.M. Semkow, J.R. Beene, M.L. Halbert, D.C. Hensley, J.B. Natowitz, R.P. Schmitt, D. Fabris, G. Nebbia and G. Mouchaty. Phys. Rev. C 41, 2118(1990).

[3] N.G. Nicolis, D.G. Sarantites, L.G. Sobotka and R.J. Charity, Phys. Rev. C 45, 2393(1992). 


\section{IIC2. Statistical Emission of Deuterons and Tritons from Highly Excited Com- pound Nuclei. - N.G. Nicolis, D.G. Sarantites, L.G. Sobotka, and R.J. Charity.}

We recently completed [1] the interpretation of a recent study concerning the behavior of the nuclear level density constant at high excitation energies as deduced from an analysis of particle evaporation spectra and associated multiplicities [2,3]. The present study gave a solution to the overprediction of the experimental deuteron and triton evaporation yields by the statistical model employing standard parameters [3].

In Refs. 2 and 3, Chbihi et al. presented a study of the evaporation spectra of equilibrium sources produced in the reaction $701 \mathrm{MeV}{ }^{28} \mathrm{Si}+{ }^{100} \mathrm{Mo}$. There we used two different methods to extract the level density constant at high excitation. It was found that in order to reproduce the slopes of the light particle spectra, the level density constant of the excited nuclei must have a value of $\mathrm{A} / 10-\mathrm{A} / 11$ at temperatures of 3.5 to $5.5 \mathrm{MeV}$; a conclusion also reached by other authors [4]. However, statistical model calculations with a reduced level density constant lead to an overprediction (in some cases, by a factor of 2 or more) of the experimental deuteron and triton multiplicities. This is in contrast to the finding that the statistical model provides a satisfactory description of deuteron and triton emission from compound nuclei at low excitations $[1,3]$. It was suggested that a more complicated parameter adjustment may be required to treat the decay of highly excited nuclei.

Figure IIC2.1(a) shows the experimental $\mathrm{n}, \mathrm{p}, \alpha, \mathrm{d}$ and $\mathrm{t}$ multiplicities as a function of the initial excitation energy of the emitting source. The solid lines represent the results of EVAP calculations. These calculations were performed using reaction parameters simulating the experimental conditions [1]. They employ optical model transmission coefficients for particle emission and a temperature-dependent level density parameter $a=A / k(T)$ where

$$
k(T)=k(0) \times \frac{1.4}{1+0.4 \exp \left\{-\left(\frac{T}{3}\right)^{2}\right\}} \mathrm{MeV}
$$

This temperature dependence is based on the parametrization of Ormand et al. [5] ind accounts for an inclusion of thermal and curantal fluctuations on a low temperature level density constant $k(0)$. Choosing $k(0)=8.0$ brings consistency with the level density constants extracted from the analysis of the evaporative spectra of Ref. 3 .

The neutron, proton and $\alpha$-particle multiplicities are well reproduced in the whole excitation range $\left(100 \mathrm{MeV} \leq \mathrm{E}^{*} \leq 405 \mathrm{MeV}\right)$. However, the deuteron and triton multiplicities are systematically overpredicted. This overprediction increases with excitation energy up to more than a factor of 2 . The same trend was also observed in the simplified statistical model treatment of Ref. 3 where energy-independent level density constants were used.

Due to the high excitation energies involved, changes in the critical angular momentum for fusion do not greatly affect the calculated particle emission yields. Also, changes in the level density constant were shown inadequate to account for the observed discrepancies 
(a)
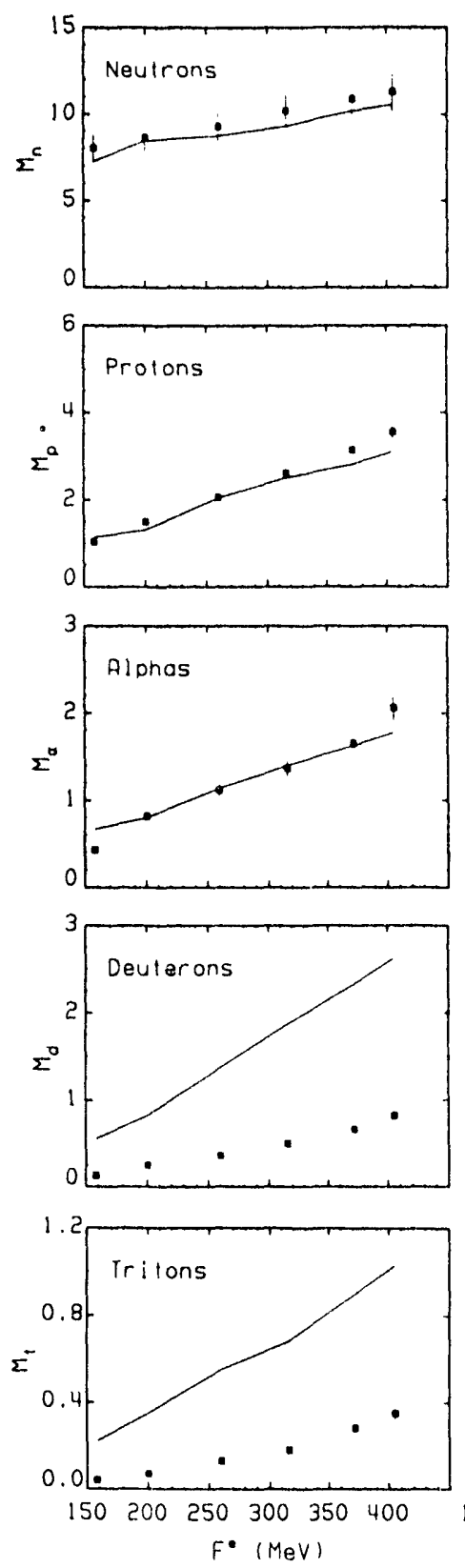

(b)
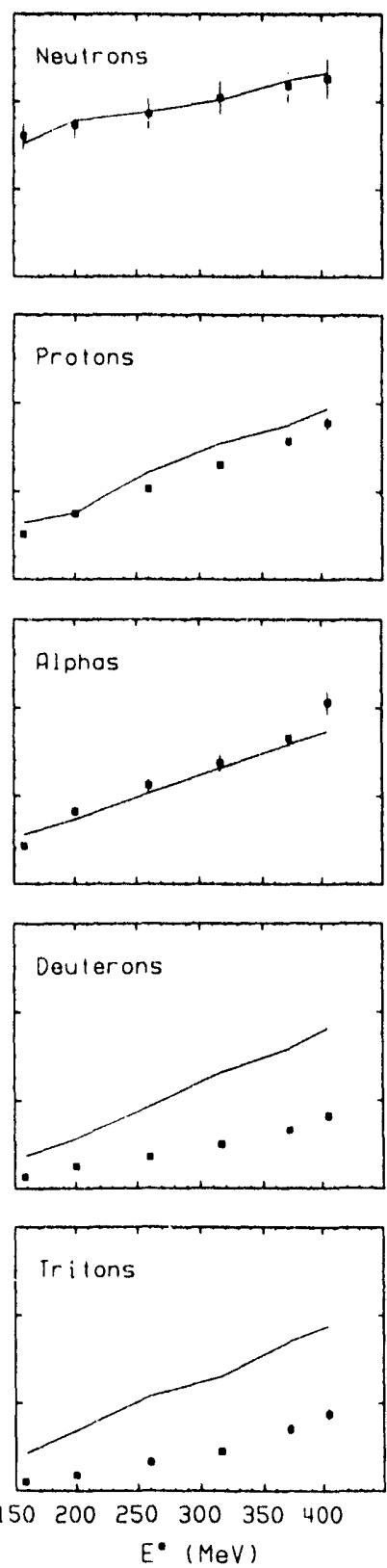

(c)
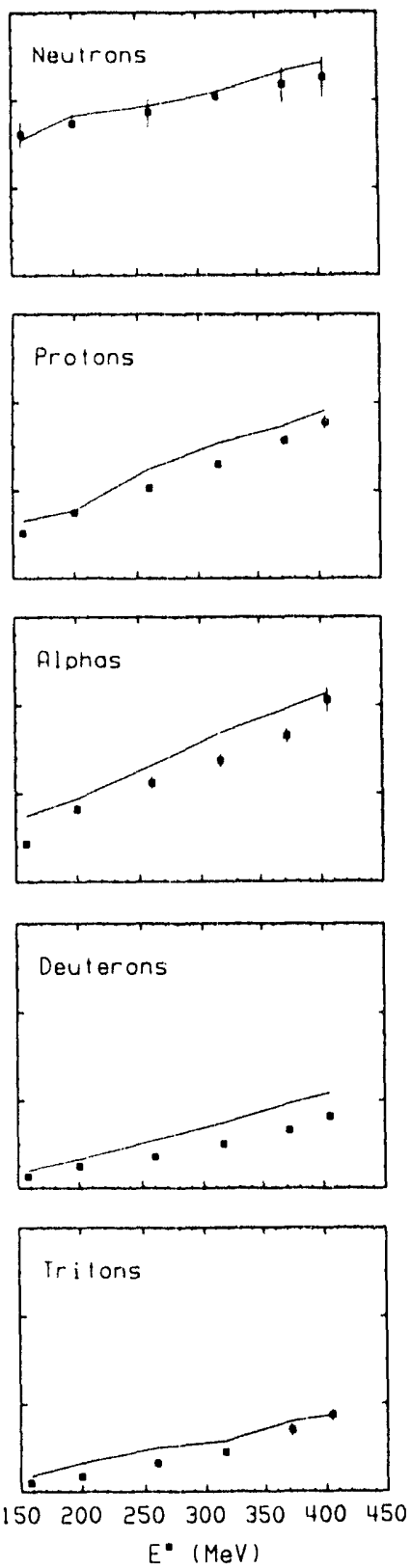

Figure IIC2.1. Experimental $\mathrm{n}, \mathrm{p}, \alpha, \mathrm{d}$ and $\mathrm{t}$ multiplicities (symbols) as a function of the excitation energy of the emitting source in reactions of $701 \mathrm{MeV}{ }^{28} \mathrm{Si}$ on ${ }^{100} \mathrm{Mo}$. The solid lines show the predictions of the statistical model with a temperature-dependent level density constant as described in the text. The three columns of panels show calculations with transmission coefficients: (a) from the optical model, (b) from the IWBC model and (c) from the optical model for $n, p, \alpha$ and the DRAF method for $d$ and $t$ emission.

in the deuteron and triton yields [3]. However, another important factor that determines particle emission is the transmission coefficients. 
In the statistical model description, the penetrability for particle emission is related to the one of the inverse (capture) process through the principle of detailed balance. The traditional method of calculating particle penetrabilities employes $T_{\ell}$ 's derived from an optical model (OM) calculation with standard parameters [6]. For many years, this mothod has been able to describe successfully the decay properties of low-excited compound nuclei for which $\mathrm{n}, \mathrm{p}$ and $\alpha$-emission are the most probable decay modes.

Alexander, Magda and Landowne, recently reviewed the logical basis of using optical model $(\mathrm{OM}) T_{\ell}$ 's in statistical model calculations [7]. A comparison of OM $T_{\ell}$ 's was made with the ones derived from an ingoing-wave boundary condition (IWBC) calculation. These $T_{\ell}$ 's give the transmission probability through the real potential barrier. It was pointed out that processes like transparency, shape resonances or peripheral absorption are normally present in the OM $T_{\ell}$ 's. These processes raise questions on the suitability of the OM $T_{\ell}$ 's in describing the inverse process of capture from an excited nucleus. It was suggested that simple barrier penetration $T_{\ell}$ 's, such as those of the IVBC model, may be more appropriate for use in statistical model calculations.

Following Ref. 7 , we created a transmission coefficient set using the real part of the OM potentials [6] and replaced the role of the imaginary parts with an ingoing-wave boundary condition. The statistical model calculations for the multiplicity data are shown in Fig. IIC2.1(b). The neutron, proton and alpha multiplicity data are again well reproduced but the calculated $\mathrm{d}$ and $\mathrm{t}$ yields are still in excess of the experimental ones. However, the use of IWBC $T_{\ell}$ 's reduces the discrepancies found with the original OM $T_{\ell}$ set. This improvement is attributed to the elimination of processes in the OM absorption which are not related to fusion. Therefore, it becomes desirable to examine transmission coefficient sets describing fusion of deuterons on different targets as candidates for the description of the emission properties of our systems.

In a recent study, West et al. [8] measured excitation functions for the ground and an excited isomer state in the reaction ${ }^{52} \mathrm{Cr}(\mathrm{d}, 2 \mathrm{n}){ }^{52} \mathrm{Mn}{ }^{g}, m$. They found that the assumption of equating the fusion cross section of $\mathrm{d}+{ }^{52} \mathrm{Cr}$ to the OM absorption (reaction) cross section fails to explain the observable cross sections. Subsequently, Mustafa et al. [9], were able to describe the above data using $\sigma_{f u s}$ from the direct-reaction approach to fusion (DRAF) of Udagawa et al. [10]. In the DRAF method, the fusion part of the reaction process is incorporated in the inner region of the imaginary part of the optical potential. The outer part is associated with incomplete fusion or direct reaction processes. This separation is achieved with the introduction of a fusion radius. A decomposition of the OM absorption cross section $\sigma_{a b s}$ into a complete fusion $\sigma_{C F}$ and a direct interaction part $\sigma_{D I}$ is then obtained: $\sigma_{a b s}=\sigma_{C F}+\sigma_{D I}$. The usefulness of the method lies in the fact that once the fusion radius is determined, a description of the complete and direct interaction process can be made on the same footing. Furthermore, it provides us with a method of creating $T_{\ell}$ 's related to the fusion part of the absorption process in the OM description. Using this method, Mastroleo et al. [11] were able to describe the cross sections of various processes 
in $\mathrm{d}+{ }^{93} \mathrm{Nb}$ reactions; a reaction system with a mass similar to our emission systems. The studies of Refs. 9 and 11 show that the direct i.ateraction part is a sizable fraction of the OM absorption cross section in deuteron induced reactions.
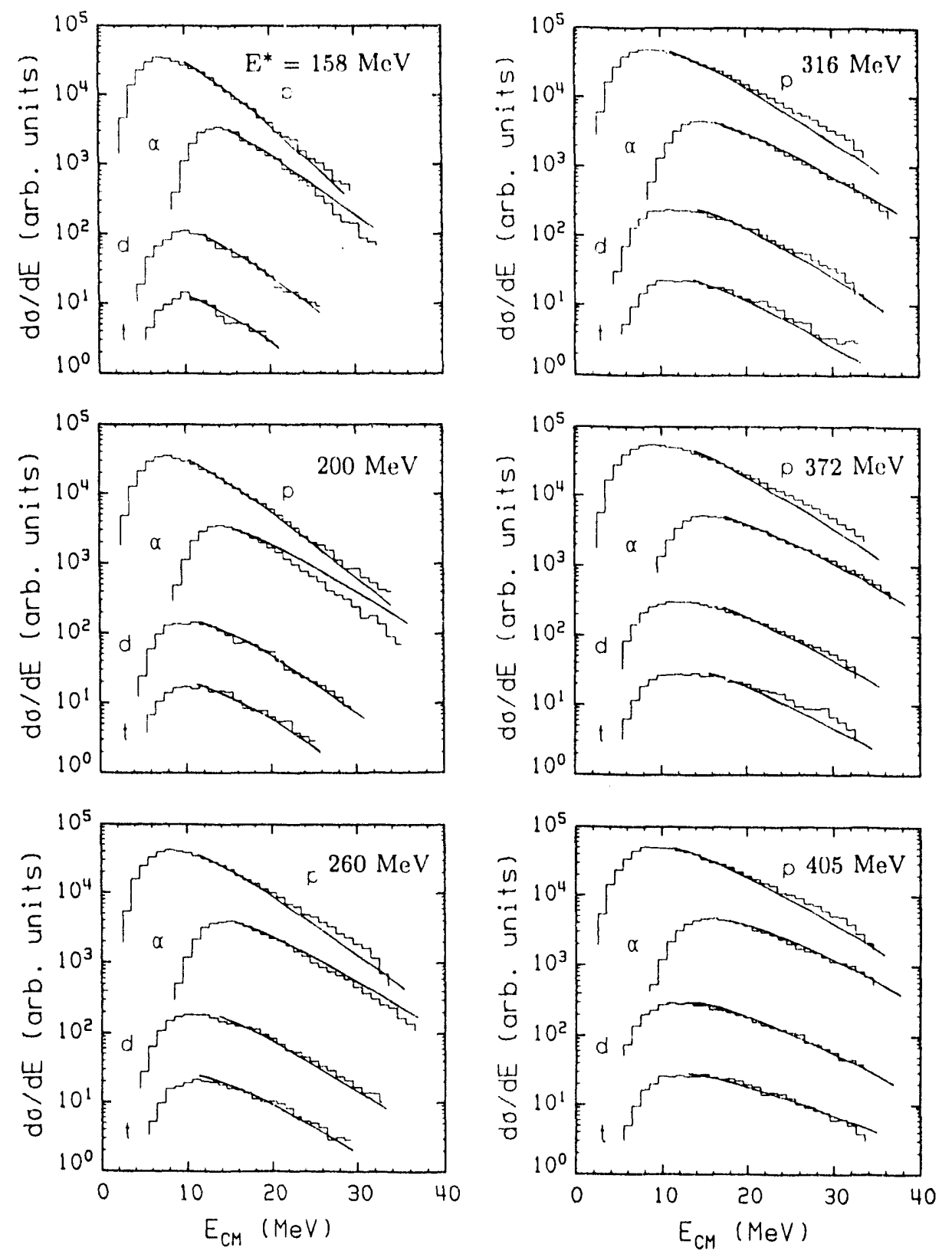

Figure IIC2.2. Comparison between the calculated $\mathrm{p}, \alpha, \mathrm{d}$ and $\mathrm{t}$ center of mass energy spectra (histograms) at the indicated excitation energies of the emitting source. The solid lines represent the experimental slopes obtained from evaporative moving source fits. 
A transmission coefficient set was created with OM $T_{\ell}$ 's for $\mathrm{n}, \mathrm{p}$ and $\alpha$-particles and DRAF $T_{\ell}$ 's for deuterons and tritons. A fusion radius for deuterons consistent with that of Ref. 11 and a radius of a similar magnitude for tritons was used. The corresponding statistical model calculations are compared with the data in Fig. IIC2.1(c). The calculation provides a good agreement of the experimental data in the whole excitation energy range. The small overprediction of $M_{\alpha}$ is not considered significant, given that the uncertainty in the atomic numbers of our emitting systems is \pm 1 .

The same calculation produces particle energy spectra whose slopes are consistent with the experimental ones. In Fig. IIC2.2, the histograms show the calculated proton, alpha, deuteron and triton energy spectra in the center of mass system. The spectra have been displaced on the vertical axis for pictorial reasons. The initial excitation energies of the emitting sources are shown in the figure. The solid lines represent the slopes of the evaporative source fits to the data [3]. Our approach of using a temperature-dependent level density constant together with the introduction of DRAF $T_{\ell}$ 's for denterons and tritons produces a simultaneous description of the particle multiplicities and the slopes of the evaporation spectra.

Our analysis shows that the statistical model with optical model transmission coefficients for $\mathrm{n}, \mathrm{p}, \alpha, \mathrm{d}$ and $\mathrm{t}$ emission systematically overpredicts the denteron and triton emission yields at high excitation energies ( $E^{*}$ up to $405 \mathrm{MeV}$ ). These discrepancies are attributed to absorption processes in the optical model inverse cross section which are not related to fusion. It seems that such processes represent a small fraction of the OM absorption cross section for neutrons, protons and alphas. This explains the success of the OM $T_{\ell}$ 's in statistical model calculations where $n, p$ and $\alpha$-emissions dominate. However, the optical model treatment breaks down for deuterons and tritons whose emission becomes more important at high excitation energies.

The present work demonstrates that the elimination of the direct interaction components from the OM absorption is necessary for a successful description of denteron and triton emission in statistical model calculations. The DRAF method provides a quantitative means of isolating these processes and extracting a transmission coefficient set consistent with the fusion process. With this method, it became possible to reproduce the deuteron and triton emission yields from highly excited compound nuclei; a result which could not be achieved with any other statistical model parameter change. For deuteron emission in particular, the employed fusion radius is consistent with a deuteron fusion cross section analysis in the mass region of the present study. A global description of particle emission in $701 \mathrm{MeV}$ reactions of ${ }^{28} \mathrm{Si}$ on ${ }^{100} \mathrm{Mo}$ reactions was given in the context of a temperature-dependent level density constant.

We believe that systematic studies of deuteron and triton induced reactions are needed in order to extract and systematize the fusion radii. This may provide valuable information for parameters to be used in the description of statistical deuteron and triton emission from highly excited nuclei. 
This work was done exclusively by $\mathrm{N}$. Nicolis (this grant), with useful discussions with the nuclear chemistry group, and was published in Phys. Rev. (see Appendix M for a reprint).

(1) N.G. Nicolis, D.G. Sarantites, L.G. Sobotka and R.J Charity, Phys. Rev. ('45, 2393 (1992).

[2] A. Chbihi, L.G. Sobotka, Z. Majka, D.G Sarantites, D.W Stracener, V Abenante, T.M. Semkow, N.G. Nicolis, D.C. Hensley, J.R. Beene and M L. Halbert, Phys. Rev. C43, 65\%2 (1991).

[3] A. Chbihi, L.G. Sobotka, N.G. Nicolis, D.G. Sarantites, D.W. Stracener, Z. Majka, D.C. Hensley, J.R. Beene and M.L. Halbert, Phys. Re $\quad(43,666$ (1991).

[4] S. Shlomo and J.B. Natowitz, Phys. Lett. B252, 187 (1990); and references therein.

[5] W.E. Ormand, P.F. Bortignon, A. Bracco and R.A. Broglia, Phys. Rev. (40, 1510 (1989).

[6] C.M. Perey and F.G. Perey, At. Nucl. Data Tables 17, 1 (1976).

[7] J.M. Alexander, M.T. Magda and S. Landowne, Phys. Rev. C42, 1092 (1991).

[8] H.I. West, Jr., R.G. Lanier and M.G. Mustafa, Phys. Rev. C35, 2067 (1987).

[9] M.G. Mustafa, T. Tamura and T. Udagawa, Phys. Rev. C35, 2077 (1987).

[10] T. Udagawa, B.T. Kim and T. Tamura, Phys. Rev. C32, 124 (1985).

[11] R.C. Mastroleo, T. Udagawa and M.G. Mustafa, Phys. Rev. C42, 683 (1990).

\section{IIC3. Heavy-Fragment Emission as a probe of the Thermal Properties of Highly Excited Compound Nuclei - N.G. Nicolis and D.G. Sarantites}

The Monte-Carlo nature of the code EVAP makes possible the study of a number of correlations between experimental and/or theoretical quantities thus providing insight into the evaporation process.

An example of a high energy deexcitation calculation is given in Fig. IIC3.1 for the reaction $441 \mathrm{MeV}{ }^{19} \mathrm{~F}+{ }^{100} \mathrm{Mo} \longrightarrow{ }^{119} \mathrm{Sb}^{*}(373 \mathrm{MeV})$. The figure shows contour plots on the excitation energy $\left(E^{*}\right)$ versus angular momentum plane of the neutron, proton, alpha and deuteron emitting states. The numbers on the contours give the intensity of each level in arbitrary units. The corresponding particle multiplicities are also given. The calculation was performed with the parameters mentioned in Section IIC2; i.e. DRAF $T_{\ell}$ 's for deuterons and tritons and optical model $T_{\ell}$ 's for the rest of the particles. The temperature-dependent level density constant of Ormand et al. was also used.

The contour plots show that for neutron, proton and alpha emission low excitation states are more frequently sampled. The angular momentum dependence is stronger for alpha than it is for proton or neutron emission. This is a rather well known fact. However, the emission pattern for deuterons is different. Deuterons are emitted primarily from very high excitation states and have a weak angular momentum dependence. Emission of other energetically expensive modes like ${ }^{3} \mathrm{He}, \mathrm{t}$ and ${ }^{6} \mathrm{Li}$ shows a similar pattern.

A consequence of this difference is reflected in the contribution of different $\left(E^{*}, I\right)$ emitting states to the total particle emission spectrum for each decay mode. The thick lines of Figs. IIC 3.2 and IIC 3.3 show the calculated total center of mass spectra for $n, p$, $\alpha, \mathrm{d}, \mathrm{t},{ }^{3} \mathrm{He}$ and ${ }^{6} \mathrm{Li}$ emission. We have examined the contribution to these spectra from emitting states at different excitation energies by constructing the partial spectra gated by the excitation energy within the following $\mathrm{E}^{*}$-windows: $0-100 \mathrm{MeV}$ (curve 4), 100-200MeV 
(curve 3), $200-300 \mathrm{MeV}$ (curve 2), and $\mathrm{E}^{*} \geq 300 \mathrm{MeV}$ (curve 1). In all cases, the partial spectra corresponding to the highest excitation energies are the hardest. They correspond to energetic non-stretched transitions which cool off the emitting nuclei. The closeness of the slopes of the curves 1 and 2 to the ones of the total spectra makes the total spectral slopes useful in the determination of the level density of highly excited nuclei. However, statistical correction factors are needed in the analysis of the evaporation spectra as was done in Ref. 1.
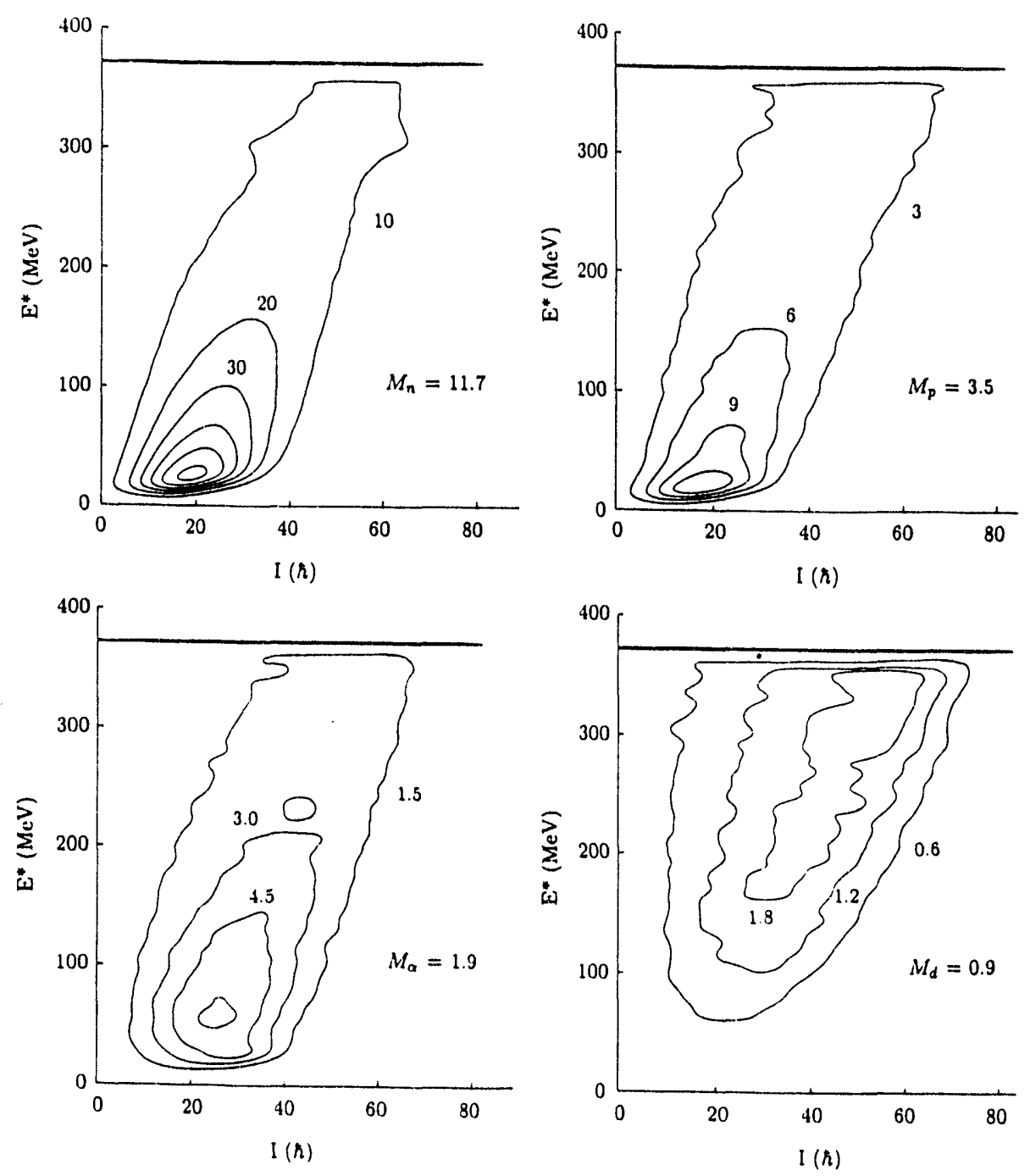

Figure IIC3.1. Calculated $\left(\mathrm{E}^{*}, \mathrm{I}\right)$ distributions of the neutron, proton, $\alpha$, and deuteron emitting states in the reaction $441 \mathrm{MeV}{ }^{19} \mathrm{~F}+{ }^{100} \mathrm{Mo} \longrightarrow{ }^{119} \mathrm{Sb}^{*}(373 \mathrm{MeV})$. 

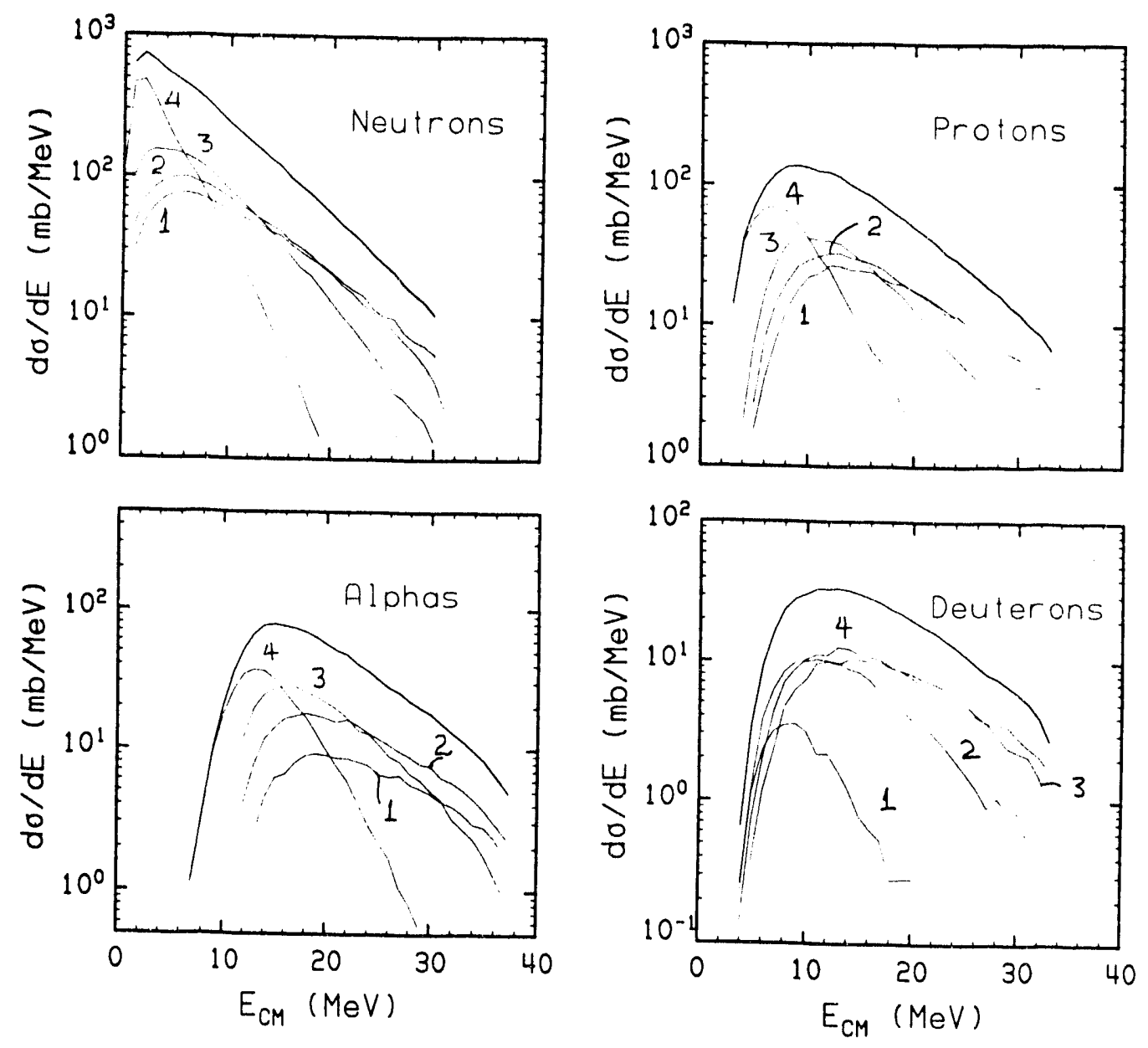

Figure IIC3.2 Calculated n, p, $\alpha$ and d CM spectra in the reaction $441 \mathrm{MeV}{ }^{19} \mathrm{~F}+{ }^{100} \mathrm{Mo} \longrightarrow$ ${ }^{119} \mathrm{Sb}^{*}(373 \mathrm{MeV})$ (Thick lines). The numbered thin lines show the contributions to these spectra from emitting states in the excitation energy ranges given in the text.

Of interest are the low energy parts of the partial spectra compared to the total ones. Proton and alpha emission show a common behavior. The low energy parts of the total spectra are dominated by emissions originating from low-excitation energy states. These are stretched emissions that occur close to the yrast lines. This fact for alpha emission was brought up in the study of Ref. 2 where it was pointed out that low energy alpha emission probes the emission properties of nuclei at a finite but low excitation. One may conclude that neutron, proton and alpha spectra are not useful if we want to obtain information concerning the barrier properties of highly excited nuclei.

However, Figs. IIC3.2 and IIC 3.3 show that the corresponding spectra for the rare (energetically costly) decay modes of $\mathrm{d}, \mathrm{t},{ }^{3} \mathrm{He}$ and ${ }^{6} \mathrm{Li}$ emission, are almost free of low $\mathrm{E}^{*}$ emissions. This is a consequence of the fact that all of these emissions take place far from the yrast line. 

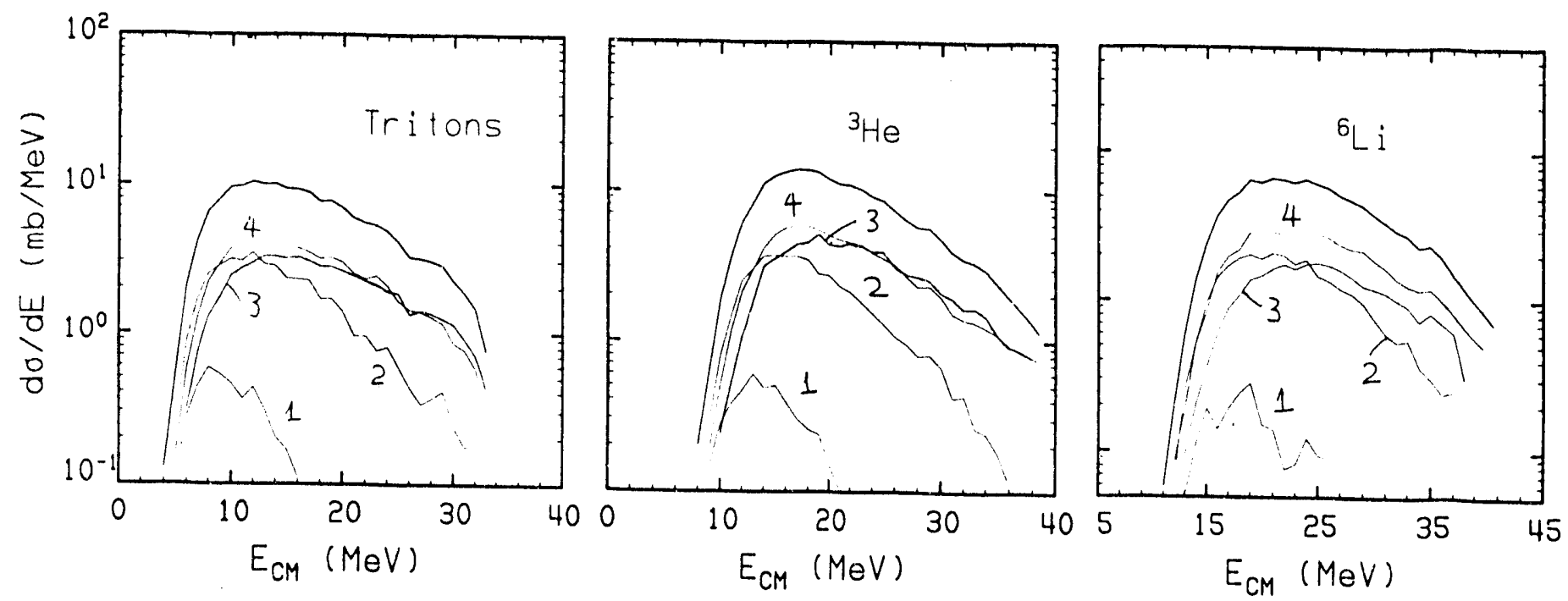

Fig. IIC3.3 Same as Fig. IIC3.2, for tritons, ${ }^{3} \mathrm{He}$ and ${ }^{6} \mathrm{Li}$.

Fleckner, Sauer and Mosel [3] have studied the excitation energy dependence of Coulomb- barriers in a folding model calculation using thermal Hartree Fock nuclear densities. For alpha emission from ${ }^{208} \mathrm{~Pb}\left(\mathrm{E}^{*} \sim 500 \mathrm{MeV}\right.$ or $\left.\mathrm{T}=5 \mathrm{MeV}\right)$ the barrier has been predicted to be lower by $0.7 \mathrm{MeV}$. At the same excitation, however, the barrier for ${ }^{12} \mathrm{C}$ emission is lowered by $1.7 \mathrm{MeV}$.

Our preliminary results indicate that a temperature effect in the barrier reduction for alpha emission would be unnoticed in the total spectrum. However, the barrier reduction in the evaporation spectra of heavier fragments such as ${ }^{12} \mathrm{C}$ could be pronounced.

The upgrading of EVAP to include emission of heavy fragments may give us quantitative estimates of such effects in order to establish the use of heavy fragment emission spectra as probe of the evaporation barrier of highly excited compound nuclear systems.

This work was done exclusively by N. Nicolis (this grant), with useful discussions with the nuclear chemistry group.

[1] A. Chbihi, L.G. Sobotka, N.G. Nicolis, D.G. Sarantites, D.W. Stracener, Z. Majka, D.C. Hensley, J.R. Beene and M.L. Halbert. Phys. Rev. C 43, 666(1991).

[2] N.G. Nicolis, D.G. Sarantites, L.A. Adler, F.A. Dilmanian, K. Honkanen, Z. Majka, L G. Sobotka, Z. Li, T.M. Semkow, J.R. Beene, M.L. Halbert, D.C. Hensley, J.B. Natowitz, R.P. Schmitt, D. Fabris, G. Nebbia and G. Mouchaty. Plyss. Rev. C 41, 2118(1990).

[3] J. Fleckner, G. Sauer and U. Mosel, Phys. Lett. 65B, 316(1976).

\section{IIC4. On the Use of IWBC Transmission Coefficients in the Statistical Model:} Implications in the Particle Evaporation Spectra - N.G. Nicolis and D.G. Sarantites

In the statistical model, the decay of an excited nucleus is determined by two factors: the level density of the populated nuclei and the appropriate transmission coefficients. The transmission coefficients for particle emission are related through the principle of detailed 
balance, to the one of the inverse process, namely, the capture of the particle by the excited daughter nucleus [1,2]. A commonly used set of transmission coefficients is the one describing the absorption of the incident particle by the target in the optical model [2].

Alexander, Magda and Landowne [3] recently reviewed the logical basis of using optical model (OM) transmission coefficients $\left(T_{\ell}\right)$ in statistical model calculations. A comparison of the OM $T_{\ell}$ 's was made with the ones derived from an ingoing-wave boundary condition (IWBC) calculation [4]. The latter $T_{\ell}$ 's give the transmission probability through the real part of the optical model potential. The role of the imaginary potential is replaced by the ingoing-wave boundary condition. It was pointed out that processes like transparency, shape resonances or peripheral absorption are normally present in the OM $T_{\ell}$ 's. Such processes should not be included in a model describing the absorption of a particle by a nucleus, if one wishes to describe the process of particle emission with the same set of $T_{\ell}$ 's. Alexander et al. [3] suggested that $T_{\ell}$ 's arising from a strong absorption model such as the IWBC model may be more appropriate for use in statistical model calculations.

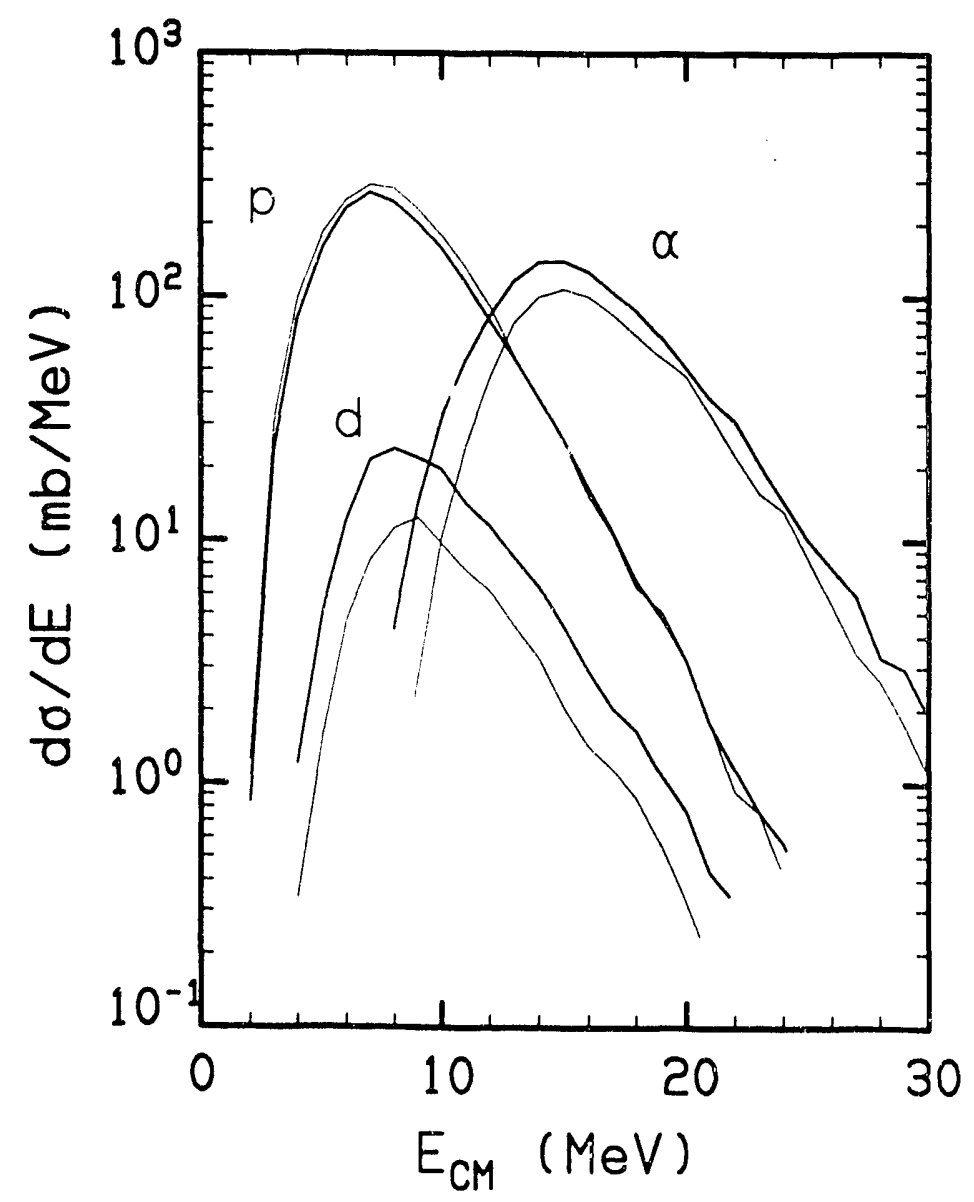

Fig. IIC4.1. Calculated center of mass proton, alpha and deuteron spectra with optical model (thick lines) and IWBC transmission coefficients (thin lines). 
In our study of deuteron and triton emission from highly excited nuclei [5] we showed that the statistical model with $\mathrm{OM} T_{\ell}$ 's describes satisfactorily the $\mathrm{p}, \mathrm{d}, \mathrm{t}, \alpha$ emission spectra and cross sections in the reaction: $121 \mathrm{MeV}{ }^{14} \mathrm{~N}+{ }^{103} \mathrm{Rh} \longrightarrow{ }^{117} \mathrm{Te}^{*}(107 \mathrm{MeV})$. The effect of IWBC $T_{\ell}$ 's in the calculated particle multiplicities was tested at high excitation energies. In this Section, we make remarks concerning the detailed shape of the calculated energy spectra of the emitted particles using OM and IWBC $T_{\ell}$ 's.

In Fig. IIC4.1, we compare the calculated center of mass proton, alpha and deuteron spectra with OM $T_{\ell}$ 's (thick lines) and IWBC $T_{\ell}$ 's created according to Ref. 3 (thin lines). In both cases, the slopes of the particle spectra seem to be similar for all particle types. Indeed, the slopes of the evaporation spectra are determined mainly by the level densities, since the transmission coefficients approach a limiting value for high channel energies in both models. In the low energy region, no significant differences are seen in the spectral shapes for protons and deuterons. However for alpha particle emission, the low energy part of the spectrum calculated with IWBC $T_{\ell}$ 's shows a shift of $\sim 1 \mathrm{MeV}$ up in energy, compared to that obtained with $\mathrm{OM} T_{\ell}$ 's.

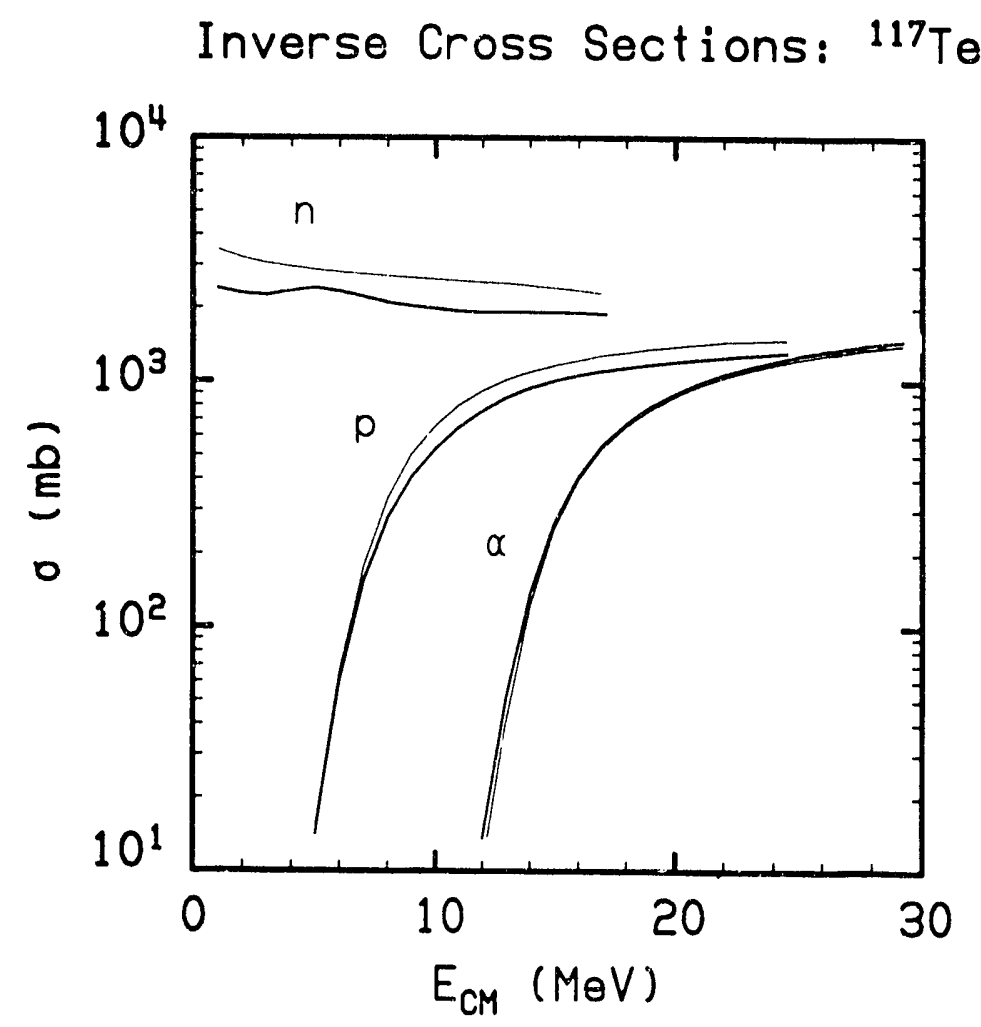

Figure IIC4.2. Calculated inverse cross sections for neutrons, protons and alpha particles for ${ }^{117} \mathrm{Te}$. The thick lines correspond to the optical and the thin lines to the IWBC model. (thin lines). 
The behavior of the low energy alpha emission is not related to a difference in the evaporation barriers for alpha emission. We have verified that the OM and IWBC barriers are similar for partial waves up to $\ell=10 \hbar$. A detailed analysis of the events comprising the low energy spectra of Fig. IIC4.1 shows that the suppression of the low energy alpha emission in the IWBC model is due to: a) The suppression of high- $\ell$ partial waves for alpha emission, and b) The enhancement of low- $\ell$ partial waves for neutron and proton emission which enhances their competition with alpha emission at low excitation energies. These trends in the transmission coefficients have been shown in Ref. 3 .

In a number of studies, the behavior of the low-energy particle spectra has been used in the interpretation of the properties of the emitting system. Therefore, the above difference in the calculated alpha energy spectra by the two models deserves further investigation.

In Fig. IIC4.2 we show the neutron, proton and alpha inverse cross sections that lead to ${ }^{117} \mathrm{Te}$. The thick lines correspond to the predictions of the optical and the thin ones to the IWBC model. For neutrons, the IWBC model overestimates the OM inverse cross section. Similarly, the proton inverse cross section is overestimated at high energies. The $\alpha$ inverse cross section is underestimated at all energies. 1 t the effect is more significant at low energies.

Our OM calculations for neutrons have been compared and found consistent with the data of Ref. 6 as far as the total and absorption cross sections are concerned. It has to be noted that the optical model parameters of Ref. 6 have been used excessively in the past in Hauser-Feshbach calculations. Therefore, the excess of neutron cross sections predicted by the IWBC model cannot be justified in terms of the existing data. For protons, comparisons with excitation functions of proton induced reactions has to be made. The underprediction of the optical model cross section by the IWBC model is discussed further in Section D.

This work was done exclusively by N. Nicolis (this grant), with useful discussions with the nuclear chemistry group.

[1] T. Ericson, Adv. Phys. 9, 425(1960).

[2] H. Feshbach, C.E. Porter and V.F. Weisskopf, Phys. Rev. 90, 166(1953).

[3] J.M. Alexander, M.T. Magda and S. Landowne, Phys. Rev. C 42, 1092(1991).

[4] G.H. Rawitscher, Nucl. Phys. 85, 337(1966).

[5] N.G. Nicolis, D.G. Sarantites, L.G. Sobotka and R.J. Charity, Phys. Rev. C 45, 2393(1992).

[6] F. Bjorklund and S. Fernbach, Phys. Rev. 109, 1295(1958).

IIC5. A Study of Transparency in the Optical Model. - N.G. Nicolis and D.G. Sarantites.

A comparison was made in Section IIC4 of the inverse alpha cross sections for ${ }^{117} \mathrm{Te}$ using optical model and IWBC transmission coefficients according to the prescription given in Ref. 1. These inverse cross sections are compared in Table E1. We see that $\sigma(I W B C)$ 
underestimates $\sigma(O M)$ by a percentage that increases as the alpha particle energy decreases.

The introduction of the IWBC model was made in order to eliminate processes like transparency, shape resonances or peripheral absorption which are expected to be present in the OM $T_{\ell}$ 's. The interaction of alpha particles on different targets is usually well described with strong absorption models. One expects the optical model transparency to become stronger as the alpha particle energy increases. This is in contradiction with the trend between $\sigma(I W B C)$ and $\sigma(O M)$ seen in Table 1.

Table IIC5.1. Alpha-particle inverse cross-sections for ${ }^{117} \mathrm{Te}$.

\begin{tabular}{lrrr}
\hline$E_{C M}(\mathrm{MeV})$ & $\sigma_{O M}(\mathrm{mb})$ & $\sigma_{I W B C}(\mathrm{mb})$ & $\Delta(\%)$ \\
\hline 8.0 & 0.0013 & 0.0004 & -69.2 \\
9.0 & 0.0279 & 0.0101 & -63.8 \\
10.0 & 0.3332 & 0.1537 & -53.9 \\
11.0 & 2.5539 & 1.4491 & -43.3 \\
12.0 & 13.6027 & 9.2757 & -31.8 \\
13.0 & 51.6325 & 41.2538 & -20.1 \\
14.0 & 137.8610 & 123.2652 & -10.6 \\
15.0 & 266.6298 & 252.0102 & -5.5 \\
16.0 & 410.2834 & 395.8602 & -3.5 \\
17.0 & 549.3282 & 533.5934 & -2.9 \\
18.0 & 676.7311 & 658.5908 & -2.7 \\
19.0 & 791.3835 & 770.2637 & -2.7 \\
20.0 & 894.1394 & 869.7432 & -2.7 \\
21.0 & 986.3234 & 958.4808 & -2.8 \\
22.0 & 1069.2574 & 1037.8679 & -2.9 \\
23.0 & 1144.1245 & 1109.1167 & -3.1 \\
24.0 & 1211.9523 & 1173.2772 & -3.2 \\
25.0 & 1273.6075 & 1231.2491 & -3.3 \\
26.0 & 1329.8412 & 1283.7876 & -3.5 \\
27.0 & 1381.2903 & 1331.5913 & -3.6 \\
28.0 & 1428.4835 & 1375.1753 & -3.7 \\
29.0 & 1471.8898 & 1415.1500 & -3.9 \\
30.0 & 1511.9094 & 1451.8452 & -4.0 \\
\hline
\end{tabular}

We have undertaken a study of the optical model transparency by calculating the quantum flux and divergence of alpha particles incident on different targets. Below, we describe briefly our method which we applied first in the case of alpha particle scattering 
on ${ }^{42} \mathrm{Ca}$ due to the detailed studies done in this system [4]. The starting point is the Schrödinger equation which for the motion of particles in a complex potential $\mathrm{U}=\mathrm{V}+\mathrm{iW}$, with $\mathrm{W}<0$ is equivalent to the continuity equation with a sink term, i.e.

$$
\frac{\partial}{\partial t}\left(\Psi^{*} \Psi\right)+\nabla \cdot \vec{J}=\frac{2}{\hbar} \Psi^{*} W \Psi
$$

here $\vec{J}$ is the probability current dusity vector and $\Psi$ is the (OM) wave function of the particle. The sink term represents the loss of particles per unit volume per unit time. Assuming a steady state and integrating Eq. 1 in a sufficiently large volume $V$ of radius $\mathrm{R}$ (enclosing the nucleus) we get

$$
\int_{S} J_{\rho} d S=2 \pi R^{2} \int_{0}^{\pi} J_{\rho} \sin \theta d \theta=\frac{2}{\hbar} \int_{V} \Psi^{*} W \Psi d V
$$

where we made use of the Stoke's theorem. The second term in Eq. (2) expresses the number of particles lost per unit time; $J_{\rho}$ is the component of the probability current density vector normal to the integration surface $S$. It is negative for the ingoing and positive for the outgoing flux. Therefore, we can determine the radius at which we have reflections by examining the positive and negative contributions to this integral as a function of the distance from the center of the nucleus.

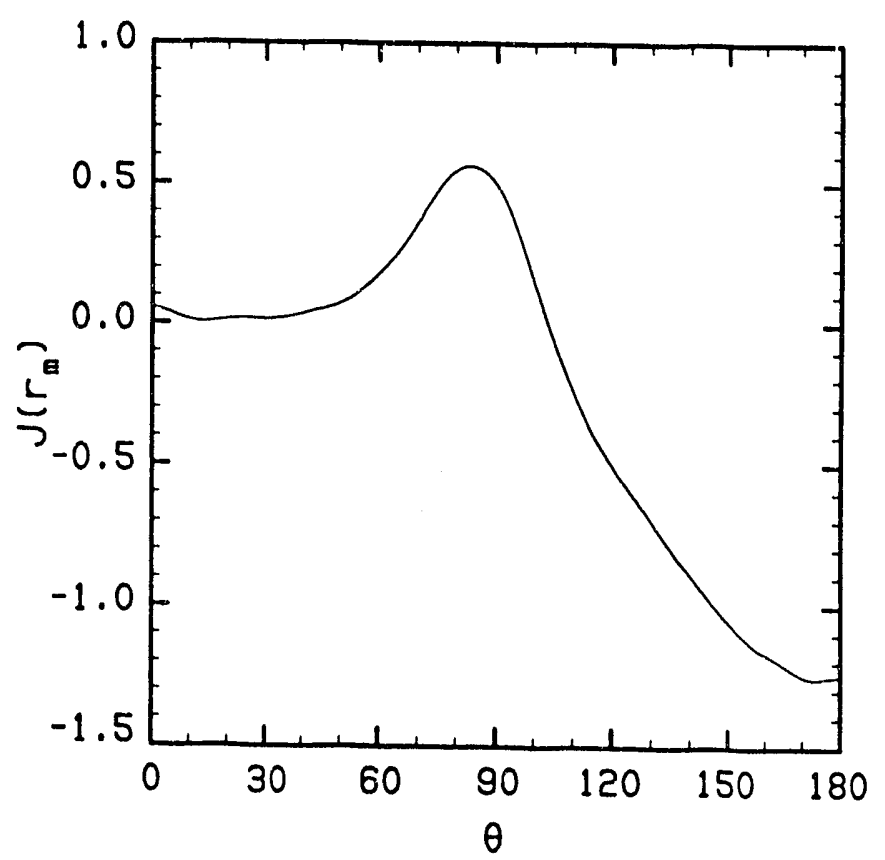

Figure IIC5.1. Radial component of the probability current density vector $J_{\rho}$ for $15 \mathrm{MeV}$ alphas on ${ }^{42} \mathrm{Ca}$ calculated at the matching radius as a function of the angle of incidence. (The beam goes
in at $180^{\circ}$. 
Fig. IIC5.1 shows a calculation made for $15 \mathrm{MeV}$ alphas on ${ }^{42} \mathrm{Ca}$. The radial component $J_{\rho}$ is shown as a function of the angle $\theta$ of the incident alpha particle. Here, $J_{\rho}$ was calculated at the (matching) radius of $\mathrm{R}=11.3 \mathrm{fm}$ where the riclear potential is negligible. The negative contribution is due to the incident beam. The positive one peaks around $90^{\circ}$ and indicates the reflected flux. The integral of the curve in Fig. IIC5.1 gives us, according to Eq. (2), the absorbed flux.

Table IIC5.2. Parameters extracted in the analysis of the $\alpha+{ }^{42} \mathrm{Ca}$ system

\begin{tabular}{cccccc}
\hline$E_{C M}(M e V)$ & $R_{0}(\mathrm{fm})$ & $r_{0}(\mathrm{fm})$ & $\sigma_{O M}(\mathrm{mb})$ & $\sigma_{D R A F}(\mathrm{mb})$ & $\Delta(\%)$ \\
\hline 8.0 & 8.0 & 2.30 & 402.62 & 345.24 & -14.26 \\
10.0 & 7.9 & 2.27 & 702.16 & 614.70 & -12.46 \\
12.0 & 7.7 & 2.22 & 900.65 & 784.47 & -12.90 \\
15.0 & 7.5 & 2.16 & 1085.91 & 933.45 & -14.04 \\
20.0 & 7.2 & 2.07 & 1248.54 & 1034.86 & -17.11 \\
\hline
\end{tabular}

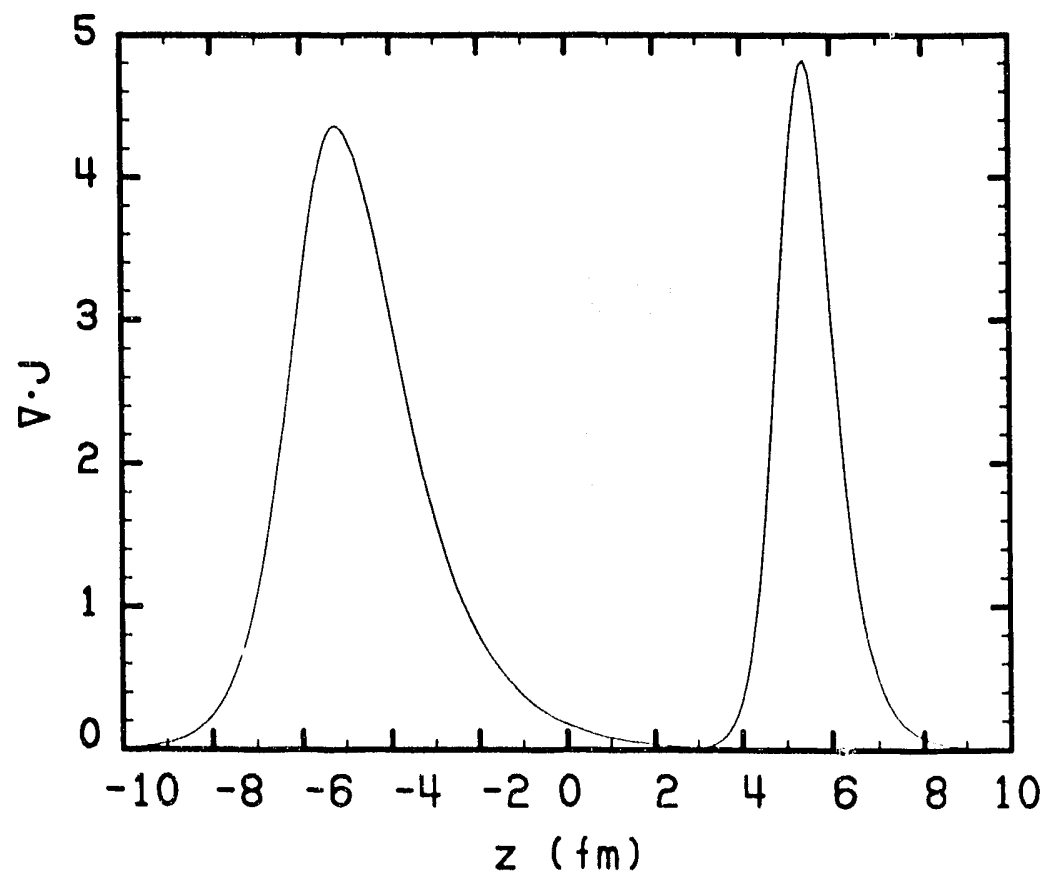

Figure IIC5.2. Divergence of the probability current (apart from a factor of $2 / \hbar$ ) along the beam direction for $42 \mathrm{MeV}$ alphas on ${ }^{42} \mathrm{Ca}$. The beam is incident from the left. 
Examination of the positive and negative contributions of the integral of Eq. (2) shows that they both increase monotonically as a function of the distance R. However, their sum tends asymptotically to the optical model absorption cross section. This behaviour makes difficult the determination of the absolute magnitude of the the reflected flux. The ratio of the negative to positive contributions decreases as a function of the distance. This way, we determine a distance $R_{0}$ at which reflections start. However, it would be unfair to assume that all of the flux for $\mathrm{r} \geq R_{0}$ leads to reflections because some absorption of the reflective components may still occur. This has been inferred from the examination of divergence plots [2] similar to the one shown in Fig. IIC5.2.

Making the assumption that all flux for $\mathrm{r} \geq R_{0}$ leads to reflections, we obtain an upper limit of the reflections in the optical model at a given energy. We then calculate the "true" optical model absorption cross section using the DRAF method [3] with $R_{0}$ as the fusion radius. The radius $R_{0}$ can be parametrized as $R_{0}=r_{0} A^{1 / 3}$. In our example of ${ }^{42} \mathrm{Ca}$, the calculated $\sigma(O M), \sigma(D R A F)$, and radius parameters $r_{0}$ are tabulated in Table IIC5.2 at selected energies. As expected, the radii at which reflections start decrease with increasing particle energy. This results in a $\sigma(D R A F)$ value which is closer to $\sigma(O M)$ at lower energies.

$$
\vec{j} \text { for } 42 \mathrm{MeV} \alpha+{ }^{42} \mathrm{Ca}
$$

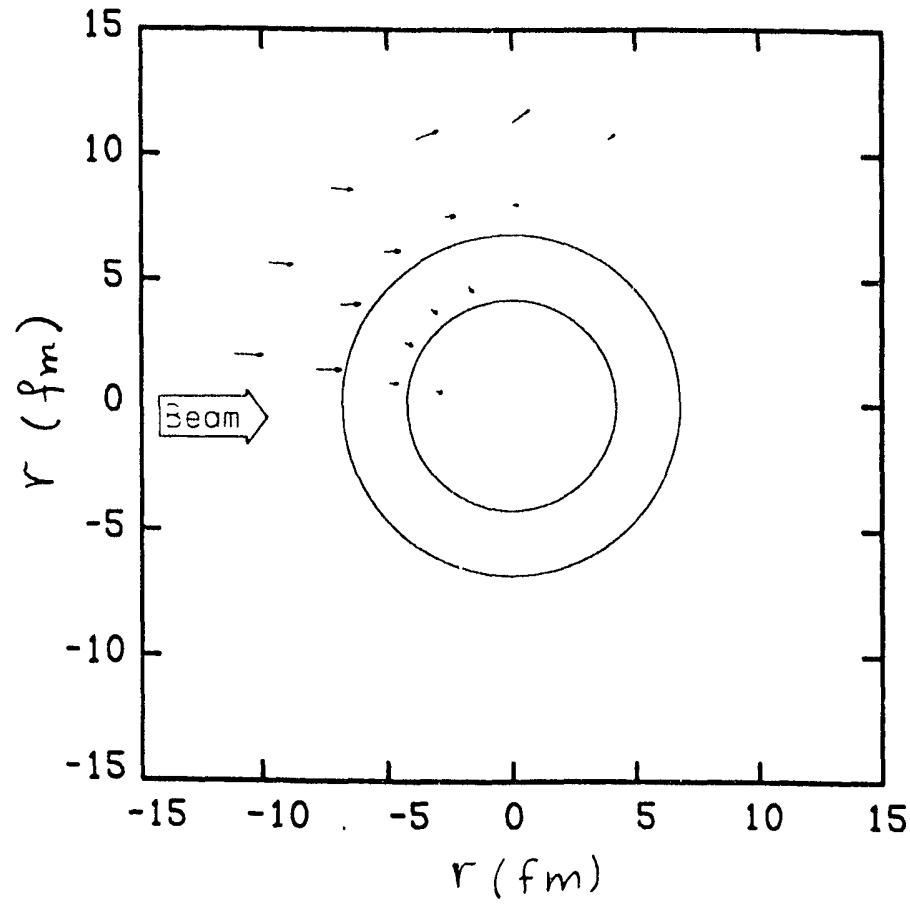

Figure IIC5.3. Plot of the probability current density vector $J$ for $42 \mathrm{MeV}$ alphas on ${ }^{42} \mathrm{Ca}$. The circles correspond to the $10 \%$ and $90 \%$ of the central value of the imaginary potential. The outermost arrows were drawn at a distance equal to the matching radius where reflections are apparent. 
A calculation of the azimuthal component $J_{\theta}$ gives the representation of the probability current density shown in Fig. IIC5.3. The figure is symmetric with respect to the horizontal axis. The circles in the the figure show the 10 and $90 \%$ of the central value of the imaginary potential. The outermost arrows were drawn at a distance equal to the matching radius where reflections are strong. The magnitude of $J$ gets smaller as the distance to the center decreases. This indicates absorption.

Summarizing, we have been able to study the percentage of transparency contributions in the optical model absorption cross section in the case of alpha particles. The transparency was found to increase with bombarding energy. The method will be applied next to the case of ${ }^{117} \mathrm{Te}$.

This work was done exclusively by N. Nicolis (this grant), with useful discussions with the nuclear chemistry group.

[1] J.M. Alexander, M.T. Magda and S. Landowne, Phys. Rev. C42, 1092(1991).

[2] I.E. McCarthy, Nucl. Phys. 10,583(1959).

[3] T. Udagawa, B.T. Kim and T. Tamura, Phys. Rev. C32, 124(1985).

[4] D.F. Jackson and C.G. Morgan, Phys. Rev. 175, 1402(1968). 


\title{
IID. REACTION MECHANISM STUDIES
}

\section{IID1. The Mechanism for the Disassembly of Excited ${ }^{16} \mathrm{O}$ Projectiles into} Four Alpha Particles - R.J. Charity, J. Barreto, L.G. Sobotka, D.C. Sarantites, D.W. Stracener, A. Chbihi, and N.C. Nicolis [WU], R. Auble, C. Baktash, J.R. Beene, F. Bertrand, M. Halbert, D.C. Hensley, D. Horen, C. Ludermann, M. Thoennessen and R. Varner [ORNL]

The decay of excited nuclei into many fragments is a topic of considerable interest at present. The models for explaining this type of decay include both prompt breakup and sequential emission of the fragments. It is important to find clear signatures for the various modes of disassembly. Such a technique is to study the relative velocities between the fragments. If the fragments are initially in close proximity their subsequent interaction is dominated by the mutual Coulomb repulsion, which leads to a suppression of small relative velocities (relative ernission angles). In contrast, for a seculuential decay, the time intervals between emissions will be long and the fragments do not experience significant mutual interactions. Therefore the small relative velocities are not suppressed. Harmon et. al [1] have measured relative angles of $\alpha$-particles produced in the disassembly of ${ }^{16} \mathrm{O}$ projectiles, and concluded that a sequential decay mechanism arcounts for this decay.

In this work :ve also studied the breakup of ${ }^{16} \mathrm{O}$ into $4 \alpha$ 's, but both our experimental approach and analysıs had some new important features, which are summarized in the following abstract of a paper (see Appendix M) accepted for publication in the Physical Review C.

\begin{abstract}
The decay of excited ${ }^{16} \mathrm{O}$ projectiles into the four alpha particle exit channel has been investigated. The projectiles, with bombarding energies of $\mathrm{E} / \mathrm{A}=25 \mathrm{MeV}$, were excited through peripheral interactions with ${ }^{159} \mathrm{~Tb}$ target nuclei. A $4 \pi$ counter was used to detect both the projectile alpha particles and the light charged particles evaporatel from the target nucleus. Criteria for selecting true ${ }^{16} \mathrm{O} \rightarrow 4 \alpha$ events with the minimum contamination from incorrectly identified events were examined. The distribution of relative angles between the four alpha particles in their center of mass frame was compared to simulations of ${ }^{16} \mathrm{O}$ decay by sequential decay mechanisms. The relative angles were shown to be consistent with a sequential evaporation mechanism if a nonzero angular momentum is assumed for the excited projectile. The momentum distribution of the alpha particles in the reaction plane was examined and found to be anisotropic for high projectile excitation energies. This anisotropy was shown to be consistent with Coulomb interactions between the first emitted alpha particle and the target nucleus, which would indicate that the lifetime of the projectile is very short; on the order of $10^{-22} \mathrm{~s}$.
\end{abstract}

Our group provided the experimental data, which were acquired by $\mathrm{D}$ Sarantites and D. Stracener in a summer visit at ORNL. The data analysis was done by J. Barreto and the simulations and interpretation by $\mathrm{R}$. Charity.

[1] Harmon et. al., Phys. Lett. B235, 234 (1990). 
IID2. Binary Character of Highly Dissipative ${ }^{209} \mathrm{Bi}+{ }^{136} \mathrm{Xe}$ Collisions at E/A=28.2 MeV - B. Lott, S.P. Baldwin, B.M. Szabo, B.M. Quednau, W.U. Schröder, J. Töke [ROCH], L.G. Sobotka, J. Barreto, R. Charity, L. Gallamore, D.G. Sarantites, D.W. Stracener [WU], and R.T. de Souza [IU].

An interesting question in recent years has been whether novel reaction mechanisms exist in intermediate energy heavy-ion collisions. Despite the many scenarios possible, the answer to this question is still unknown. In view of the fact that a considerable fraction of the reaction cross section bears the characteristics of partially damped inelastic reactions, it is clear that powerful reaction filters will be needed to resolve these issues.

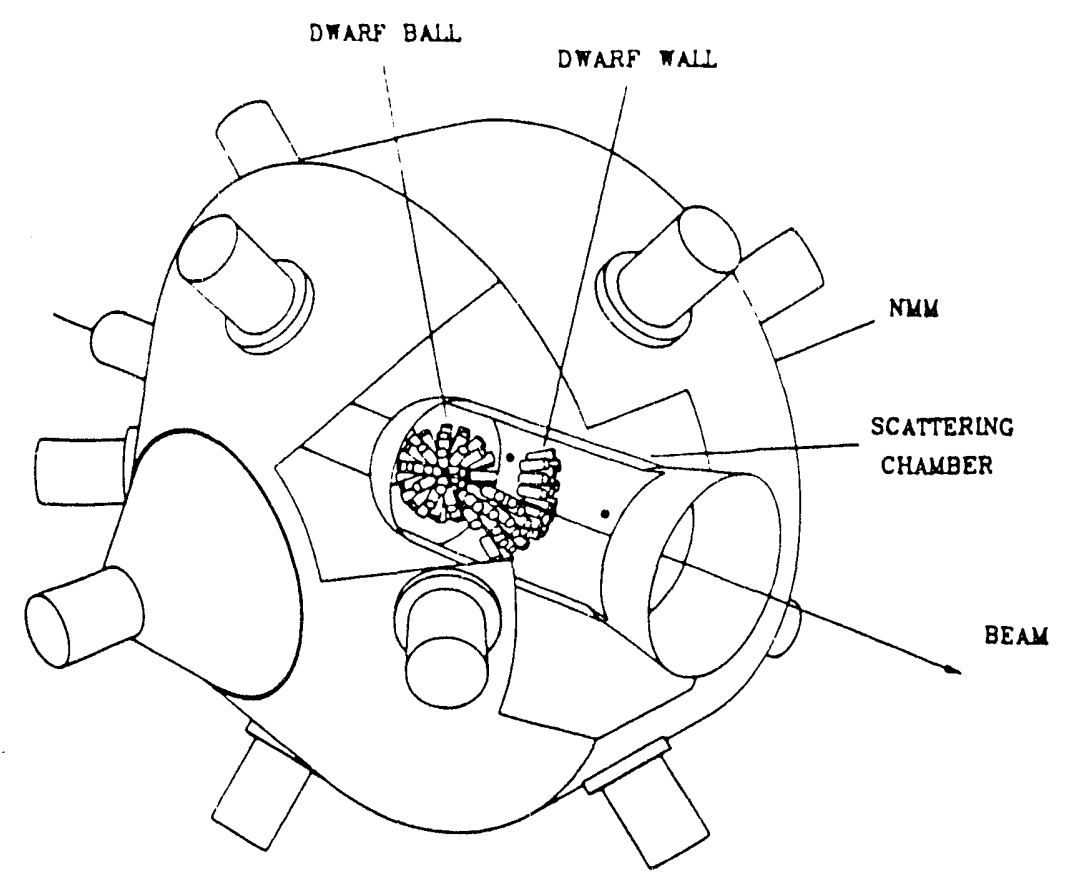

Figure IID2.1. The experimental setup.

For this purpose we combined simultaneously exclusive neutron and charged particle $4 \pi$ measurements and studied the reaction ${ }^{209} \mathrm{Bi}+{ }^{136} \mathrm{Xe}$ at $28.2 \mathrm{MeV} /$ nucleon. The apparatus is shown in Fig. IID2.1. The neutrons were recorded by the $900 \mathrm{l}$ Gd-loaded liquid scintillator tank (University of Rochester), while the charged particles were detected by the WU Dwarf system.

The experimental joint multiplicity distribution between charged particles and neutrons is shown in Fig. IID2.2. As would be expected for such a heavy system, for peripheral collisions predominantly neutrons are emitted. For more dissipative collisions charged particle ernission becomes important. The stars in Fig.IID2.2 are the result of GEMINI calculations for different total energy losses. The calculations were not corrected for the efficiency of the detector system. The last star corresponds to a total of $1.2 \mathrm{GeV}$. This energy is much less than the total kinetic energy above the entrance channel Coulomb 


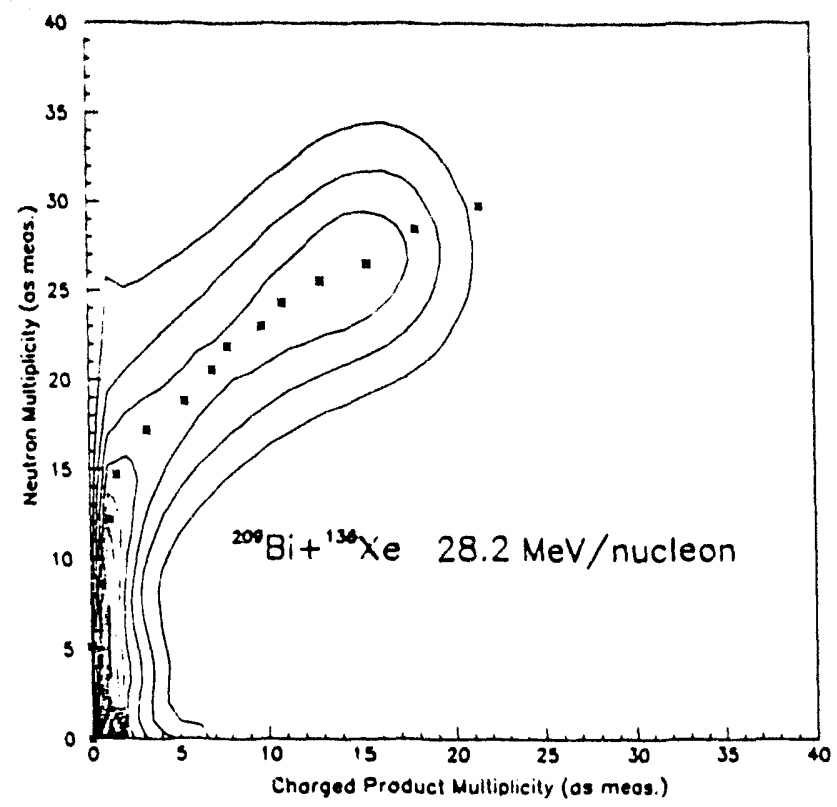

Figure IID2.2. Experimental joint multiplicity distribution (uncorrected for instrumental response) of neutrons and charged particles.

barrier $(2.3 \mathrm{GeV})$. This would seem to suggest that damping is far from complete. Some support for this conclusion can be found in the light particle invariant cross section plots which show blurred images of only moderately damped projectile and target sources (See Fig. 2 in Appendix N). However, there are two reasons to doubt this conclusion. Firstly, we believe that use of GEMINI at this excitation energy region is inappropriate. Secondly, other studies which we have done (Sobotka's analysis of our GANIL data for the ${ }^{8.4} \mathrm{Kr}$ induced reactions at $45 \mathrm{MeV} / \mathrm{A}$ ) indicate that the larger the charge of the detected species, the greater the apparent damping. Therefore, the extent of damping is still an open question. However, it is clear that binary or entrance channel dominated dynamics persist in the energy region of this work.

B. Lott and S. Baldwin (from Rochester) have done the bulk of the analysis for this project. A significant portion of their work was done during a one month visit to Washington University.

This work is contained in Phys. Rev. Letter [1] (see Appendix N).

1. B. Lott, S.P. Baldwin, B.M. Szabo, B.M. Quednau, W.U. Schröder, J. Tóke, L.G. Sobotka, J. Barreto, R. Charity, L. Galiamore, D.G. Sarantites, D.W. Stracener, and R.'T' de Souza, Phys. Rev. Lett. 68, $3141(1992)$. 


\section{IID3. Particle Multiplicity Dependence of the High Energy Photon Production} in a Heavy-Ion Reaction - L.G. Sobotka, L. Gallamore, A. Chbihi, D.G. Sarantites, D.W. Stracener [WU], W. Baner, D.R. Bowman, N. Carlin, R.T. d Souza, C.K. Gelbke, W.G. Gong, S. Hannuschke, Y.,D. Kim,W.G. Lynch, R. Ronnigen, M.B. Tsang, F. Zhu [MSU], J.R. Beene, M.L. Halbert and M. Thoennessen [ORNL].

This study of bremstrahlung production as a function of charged-particle multiplicity was done at MSU using the Dwarf System and the ORNL BaF $2 \gamma$-ray detection array. The experiment was analyzed by L.G. Sobotka and L. Gallamore and a paper was submitted for publication. The abstract is given below and a preprint is attached as Appendix P.

\footnotetext{
Abstract: The production of high energy photons in an intermediate energy heavy ion reaction (65 $\mathrm{MeV} /$ nucleon ${ }^{40} \mathrm{Ar}={ }^{93} \mathrm{Nb}$ ) is studied by characterizing the events, which produce the photons, by the forward and backward, light and heavy, charged particle production. While the absolute yield of high energy photons increases with increasing charged-particle multiplicity, the spectral shape is found to be almost independent of multiplicity. This indicates that the fundamental photon production mechanism is insensitive to the impact parameter but that the production process is more probable for the more violent central collisions. These data are compared to predictions of reaction simulations based on the Boltzmann-Uehling-Uhenbeck equation. These calculations reproduce the overall yield of photons over $30 \mathrm{MeV}$ but underpredict the inverse slope parameter. This comparison also suggests strong particle multiplicity fluctuations at fixed impact parameter.
} 


\section{INSTRUMENTATION AND ANALYSIS}

\section{IIIA. ADVANCES IN INSTRUMENTATION}

\section{IIIA1. "Microball" a $4 \pi$ Channel Selection Device: An Extension of the Dwarf Ball and Wall System - L.G. Sarantites, P.F. Hua, A. Kirov, D.W. Stracener, J. Elson, and L.G. Sobotka [WU].}

From experimental work presented in this report it has become clear that the Dwarf Ball and Wall (DBW) system has been well suited for low to intermediate energy reaction studies, and as a tool for spectroscopic studies via its channel selection capability. Furthermore, in the overlap area between these two fields, the so called "interface between structure and reactions", the DBW system is capable of making the most significant contributions. For these reasons we were very much interested in improving the performance and counting rate capabilities of the DBW system most appropriate for these low energy studies. We have replaced 18 of the 4- and 8-mm CsI(T $)$ DBW detectors (those in front of Ge detectors in the Spin Spectrometer) with $2 \mathrm{~mm}$ thick ones. This reduces absorption of the low energy $\gamma$ rays that are recorded in the Ge detectors.

Design studies for a new device termed "Microball" were carried out this year. Based on our considerable experience with the DBW system for spectroscopic studies, it became clear that a new device was called for. This device is aimed in vastly improving several of the drawbacks of the DBW system.

There are several areas that require major improvement over the DBW system:

1. Absorption and scattering: We need to minimize the mass of the detectors and associated components surrounding the target. This will improve efficiency for discrete $\gamma$-rays through the device. More importantly, it will reduce scattering (see discussion below).

2. Stability: The gain stability of the device must be better than what can be achieved with photomultiplier tubes and passive voltage dividers presently in the DBW system.

3. Counting Rates: We must increase the overall counting rate capabilities of the device over that of the DBW system. This can be done by equalizing the counting rates for each particle det ctor so that the device can keep up with the rates acceptable for Ge detectors. This improves the overall counting rate capabilities of the system considerably, and allows the device to be used in conjunction with low efficiency external triggering devices.

4. Complete $4 \pi$ coverage: This is necessary for weak channel selection when multiple charged particle emission is involved, as for example the (HI, $4 \mathrm{p}$ ) reaction.

Absorption and scattering are key factors in using a particle detecting device for spectroscopic studies. Below we summarize some measurements that clearly demonstrate the seriousness of the scattering on the performance of Compton suppressed Ge detectors. We mentioned above that we reduced absorption for the DBW system by replacing detectors with thinner ones. In addition, it was thought that this would minimize the forward 
Compton scattering, which removes counts from the full energy peaks and increases the undesirable Compton background. To investigate this quantitatively, we have measured the peak-to-total ratio for the Compton suppressed Ge detectors at HHIRF in the Spin Spectrometer, with and without the Dwarf Ball in place.

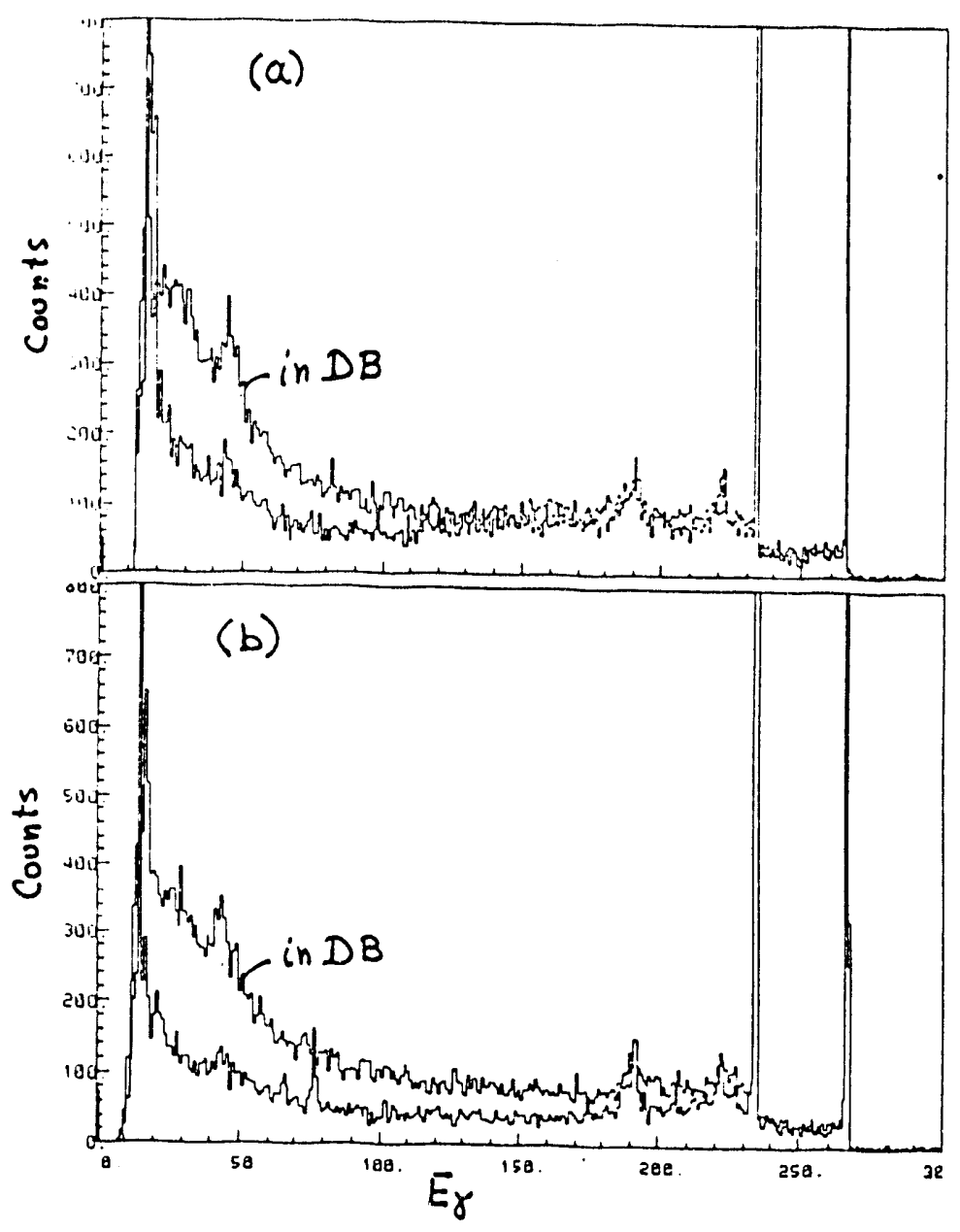

Figure IIIA1.1. Compton suppressed Ge spectra taken with a ${ }^{60} \mathrm{Co}$ source inside the Dwarf Ball (top spectra) and with the source outside the Dwarf Ball (bottom spectra) for two different Ge detectors (a) and (b) with $\mathrm{BGO}$ antiCompton shields. The compared spectra are normalized to the same peak area in each case. The peak maxima are off scale. The observed different behavior between the two detectors is not understood.

In Fig. IIIA.1 we show Compton distributions from two Ge detectors suppressed with $\mathrm{BGO}$ shields. The top spectrum in each case is taken with a ${ }^{60} \mathrm{Co}$ source inside the Dwarf Ball and the bottom one with the source outside. The spectra are normalized to the same full energy peak area. It is seen that for the detector in (b) the increased Compton background goes up to $\approx 1.1 \mathrm{MeV}$ whereas in (a) the increase is limited to energies below $\approx 500 \mathrm{keV}$. We do not understand the different behavior of these two detectors, but out of 
18 detectors tested 6 showed the pattern in (a) and 12 that of (b). We then took out the CsI detector in front of a Ge detector and found that the increase in background was $\approx$ $2 / 3$ of what it was with the $2 \mathrm{~mm}$ CsI detector in place. This clearly indicates that large angle scattering is responsible for the increase in the Compton background. To test this idea we shielded the antiCompton shield with a $6.0 \mathrm{~mm}$ and $2.5 \mathrm{~cm}$ thick $\mathrm{Pb}$ shields (not in very good geometry). The results are shown in Fig. IIIA1.2. It is clear that substantial reduction of the extra background can be obtained by shielding the BGO suppressors. The improvement would be better if the Ge detectors could be collimated. Some of this can be accomplished by better collimation than that used in these measurements.

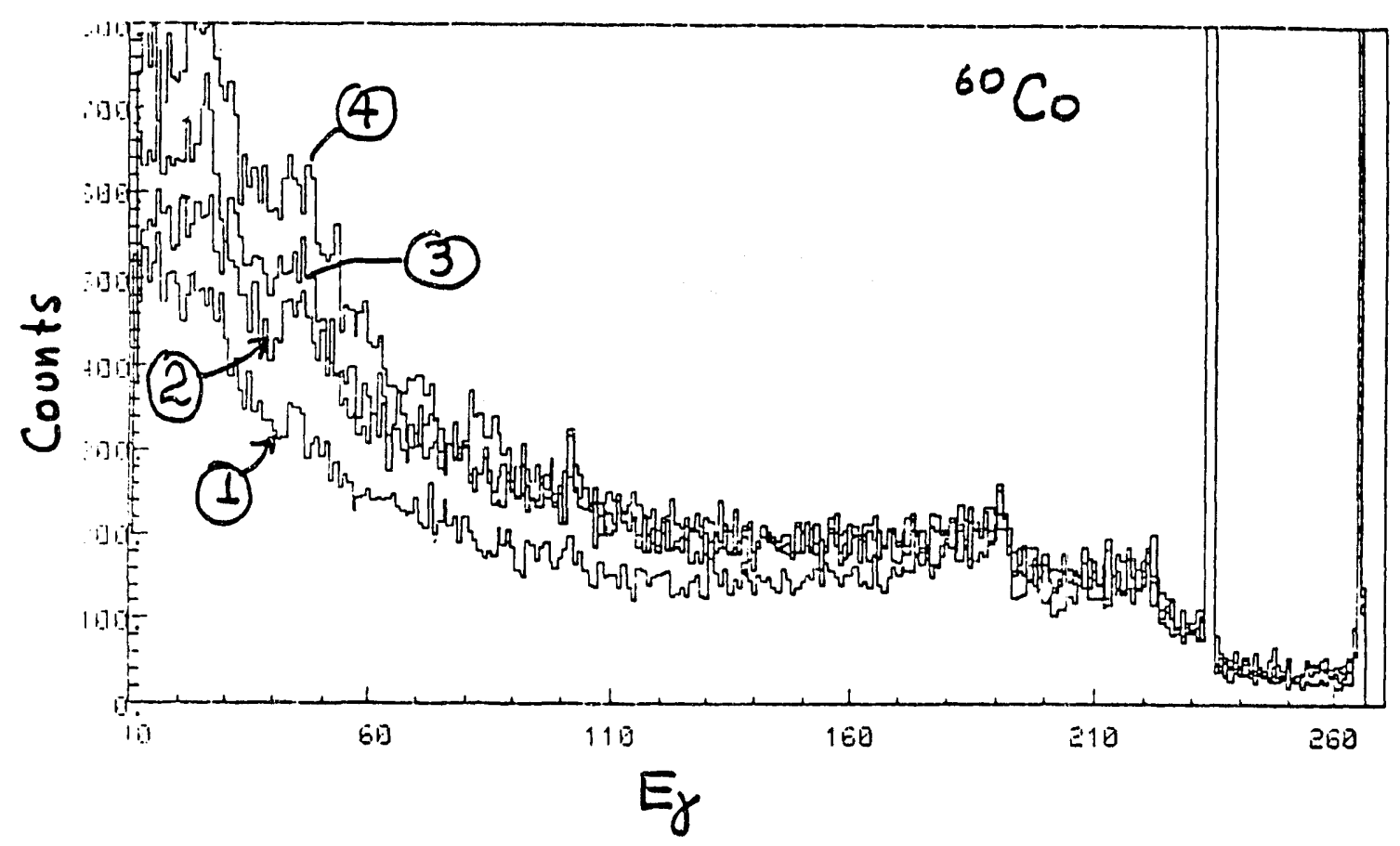

Figure IIIA1.2 Normalized Compton suppressed spectra to the same peak area. $1=$ source outside the Dwarf Ball, 2 = source inside the DB with $2.5 \mathrm{~cm} \mathrm{~Pb}$ shield, $3=$ source inside the DB with 6.0 $\mathrm{mm} \mathrm{Pb}$ shield, and $4=$ source inside the $\mathrm{DB}$ but with no shielding.

From these results it is clear that a substantial reduction of the total DB mass is required. This can be done by (a) replacing all the CsI detectors (4 and $8 \mathrm{~mm}$ ) with 2 $\mathrm{mm}$ CsI ones and (b) replacing the photomultiplier tubes with PIN diode readout. The prototype detectors that we tested allow a reduction of the Dwarf Ball mass by a factor of 8 (the CsI is 2 to 3 times thinner and all support material is plastic).

In order to test these concepts we have made measurements with prototype detectors and PIN diodes for readout in a number of geometries. We have used the Hamamatsu S3590-3 PIN diode $(10 \times 10 \mathrm{~mm}$ sensitive area, $300 \mu \mathrm{m}$ thick $)$. Two $\mathrm{CsI}(\mathrm{T} \ell)$ geometries 
were used, one was $1 \mathrm{~cm}^{2}$ and the second had trapezoidal shape $(2.1+1.4 \mathrm{~cm}$ bases, 1.7 $\mathrm{cm}$ wide). Two different preamplifiers were tested, one from Hamamatsu and the second from an MSU CsI hodoscope. We found energy resolution comparable to that in the Dwarf Ball system (7.5\% local FWHM/pulse-height for $8.9 \mathrm{MeV} \alpha$ particles) when the square scintillator was used either directly on the PIN diode or via a matched square light guide $6 \mathrm{~mm}$ thick. Similar results were obtained when the trapezoidal scintillator was used with a matched in shape $6 \mathrm{~mm}$ thick light guide. In contrast, when the trapezoidal scintillator was coupled directly on the $1 \mathrm{~cm}^{2}$ diode the energy resolution was very poor.

Further tests were made for the effect of cable length between the PIN diode and the preamplifier. We found the same unaltered energy resolution for no cable, or 30 and 100 $\mathrm{cm}$ long cables. This is encouraging because one does not need to have the preamplifier directly coupled to the PIN diode, thus further minimizing the mass around the target.

We also tested the temperature dependence of the energy resolution, by cooling both the PIN diode and the preamplifier. No significant difference was found for operation between 0 and $40{ }^{\circ} \mathrm{C}$.

Returning to the gain stability issue, we note that the PIN diode readout provides the necessary stability over that from a photomultiplier with a passive voltage divider.

The issue of the counting rate improvement is substantially more complex. Upto now we have used the CsI $(\mathrm{T} \ell)$ scintillators in the DBW system because of the excellent particle identification, especially for very low energies. This is offset by the limited counting rate performance $(<10,000 \mathrm{c} / \mathrm{s}$ per element at best). The rate problem in the DBW arises from the equal solid angle subtended by each detector, which due to the forward focusing causes the forward detectors to be the limiting ones. The obvious solution might be choosing a faster scintillator, but in view of the desire to have good identification, low thresholds and inexpensive close packing into $4 \pi$, we must exclude most of the faster scintillators and the combined phoswitch $(\triangle \mathrm{E} \times \mathrm{E})$ concept.

A viable solution that remains is to use a sufficiently high segmentation that increases with decreasing laboratory angle so that for a typical fusion reaction and projectile energy the counting rate in each detector is approximately equal. With these constraints we find that a device with 9 rings of detectors ( 96 detectors in total) meets all these requirements. A simple calculation shows that if $10,000 \mathrm{c} / \mathrm{s}$ is taken as the limiting Ge rate, then assuming that $\left\langle M_{\gamma}\right\rangle=20$ we find that $1.1 \times 10^{5}$ events/sec will be a typical reaction rate (this result is obtained for $110 \mathrm{Ge}$ Gammasphere detectors covering $50 \%$ of $4 \pi$, or for any other current array with fewer comparable detectors). Assuming that the average particle multiplicity is 3 (a high estimate) and a segmentation of 96 detectors gives a rate of $\sim 3,000 \mathrm{c} / \mathrm{s}$ per $\mathrm{CsI}(\mathrm{T} \ell)$ element, which is a comfortable rate for this scintillator. A working drawing for the design of such a devise is shown in Fig. IIIA 1.3. The CsI(T $\ell$ ) scintillators are $2 \mathrm{~mm}$, 


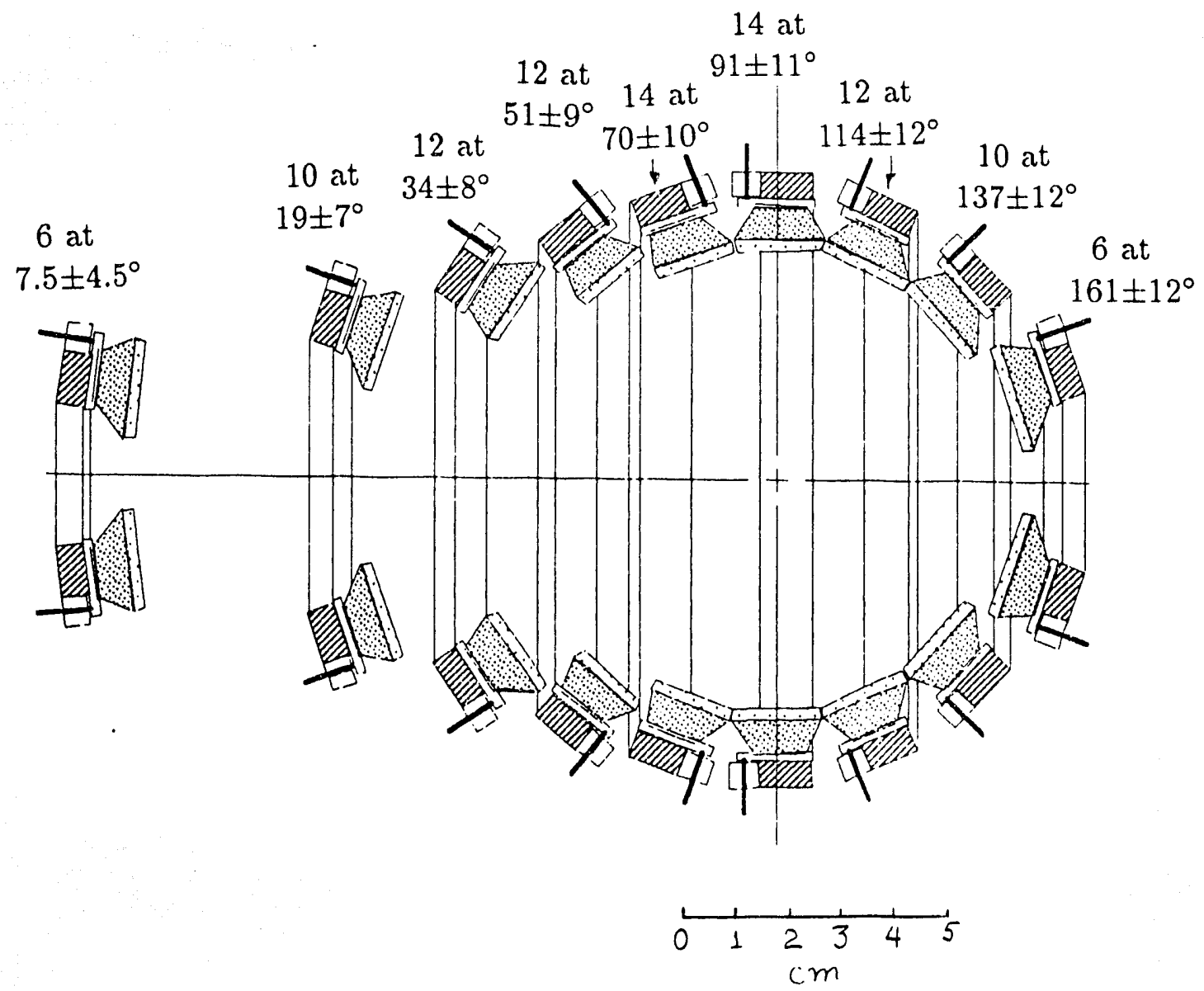

Figure IIIA1.3. Design of the Microball arrangement. The number of CsI(T $\ell$ ) detectors in each of the 9 rings and the central and half angles are given. The total number of detectors is 96 (six banks of 16 of electronics are needed to run the device).

the thickness of the lucite light guides is $5 \mathrm{~mm}$, and the photodiode support rings are 4 mm thick, with many hollow sections.

Another aspect of the counting rate improvement is based on new features in our DBW electronics. Up to very recently we have been using the SLOW (integration of the current pulse from 0-400 ns) and the LONG (or TAIL, 1500-3000 ns) parameters for light chargedparticle identification. While this gives good separation, it suffers from the LONG ( $7 \mu$ s fall time) long time component, which at high rates gives a baseline shift. We have developed an alternate method for pulse-shape discrimination, which provides (a) adequate particle identification at low energies (b) permits a significant increase of the counting rate, and (c) offers at no extra cost pile-up rejection.

The earlier version of our discriminators provide individual ECL outputs with widths equal to the time over the discriminator threshold. These discriminators have a built in shaper with a selectable time constant. If this time is set to $\approx 2 \mu \mathrm{s}$, then the tails of the pulses from different types of particles will cross the discriminator level at different times 
because of the variation in the light from the two components of the $\mathrm{CsI}(\mathrm{T} \ell)$. This provides the desired pulse shape capability with a $\approx 2 \mu$ s shaping constant and allows performance at higher rates. We hope to increase the rates from $3-4 \mathrm{Kc} / \mathrm{s}$ to $15-20 \mathrm{Kc} / \mathrm{s}$. This functionality is accomplished with the use of the recently purchased LeCroy 2277 Multihit TDC's. These 32 channel real time TDC modules can register 16 hits per channel, which may be either on the negative or the positive going edges of the discriminator output signal. The time over threshold is then directly determined from the time difference between the first two hits (the first going negative and the second going positive). Subsequent hits are due to recrossings of the threshold levels. These recrossings are due to either statistical light fluctuations in the original signal (with short duration) or due to pile-up of another pulse (long time difference). This pulse shape discrimination resolution by this technique is not as good as the standard method, but when high rates and pileup rejection are needed it is adequate for low energies where only $\alpha$ particles and protons are emitted.

Another improvement in our DBW electronics and in the Mini Wall electronics is the use of a new set of 2-dimensional discriminators. A set of 8 16-channel modules were constructed from funds from this grant. They provide several new capabilities (a) computer controlled setting of thresholds (b) rejection of pulses narrower than $\approx 9$ nsec (used for rejection of pulses due to electrons) (c) an adjustable time interval in which it does not refire, (d) 16-channel ECL outputs, and (e) an OR from any of the 16 channels (NIM output). The rejection of the electron pulses was found to be very valuable, because it allows us to lower the energy thresholds considerably. These modules, however, do not provide the time over threshold. We plan to modify the earlier version of our discriminators to incorporate the rejection of narrow pulses.

\section{IIIA2. A Novel X-ray Detector- L.G. Sobotka, and D.G. Sarantites [WU].}

In Sect. IIB5 we discussed the use of an atomic X-ray clock for measuring the lifetimes of subbarrier $\alpha$-particle emission. The small probability of creating the $\mathrm{K}$ vacancy during the incoming atomic collision is only of the order of $1 \%$, (see the section on this subject in the proposal.) This makes all such measurements difficult. In addition considerable background in the X-ray region of interest (40-100 keV) is created from the fusion or fission $\gamma$ rays and their Compton scattering distributions. Further complication arises from $\mathrm{X}$-rays following internal conversion. The latter can usually be eliminated by requiring coincidence with an $\alpha$ particle or fission fragment, depending on the particular interest at hand.

Low energy $\gamma$ rays with multipolarities other than E1 in the X-ray energy region will be slow enough to shadow block (see proposal). If shadow blocking is employed, the major background will be the Compton photons. Therefore effective Compton suppression is extremely important. Due to experimental difficulties, our previous experiments did not make use of Compton suppression on the X-ray detectors. In our future experiments we will not only use Compton suppression, but also use an X-ray detector which significantly improves the suppression as compared to standard designs. 


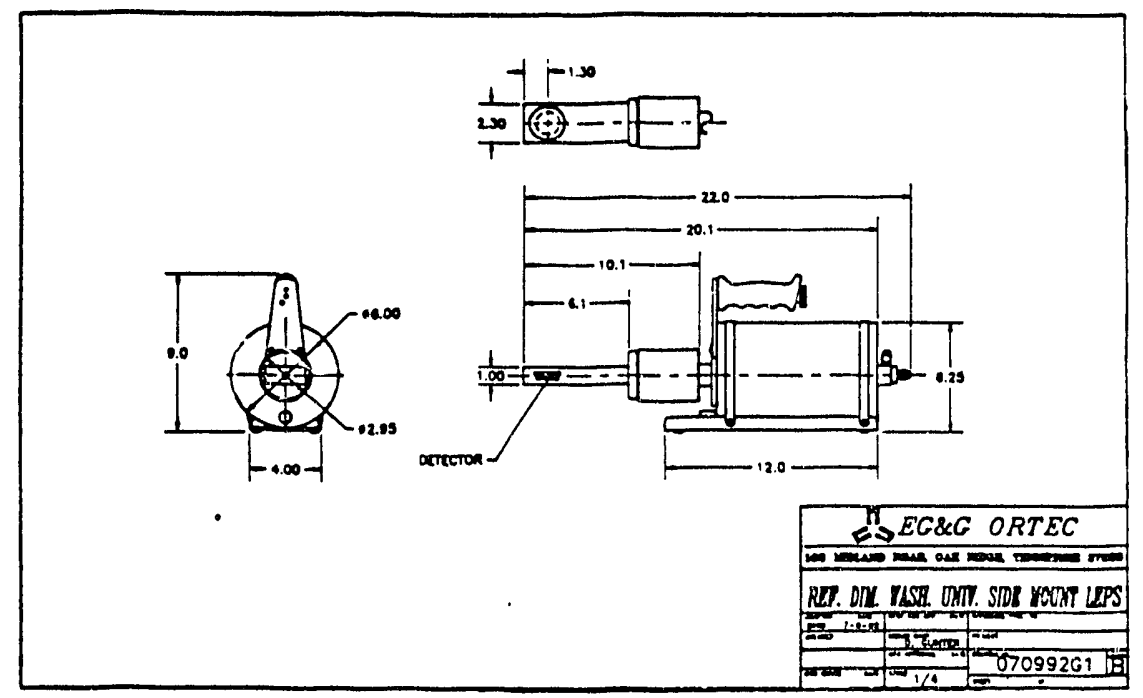

Figure IIIA2.1. Design of the transversely mounted planar Ge X-ray detector.
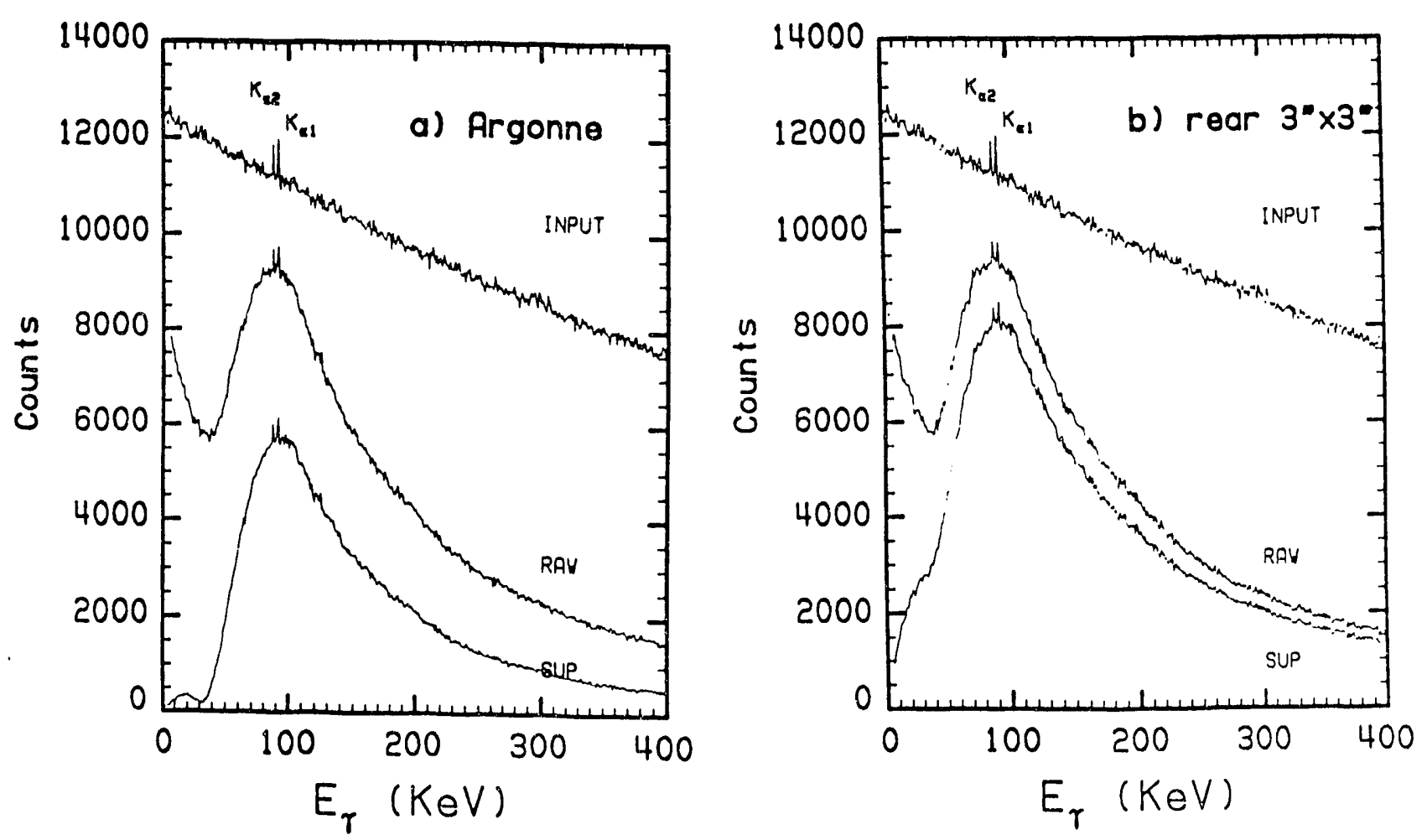

Figure IIIA2.2. GEANT simulations of the transversely mounted planar Ge x-ray detector with both a) an Argonne type suppressor and b) a simple 3 " $x 3$ " NaI(T $\ell$ ) veto detector behind the active region. These simulations each have 2 parts of $\mathrm{K} X$-rays to 10,000 parts of a fission-like $\gamma$ spectrum. $\mathrm{A} \mathrm{Cu}$ absorber has been included to reduce the counting rate from target $\mathrm{X}$-rays, which have not been included in the simulation.

In the standard design of planar Ge $\mathrm{X}$-ray detectors the crystal axis is radial and 
collinear with the cryostat. This geometry prevents the placement of any scintillation veto element directly behind the Ge crystal. Needless to say, this is the most important region to have suppression detectors. No planar Ge detector design on the market allowed for the placement of a suppression element in the $0^{\circ}$ region, so we designed one.

Figure IIIA2.1 shows our design. The axis of the crystal is perpendicular to the cryostat. The can has a rectangular cross section of 1 " by 2 ", with the axis of the crystal along the 1 " dimension.

This design was simulated with GEANT [1] before placing the order for this detector. Some of the simulations are shown in Fig. IIIA2.2. The initial photon distribution was chosen to resemble that from fission (but without any structure) with the addition of 2 parts per $10,000 \mathrm{~K}$ x-rays from the parent nucleus. This input spectrum is shown as the top curve in both parts a) and b). The "raw" spectrum from this detector, with a $1 \mathrm{~mm}$ $\mathrm{Cu}$ absorber placed in front of the active region, is also shown in both parts of the figure as the middle curve. The bottom curves are the Compton suppressed spectra from a) an Argonne type - full suppressor and b) a simple 3"x3" $\mathrm{NaI}(\mathrm{Tl})$ placed behind the Ǵe. The value of the full suppressor is clearly seen. In addition, the value of suppressing directly behind the active element is also seen, in that even a 3" 33 " $\mathrm{NaI}(\mathrm{T} \ell)$ crystal provides some suppression. (The funds for this order were split between Sobotka's PYI award and this grant.)

A $800 \mathrm{~mm}^{2}$ detector of this design has now been delivered from ORTEC. We have tested the detector and achieved resolution better than the manufacturer's specifications. Unfortunately, the detector was not delivered in time to run our approved experiment at HHIRF before this facility shut down. We will submit this proposal to Argonne, (see proposal.)

[1] R. Brun et al. GEANT3 Users Guide (Data Handling Division EE/84-1 CERN,1986).

IIIA3. A Simple Channel Selecting Detector-P. F. Hua, J. Barreto, D.G. Sarantites, L.G. Sobotka [WU].

The interface between low energy spectroscopy and reactions is one which presents many interesting questions. The general issue of entrance channel effects and the population of interesting nuclear structures in deexcitation chains involving particle emission are two clear examples. Such issues are also ones which will come up time and time again with work with RIBS.

In many experiments the Dwarf Ball has been used, along with high resolution Ge detectors, as a simple channel selector as well as for a tool to investigate the interplay between charged particle energy and band population. For the latter type of study a device with good energy resolution is essential. For the former, energy resolution is not needed. Futhermore, for xn channel selection, charged-particle identification may not be as important if charged-particle tagging can be achieved. For example, often one may be 


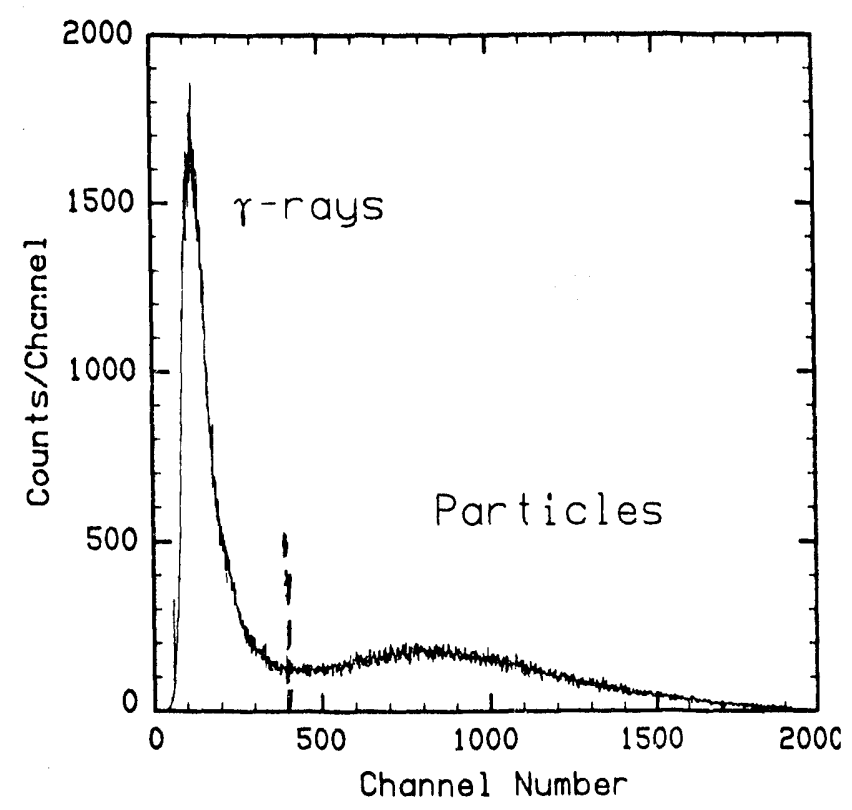

Figure IIIA3.2. Pulse-height spectrum of the detector from the reaction of ${ }^{32} \mathrm{~S}+{ }^{154} \mathrm{Gd}$. The lower energy peak is associated mainly with $\gamma$ rays from the reaction. The upper, broader peaks is associated with the detection of light-charged particles. The two peaks are reasonably well separated at the minimum.

near $100 \mathrm{kHz}$ and together, with the target in the middle, provide a geometric coverage $\simeq$ $93 \%$ of $4 \pi$.

This device is not capable of providing charged particle identification or particle energy. On the other hand, it can efficiently count charged particles (to some extent this includes fission fragments) at a high rate, with an absolute minimum of absorption and scattering of photons.

Initially it was hoped that this device, in addition to detection of light charged particles, it would respond to fission fragments and thus be used to veto fission which for neutron deficient compound nuclei is a major contributor to the $\gamma$ background. For this purpose each paddle was covered by aluminized mylar of minimal thickness $\left(170 \mu \mathrm{g} / \mathrm{cm}^{2}\right.$ thick $)$. A pulse height spectrum taken with a ${ }^{252} \mathrm{Cf}$ source (decaying by $97 \%$ by $\alpha$ emission and $3 \%$ by fission) showed fission fragment pulses extending beyond the $\alpha$ peak, but a considerable fraction of them had low pulse height due the large quenching of the light emission in the plastic.

This device was used in a recent spectroscopy experiment designed to study the high spin structure of ${ }^{182} \mathrm{Hg}$ (see Sect. IIA2). The reaction was $165 \mathrm{MeV}{ }^{32} \mathrm{~S}+{ }^{154} \mathrm{Gd}$ and thus the channel of primary interest was $4 \mathrm{n}$. The strong channels involved one or more charged particles ( $40 \%$ of the estimated fusion cross section) or fission $(\approx 50 \%)$. Before the actual run we had neglected the response of the paddles to $\gamma$ rays because of the small thickness and low $\mathrm{Z}$ of the scintillators. In the experiment we decided to store the pulse height of 


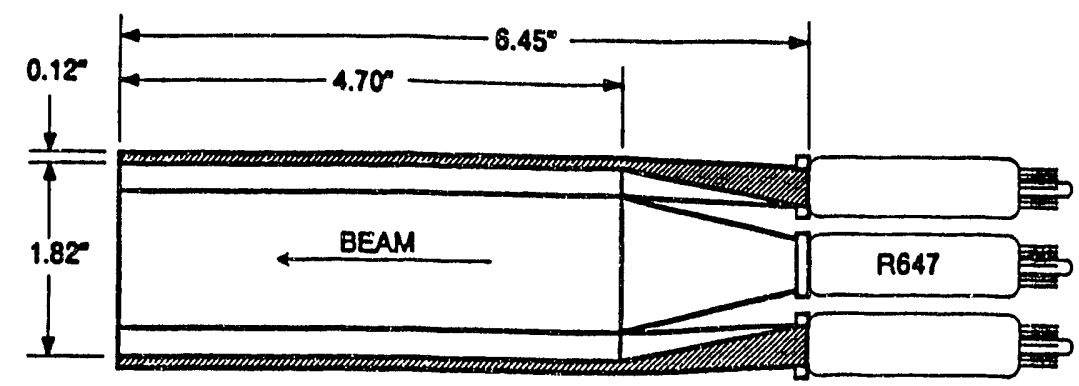

LONGTUDINAL SECTION
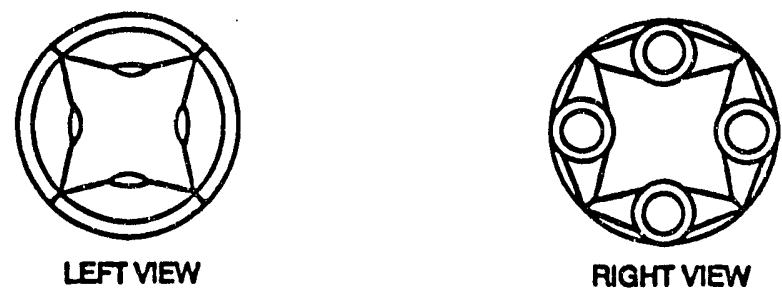

Figure IIIA3.1. Sketch of the channel selecting detector.

interested in an xn channel produced from the decay of an extremely neutron deficient compound nucleus. In this case what is required is a good charged particle veto.

In evaluating what is the best particle detector for a specific task, it is important also to keep in mind the counting rate capabilities of the various possible devices. In spectroscopy experiments, energy resolution and statistics are the two prime contenders, but Compton suppression follows very closely because it increases detection sensitivity by reducing the background. For spectroscopic studies, the advantages of particle identification and energy information provided by the $\mathrm{CsI}(\mathrm{Tl})$ of the Dwarf system are offset by (a) the substantial increase of background due to scattering of photons in the massive Dwarf Ball, and (b) by the counting rate limitations $(<10000$ counts/sec) in each element. The latter drawback can be eliminated by segmentation increasing in going to forward angles (see proposal). The scattering problem can be substantially reduced by minimizing the mass and atomic number of the charged particle detectors.

With these issues in mind we have designed, constructed, and used a simple, inexpensive, channel selecting detector, which in many ways is a beautiful complement to the Dwarf system. The device is shown in Fig. IIIA3.1. It consists of 4 optically isolated fast scintillator paddles (Bicron BC-400), which together form a cylinder. The thickness of the scintillator at the cylindrical section is only $3 \mathrm{~mm}$. The light is collected at one end of each paddle, which is shaped adiabatically (approximately constant cross section), and detected by an end-on photomultiplier tube. The abiabaticity is demonstrated by the pulse height variation of a collimated monoenergetic $\alpha$ source which was measured to be only $20 \%$ between the two ends on the scintillator paddle. Each sector can run at rates 


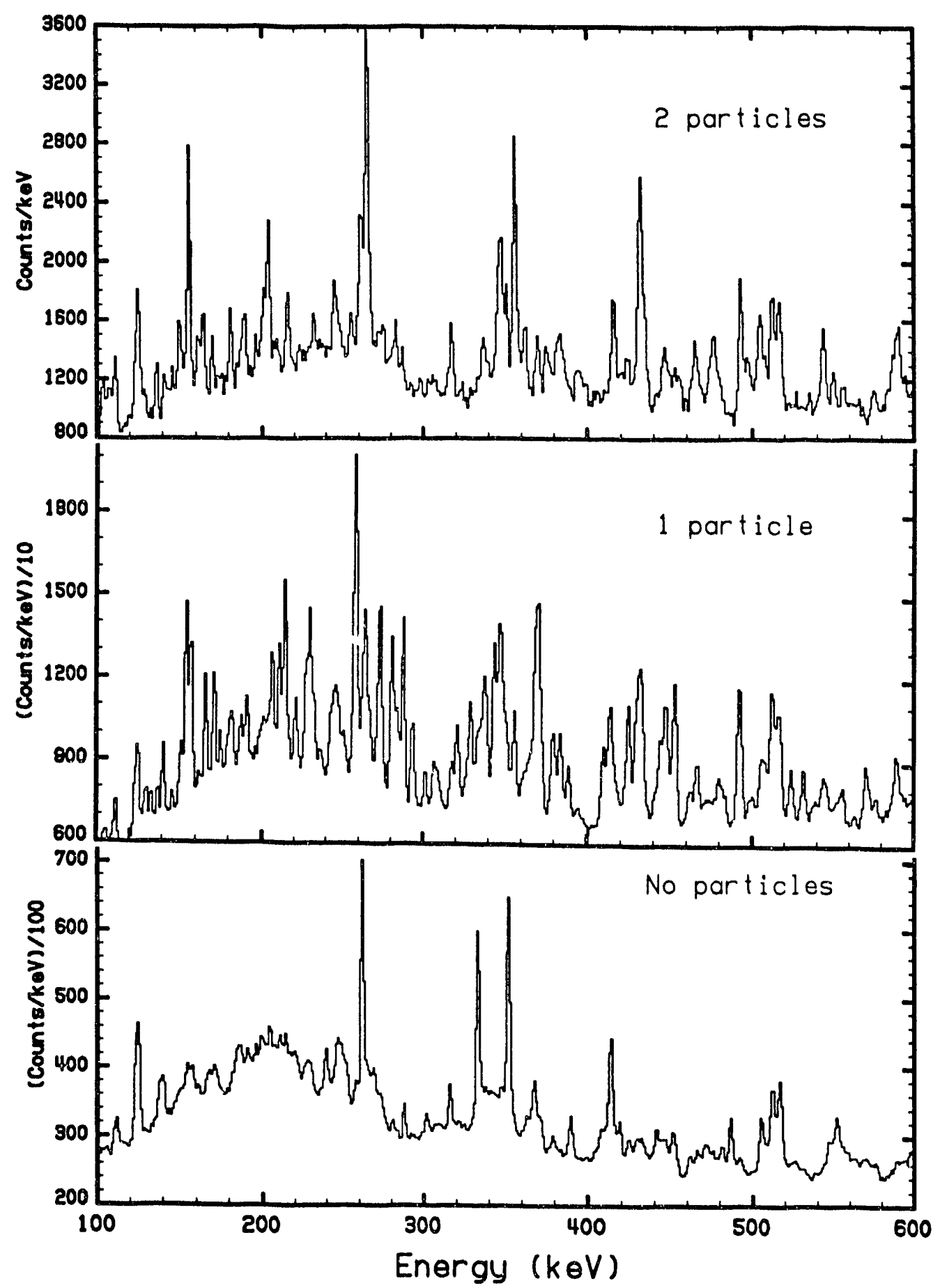

Figure IIIA3.3. Energy spectra of $\gamma$ rays from the reaction ${ }^{32} \mathrm{~S}+{ }^{154} \mathrm{Gd}$ tagged according to the number of paddles that fired. The two paddle spectrum (2-particles) clearly shows $\gamma$ rays from ${ }^{182} \mathrm{Pt}$, the $2 \mathrm{p} 2 \mathrm{n}$ channel. The particle tagged spectra show no contribution from the $\mathrm{xn}$ only channels. The no-particle spectrum shows small contributions from the particles channels which can be subtracted out (see text).

each paddle so that we can place multiplicity and pulse-height gates in the offline analysis. To our surprise, the paddles responded with equal rates to $\gamma$ rays and charged particles. 
This is shown in Fig. IIA3.2, where the low pulse-height peak is due to $\gamma$ rays, while the broader peak is due to charged particles. The average efficiency of each paddle to ${ }^{60} \mathrm{Co} \gamma$ rays was measured to be $1 \%$. Thus, for an average $\gamma$ multiplicity of 18 from the reaction and an average particle multiplicity of 0.7 per event we can explain Fig. IIA3.2.

In this experiment the grazing angle was $88^{\circ}$. Consequently, we had to place sufficient absorber forward of $80^{\circ}$ in order to stop the elastically scattered ${ }^{32} \mathrm{~S}$ particles. This eliminated most of the fission fragment detection. Furthermore, the remaining pulses from the fission fragments overlap with the $\gamma$ peak in the scintillator spectrum. Thus, direct fission fragment counting was not feasible in this experiment. Despite these difficulties, we have evidence that actually the scintillator paddles responded to protons emitted from the excited fission fragments. This is indeed substantial since the compound nucleus ${ }^{186} \mathrm{Hg}$ is very neutron deficient (from statistical model calculations we expect 0.7 protons per fission).

Figure IIIA3.3 shows the total $\gamma$-ray spectra gated on none, one, or two charged particles (paddles firing). The spectra have very little in common, which illustrates the selectivity of the device and its potential for reducing the background. The no-particle spectrum contains about $30 \%$ of the 1-particle spectrum and about $45 \%$ of the 2-particle spectrum statistics. In the offline analysis $30 \%$ and $45 \%$ of the 1- and 2-particle $\gamma \rightarrow \gamma$ matrices, respectively, were subtracted from the no-particle matrix to give a completely charged-particle free and to a considerable degree fission free no-particle $\gamma \rightarrow \gamma$ matrix.

The original idea for this device came from Lee Sobotka. The detector components for this device (scintillator, PMT's, $\mu$-metal shields, and the PMT bases, most of which were spares from the MINIWALL project) came from Sobotka's group. The actual design and construction of the detector, the scattering chamber, target assembly, testing, and implementation was done by our group.

IIIA4. The Mini Wall- R.J. Charity, L.G. Sobotka, G. Van Buren, L. Gallamore, J.T. Hood, J. Elson, and D.G. Sarantites [WU].

As proposed last year we have undertaken and by now completed the construction of a high granularity hodoscope. This device named the Mini Wall was designed to be compatible with the MSU Mini Ball and to complement it because of the Mini Ball's lack of forward granularity which was only comparable to our Dwarf Wall. The Mini Wall, shown in Fig. IIIA2.1 consists of 128 fast plastic - CsI(Tl) phoswich elements. The fast plastic is typically $40 \mu \mathrm{m}$ thick and the $\mathrm{CsI}(\mathrm{T} \ell)$ elements $3.0 \mathrm{~cm}$ thick. The device spans angles from 3.3. - 25.0.

Several changes and advances were made in the operation of the Mini Wall compared to the Dwarf System. The most important is the replacement of the singly gated 16-channel FERA ADC's with the Phillips 7176 individual channel gated ones. This was dictated by the larger distances which result in variations in flight times between any 16 signals. The second is the use of the the new 2-dimensional discriminators, described in some detail in 


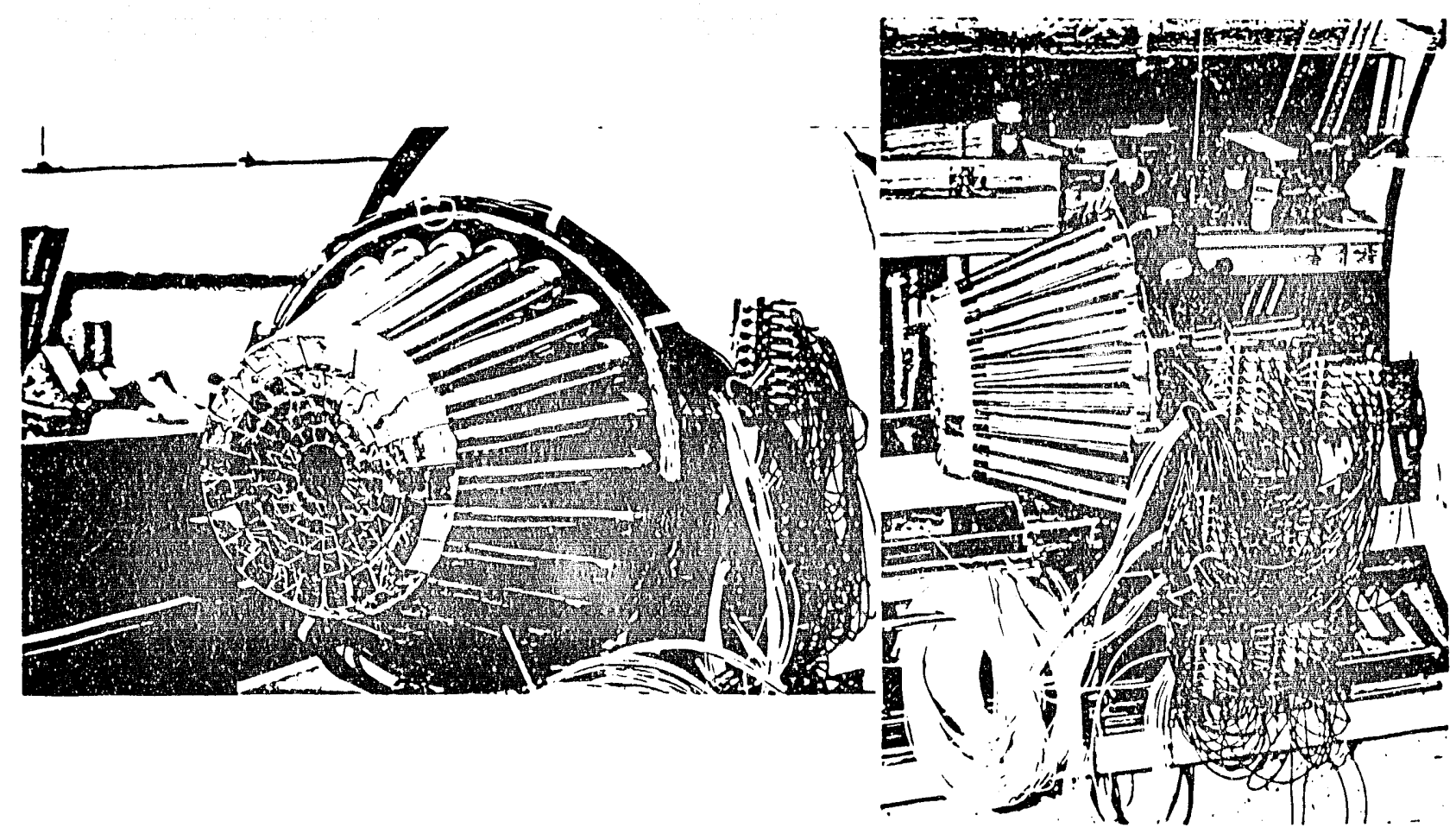

Figure IIIA4.1. Front (left picture) and side view of the WU Miniwall.

the previous section. The last one is the use of the new LeCroy 2277 Multihit TDC's with new features also described in the previous section.

The new detectors of the Mini Wall have superior particle identification capabilities compared to the DBW. They are capable of not only separating $\mathrm{p}, \mathrm{d}, \mathrm{t},{ }^{3} \mathrm{He}$ and He from the intermediate mass fragments, but they also separate ${ }^{6} \mathrm{Li},{ }^{7} \mathrm{Li},{ }^{7} \mathrm{Be}$ and ${ }^{9} \mathrm{Be}$. This is accomplished by use of higher $\mathrm{T} \ell$ doping (1550-1900 ppm) in the $\mathrm{C}_{\mathrm{sI}}\left(\mathrm{T}_{\ell}\right)$ crystals (from Hilger Analytical).

This project was masterminded by L. Sobotka with invaluable assistance from Bob Charity, and with a helping hand from the remaining W.U. nuclear chemistry team. Our group has contributed two major sets of electronic modules, (a) the new 2-dimensional discriminators $(\$ 10,000)$ and (b) the new multihit Lecroy TDC's $(\$ 12,000)$ from funds from this grant. 


\section{IIIB. COMPUTER FACILITY AND DATA ACQUISITION}

\section{IIIB1. The Nuclear Chemistry Computer Facility: Hardware - J. Elson,}

D.G. Sarantites and L.G. Sobotka.

At present we have a local cluster of four 3100 Vax Stations (two 3100 model 76, one model 40, and one model 30). There are five 2.3-Gbyte Exabyte helical tape systems available. The two model 76 work stations have two Exabyte drives each, and the model 40 has one Exabyte and a Cypher 6250/1500 streaming reel tape drive. The output drives include a Tektronix 4596 inkjet plotter and a QMS-810 postscript laser printer. The massive storage capacity of the system includes 3.8 Gibytes on 5 SCSI disk drives.

In order to run commercial graphics packages for publications we acquired an IBM PC compatible (Gateway 2000 - 486 computer). This system was added to the ethernet local cluster as a work station. A second IBM PC/486 serves also as a data acquisition computer. We can either use this in a pulse-height analyzer mode or as a inultidimensional CAMAC based system.

IIIB2. Data Acquisition and Data Analysis Software - J. Elson, and D.G. Sarantites

\section{SNAP: A 1- and 2-dimensional Display and Analysis Program-}

This year we brought to a satisfactory completion a data analysis and display package (1-dim and 2-dim) program called SNAP. This program utilizes the DEC Windows capabilities of the work stations for display and analysis of 2-dimensional and/or 1-dimensional spectral data. It is highly interactive via a mouse or in many instances with quick "Keys". The program is written in $\mathrm{C}$ and has already been exported to the University of Rochester. This program was written by $K$. Myers (supported by this grant).

LZW : A new data compression package -

Recently we completed a new data compression package, that permits us to minimize the disk space required for storing large arrays of histogrammed data and accelerate histogram storage and retrieval from disk.

This package is most valuable in two aspects. Firstly, with the new high resolution arrays such as the miniwall, several hundreds of $2048 \times 2048$ histograms need to be generate 1 and stored for each experiment. Secondly, with the increased capability of the multi-Ge arrays such as Eurogam or Gammasphere multidimensional histograms with (2048) ${ }^{n}$ or $(4096)^{n}$ where $n=3,4$ or 5 are forthcoming. This package can be made to allow storage of such a histogram in 2-Gbyte disks currently available. Details of the performance of this package will be published in a NIM paper.

The new data compression package has several advantages over the old package. Our new format compresses all forms of data. 1-D and 2-D spectra, XY files, and masks. It uses the same data identification fields as the old package, so no user programs need to be changed. 
The old package removed zero data points from 2-D data only, while the new package compresses all data, zero and non-zero alike, using the Lempel-Ziv-Welch algorithm. This algorithm uses a simple method of recognizing patterns of symbols which recur in the data, and reducing the patterns to numbered symbols of varying length. The symbols start out at 9 bits, and can expand up to 16 bits, giving 32768 different symbols. These varying length strings are then packed into 8-bit bytes for storage.

The old package kept the index of spectra at the end of the data, which caused the index to be destroyed whenever a spectrum was added. The updated index was then rewritten after the new spectrum was completely written to the file. This meant that the index did not exist during the writing of a new spectrum. so that if the program, computer, etc. failed to complete writing the spectrum, the entire file's contents were rendered useless. The new package places the index in a reserved space at the beginning of the file, and it is never in an inconsistent state.

The new package achieves a substantial reduction in disk space, so that most files will be between $40-60 \%$ of their former size.

The old package made seven passes through the data when compressing or expanding it, including reserving a big chunk of virtual memory to compress or expand into. If the spectrum does not fit in virtual memory, then the computer needed to page the spectrum in from disk seven times, causing extreme overhead. This has been a severe problem. The new package makes precisely one pass through the data, and reserves no intermediate memory for the compression/decompression, thereby greatly reducing paging activity. The sum of all channels was previously computed every time the data were read (taking up one pass through the data), we now store the sum in the index.

The package has been succesfully tested and its implementation has begun. Shortly it will be the only option of data disk storage in the WU nuclear chemistry group.

An option is provided to allow storage of multiclimensional arrays under predetermined indexed segmentation that will allow easy retrieval of subspaces for the purpose of creating gated or total projections from the matrices.

This package was written by Jon Elson under close consultation with the P.I.

\section{DAMM: The ORNL 2-D display and analysis package:}

We have acquired the ORNL graphics packige DAMM and adopted it to our computer system. It has a number of desirable features that allow convenient gating of a 2-dimensional matrix. Software were written to permit us to write the matrices in the HHIRF format for use with DAMM.

XTERM: A TEK-4010 emulation package:

Recently we acquired a TEK-4010 emulation package (XTERM) that emulates Tektronix graphics on the VMS Workstations. This display interface is extremely fast and allows us to run all our TEK based programs on the DEC windows work stations. 
TEX, LATEX, REVTEX Previewer:

Another acquisition is the previewer for the typesetting packages TEX, LATEX, and REVTEX that runs on the work stations.

IBM PC Analysis and Editing Software:

For the IBM PC's we have acquired the following packages:

1. AXUM: A very versatile graphics package that includes multicurve plotting, contour, surface plots, etc.

2. MATHEMATICA: A symbolic math package with powerful analytical and graphics capabilities.

3. MS WORD 2.0 and Mathtype for Windows.

4. EXCEL for Windows.

5. QUATTRO PRO for DOS.

Acquisition Software:

1. CAMDA : A modified version of a GSI acquisition program that allows easy 1- or 2-dimensional data acquisition via any of the CAMAC modules that we presently own.

2. MAESTRO: The ORTEC data acquisition and analysis program that runs in the MS Windows environment. 


\section{SCIENTIFIC AND TECHNICAL PERSONNEL}

\section{IVA. WASHINGTON UNIVERSIT'Y PERSONNEL}

The following Table summarizes the manpower effort by our group for the period indicated.

\begin{tabular}{llcc}
\hline Person & Position & $\begin{array}{c}\text { Effort During } \\
\text { Last Year (months) }\end{array}$ & $\begin{array}{c}\text { Effort During Last } \\
\text { 3 Years (months) }\end{array}$ \\
\hline D.G. Sarantites & P.I. & 9 & 27 \\
A. Chbihi & Research Associate & - & 12 \\
N.G. Nicolis & Research Associate & 12 & 36 \\
Z. Majka & Visiting Scientist & - & 3 \\
J. Barreto & Visiting Scientist & 12 & 17 \\
R.J. Charity & Research Professor & 1 & 3 \\
V. Abenante & Graduate Student & - & 24 \\
P.F. Hua & Graduate Student & 3 & 3 \\
A. Kirov & Graduate Student & 11 & 11 \\
D. Stracener & Graduate Student & 12 & 36 \\
L. Gallamore & Graduate Student & - & 1 \\
L. Wittmer & Undergraduate & - & 6 \\
K. Myers & Software Specialist & 1 & 10 \\
J. Elson & Electronics Eng. & 8 & 24 \\
J.T. Hood & Design & 3 & 7 \\
\hline & Total (person months) & 72 & 220 \\
& Total (person years) & 6.0 & 18.3 \\
\hline
\end{tabular}

Dan Stracener will be getting his Ph.D. at the end of this semester. Assen Kirov is a graduate student non-candidate for a degree, he will be receiving his $\mathrm{Ph} . \mathrm{D}$. degree from the University of Sofia, Bulgaria, and will be using work done at Washington University as part of his thesis. Peng-Fei Hua is a second year graduate student of our physics department and is doing his thesis work in our group.

We have enjoyed extensive collaboration with Lee Sobotka and his group.

\section{IVB. COLLABORATIONS}

Our group has continued to enjoy fruitful collaborations with numerous members of the Oak Ridge National Laboratory, including J.R Beene, F. Bertrand, C. Baktash, J. Garrett, M.L. Halbert, D.C. Hensley, I.Y. Lee, R. Varner, and N.R. Johnson. 
We have continued our collaboration with C.K. Gelbke, W. Lynch, and M.B. Tsang of MSU.

We have continued our collaboration with the University of Rochester including Udo Schröder and J. Tõke and with M. Riley and his group at the Florida State University. 


\section{PUBLICATIONS}

\section{VA. PAPERS PUBLISHED OR SUBMITTED (1990-1992)}

1. "The Mechanism for the Disassembly of Excited ${ }^{16} \mathrm{O}$ Projectiles into Four Alpha Particles." R.J. Charity, J. Barreto, L.G. Sobotka, D.G. Sarantites, D.W. Stracener, A. Chbihi, and N.G. Nicolis, Phys. Rev. C (in press, Sept. 1992).

2. "Charged Particles as Probes to Study Entrance Channel Effects in the Composite System ${ }^{164}$ Yb." J.L. Barreto, D.G. Sarantites, R.J. Charity, N.G. Nicolis, L.G. Sobotka, D.W. Stracener, D.C. Hensley, J.R. Beene, M. Halbert, and C. Baktash, Proceedings of the Eight Winter Workshop on Nuclear Dynamics, Jackson Hole, Wyomin, Jan. 18-25 (1992), (in press).

3. "EVAP: A Monte Carlo Statistical Model Evaporation Code For High Energy Compound Nucleus Reactions." N.G. Nicolis, D.G. Sarantites, and J.R. Beene. Submitted for publication in NIM (Aug. 1992).

4. "Statistical Emission of Deuterons and Tritons from Highly Excited Compound Nuclei." N.G. Nicolis, D.G. Sarantites, L.G. Sobotka, and R.L. Charity, Phys. Rev. C45 2393 (1992).

5. "Large Deformation in A 170 Nuclei at High Excitation Energies", M. Thoennessen, J.R. Beene, F.E. Bertrand, C. Baktash, M.L. Halbert, D.J. Horen, D.C. Hensley, D.G. Sarantites, D.W. Stracener, W. Spang, and R.L. Varner, Phys. Lett. B282 288 (1992).

6. "Quantitative Studies of Continuum Gamma-ray Correlations in ${ }^{170} \mathrm{Hf}$ " I.Y. Lee, C. Baktash, J.R. Beene, M.L. Halbert, D.C. Hensley, N.R. Johnson, F.K. McGowan, M.A. Riley, and D.G. Sarantites, Phys. Rev. C46 597 (1992).

7. "Binary Character of Highly Dissipative ${ }^{209} \mathrm{Bi}+{ }^{136} \mathrm{Xe}$ Collisions at $\mathrm{E} / \mathrm{A}=28.2 \mathrm{MeV}$ " - B. Lott, S.P. Baldwin, B.M. Szabo, B.M. Quednau, W.U. Schröder, J. Tõke, L.G. Sobotka, J. Barreto, R. Charity, L. Gallamore, D.G. Sarantites, D.W. Stracener, and R.T. de Souza, Phys. Rev, Lett. , 68, 3141 (1992).

8. "Particle Multiplicity Dependence of High-Energy Photon Production in A Heavy-Ion Reaction", L.G. Sobotka, L. Gallamore, A. Chbihi, D.G. Sarantites, D.W. Stracener, W. Bauer, D.R. Bowman, N. Carlin, R.T. deSouza, C.K. Gelbke, W.G. Gong, S. Hannuschke, Y.D. Kim, W.G. Lynch, R. Ronningen, M.B. Tsang, and F. Zhu, J.R. Beene, M.L. Halbert, and M. Thoennessen, Phys. Rev. C44 R2257 (1901)

9. "Yield Decomposition and Excitation Energy Reconstruction in an Incomplete Fusion Reaction", A. Chbihi, L.G. Sobotka, Z. Majka, D.G. Sarantites, D.W. Stracener, V. Abenante, T.M. Semkow, N.G. Nicolis, D.C. Hensley, J.R. Beene and M.L. Halbert, Phys. Rev. C43, 652 (1991). 
10. "Determination of the Nuclear Level Density at High Excitation Energy" A. Chbihi, L.G. Sobotka, Z. Majka, D.G. Sarantites, D.W. Stracener, V. Abenante, T.M. Semkow, N.G. Nicolis, D.C. Hensley, J.R. Beene and M.L. Halbert, Phys. Rev., C43, 666 (1991).

11. "Shape Coexistence and Disappearance of Pairing Correlations in ${ }^{82} \mathrm{Sr} . "$ C. Baktash, G. Garcia-Bermudez, D.G. Sarantites, W. Nazarewicz, V. Abenante, J.R. Beene, H.C. Griffin, M.L. Halbert, D.C. Hensley, N.R. Johnson, I.Y. Lee, F.K. McGowan, M.A. Riley, D.W. Stracener, T.M. Semkow, and A. Virtanen, Phys. Lett B255, 174(1991).

12. "Alpha-Particles as Probes of Nuclear Shape and Structure Effects in Proton Evaporation Spectra", D.G. Sarantites, N.G. Nicolis, V. Abenar te, Z. Majka, T.M. Semkow, C. Baktash, J.R. Beene, G. Garcia-Bermudez, M.L. Halber , D.C. Hensley, N.R. Johnson, I.Y. Lee, F.K. McGowan, M.A. Riley, A. Virtanen and H.C. Griffin, in Proccedings of the 'XXV Zakopane School of Phy: ics' (World Scientific, 1990), Vol.2, selected topics in nuclear structure, Eds. J. Styczen and Z. Stachura, (1990), p.180.

13. "Alpha-Particle Emission as a Probe of Nuclear Shapes and Structure Effects In Proton Evaporation Spectra" N.G. Nicolis, D.G. Sarantites, C. Baktash, V. Abenante, L.A. Adler, J.R. Beene, F.A. Dilmanian, G. Garcia-Bermudez, H.C. Griffin, M.L. Halbert, D.C. Hensley, N.R. Johnson, I.Y. Lee, Z. Majka, F.K. McGowan, M.A. Riley, T.M. Semkow, D.W. Stracener, and A. Virtanen, in Proceedings of the International Conference ' Nuclear Structure in the Nineties ', Oak Ridge, April, 1990, Nucl. Phys. A520, 153c (1990).

14. "Alignment Processes and Shape Variations in ${ }^{184}$ Pt", M.P. Carpenter, C.R. Bingham, L.H. Courtney, V.P. Jansen, A.J. Larabee, Z.-M. Liu, L.L. Riedinger, W.Schmitz [U.T.], R. Bengtsson, T. Bengtsson, W. Nazarewicz, J.-Y. Zhang [JIHIR], J.K. Johansson, D.G. Popescu, J.C. Waddington, [McM], J. Nyberg, A. Johnson, R. Wyss [MSIP], J. Dubuc, G. Kajrys,S. Monaro,S. Pilotte [Montreal], K. Honkanen, D.G. Sarantites [WU], and D.R. Heanni [TAMU], Nucl. Phys. A513, 125 (1990).

15. "Observation of Entrance Channel Effects in Compound Nucleus Formation with High Energy $\gamma$-rays", M. Thoennessen, J.R. Beene, R.L. Auble, F.E. Bertrand, C. Baktash, M.L. Halbert, D.J. Horen, C.A. Ludemann, D.G. Sarantites, D.W. Stracener, and W. Spang, Inst. Phys. Conf. Ser. No 109; Chapter 5 Paper presented at Workshop on Nuclear Structure and Heavy-ion Reaction Dynamics, p.135 (1930).

16. "Dwarf Bali and Dwarf Wall: Design, Instrumentation and Response Characteristics of a $4 \pi \mathrm{CsI}(\mathrm{T} \ell)$ Plastic Phoswich Multidetector System for Light Charged-Particle and Intermediate Mass Fragment Spectrometry", D.W Stracener, D.G. Sarantites, L.G. Sobotka, J. Elson, J.T. Hood, Z. Majka, V. Abenante, A. Chbihi, and D.C. Hensley, Nucl. Instr. Meth. A294, 485 (1990). 
17. "Alpha-Particle and Proton Probes of Nuclear Shapes in the Rare Earth and Mass 80 Regions", D.G. Sarantites, N.G. Nicolis, V. Abenante, Z. Majka, T.M. Semkow, C. Baktash, J.R. Beene, G. Garcia-Bermudez, M.L. Halbert, D.C. Hensley, N.R. Johnson, I.Y. Lee, F.K. McGowan, M.A. Riley, A. Virtanen and H.C. Griffin, Int. Conf. on 'Spectroscopy of Heavy Nuclei 1989' Crete, Greece, 1989. Eds. J. Sharpey Shaffer and L.D. Skouras (World Scientific, 1990), Int. Phys. Conf. Ser. No. 105, (1990) p.39.

18. "Thermal and Angular Momentum Effects in the Giant Dipole Resonance Decay of Hot Rare Earth Nuclei ", H.P. Morsch, W. Spang, J.R. Beene, F.E. Bertrand, R.L. Auble, J.L. Charvet, M.L. Halbert, D.C. Hensley, I.Y. Lee, R.L. Varner, D.G. Sarantites, and D.W. Stracener, Phys. Rev. Lett., 64, 1999 (1990).

19. "Azimouthal Distributions of Fission Fragments and $\alpha$ Particles Emitted in the Reactions ${ }^{36} \mathrm{Ar}+{ }^{238} \mathrm{U}$ at $\mathrm{E} / \mathrm{A}=20$ and $35 \mathrm{MeV}$ and ${ }^{14} \mathrm{~N}+{ }^{238} \mathrm{U}$ at $\mathrm{E} / \mathrm{A}=50$ MeV", M.B. Tsang, Y.D. Kim, N. Carlin, Z. Chen, C.K. Gelbke, W.G. Gong, W.G. Lynch, T. Murakami, T. Nayak, R.M. Ronningen, H.M.Xu, F.Zhu, L.G. Sobotka, D.W. Stracener, D.G. Sarantites Z. Majka, and V. Abenante, Phys. Rev. C42, R15 (1990).

20. "Nuclear Structure Effects in Proton Evaporation Spectra" - D.G. Sarantites, C. Baktash, N.G. Nicolis, G. Garcia-Bermudez, V. Abenante, J.R. Beene, N.R. Johnson, M.L. Halbert, D.C. Hensley, F.K. McGowan, H.C. Griffin, I.Y. Lee, Z. Majka, M.A. Riley, T.M. Semkow, D.W. Stracener, and A. Virtanen, Phys. Rev. Lett., 64, 2129 (1990).

21. "Alpha Particles as Probes of Nuclear Shape in the Rare Earths and Structure Effects on Proton Emission in the Mass 80 Region ", D.G. Sarantites, N.G. Nicolis, V. Abenante, Z. Majka, T.M. Semkow, C. Baktash, J.R. Beene, G. Garcia-Bermudez, M.L. Halbert, D.C. Hensley, N.R. Johnson, I.Y. Lee, F.K. McGowan, M.A. Riley, A. Virtanen, and H.C. Griffin, in The Spectroscopy of Exotic Nuclei, edited by Wm. C. McHarris (Plennum Press, 1990) (in press).

22. "Deformation Effects in the Compound Nucleus Decay Using the Spin Alignment Method", N.G. Nicolis, D.G. Sarantites, L.A. Adler, F.A. Dilmanian, K.J. Honkanen, Z. Majka, L.G. Sobotka, Z. Li, T.M. Semkow, J.R. Beene, M.L. Halbert, D.C. Hensley, J.B. Natowitz, R.P. Schmitt, D. Fabris, G. Nebbia and G. Mouchaty, Phys. Rev. C41, 2118 (1990). 
VB. INVITED PRESENTATIONS IN MEETINGS (1990-1992)

1. "Entrance Channel Effects in Low Energy Fusion Reactions", Invited Talk presented at the ACS Symposium on Nuclear Shapes, Washington D.C. Aug. 23-28 (1992) by D.G. Sarantites, work done with J. Barreto, R.J. Charity, N.G. Nicolis, L.G. Sobotka, D.W. Stracener, J.R. Beene, M.L. Halbert, D.C. Hensley and C. Baktash.

1. "Recent Results with the Dwarf Ball and Wall", D.G. Sarantites, Invited Talk presented at the 'International Conference on Nuclear Physics with Advanved Techniques', Crete, Greece, June (1991).

2. "The Nuclear Level Density of Highly Excited Nuclei", D.G. Sarantites, Invited talk presented at the American Chemical Society Symposium on 'Properties of Nuclei at High Spin', Atlanta, April, (1991).

3. "Incomplete Fusion and the Nuclear Level Density at High Excitations", A. Chbihi, N.G. Nicolis, L.G. Sobotka, D.G. Sarantites, Z. Majka, D.W. Stracener, V. Abenante, T.M. Semkow, D.C. Hensley, J.R. Beene, and M.L. Halbert, Invited Talk presented at the TASCC-90 Workshop, Chalk River, July, 1990.

4. "Alpha-Particle Emission as a Probe of Nuclear Shapes and Structure Effects in Proton Evaporation Spectra", N.G. Nicolis, D.G. Sarantites, C. Baktash, V. Abenante, L.A. Adler, J.R. Beene, F.A. Dilmanian, G. Garcia-Bermudez, H.C. Griffin, M.L. Halbert, D.C. Hensley, N.R. Johnson, I.Y. Lee, Z. Majka, F.K. McGowan, M.A. Riley, T.M. Semkow, D.W. Stracener and A. Virtanen, Invited Talk Presented at the International Conference on 'Nuclear Structure in the Nineties' Oak Ridge, April 23, 1990 (in press).

5. "Alpha-Particles as Probe of Nuclear Shape and Structure Effects in Proton Evaporation Spectra", D.G. Sarantites, N.G. Nicolis, V. Abenante, Z. Majka, T.M. Semkow, C. Baktash, J.R. Beene, G. Garcia-Bermudez, M.L. Halbert, D.C. Hensley, N.R. Johnson, I.Y. Lee F.K. McGowan, M.A. Riley, A. Virtanen and H.C. Griffin, Invited Lecture presented at the 'XXV Zalopane School of Physics', Zakopane, Poland, May 6, 1990 (in press).

6. "Alpha-Particles as Probe of Nuclear in the Rare Earths Structure Effects on Proton Emission in the Mass 80 Region", D.G. Sarantites, N.G. Nicolis, V. Abenante, Z. Majka, T.M. Semkow, C. Baktash, J.R. Beene, G. Garcia-Bermudez, M.L. Halbert, D.C. Hensley, N.R. Johnson, I.Y. Lee F.K. McGowan, M.A. Riley, A. Virtanen and H.C. Griffin, Invited Paper presented at the ACS Symposium on the 'Spectroscopy of Exotic Nuclei', Miami Beach, Florida, September 2, 1989 (in press). 

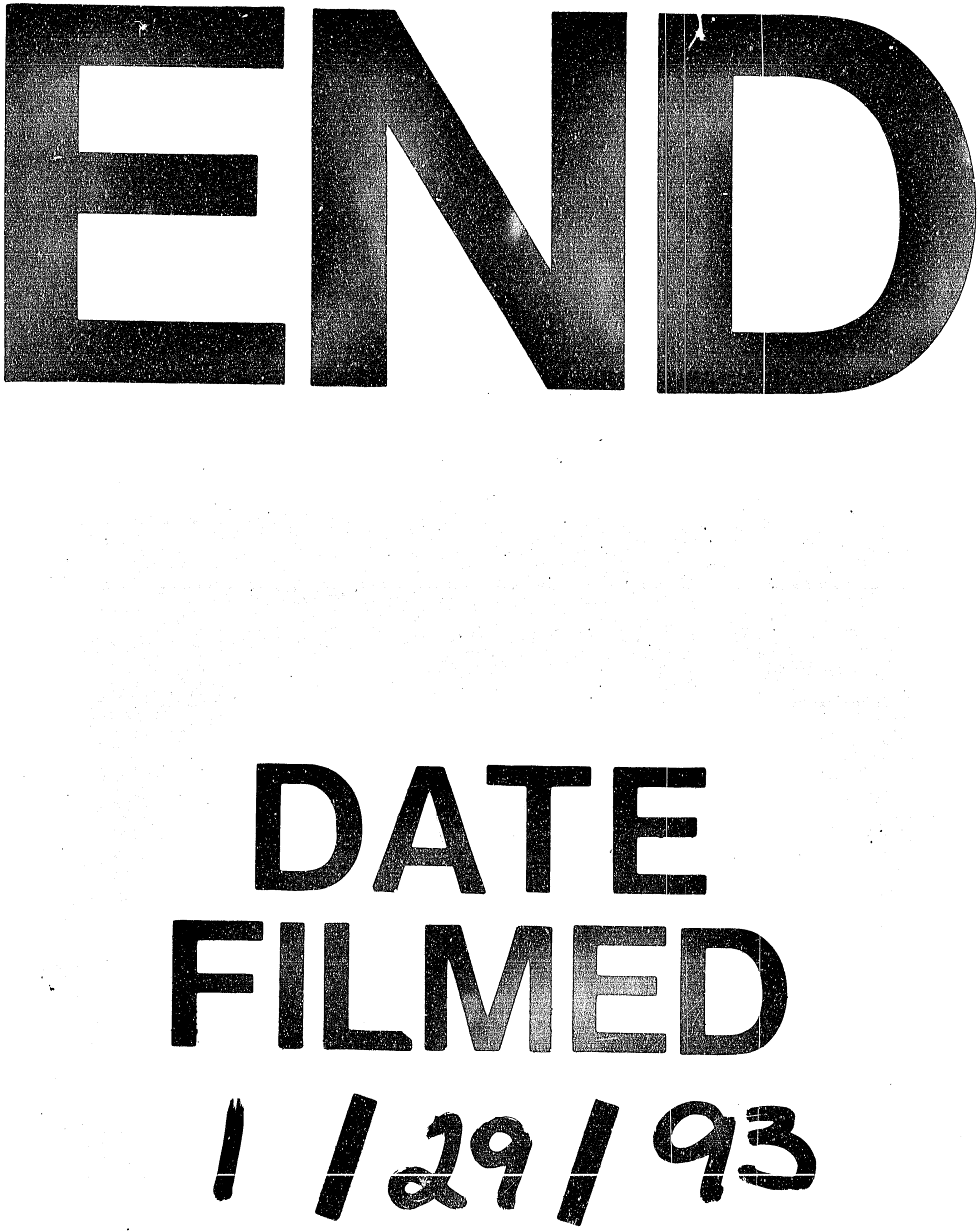
